\title{
SPITZER VIEW OF MASSIVE STAR FORMATION IN THE TIDALLY STRIPPED MAGELLANIC BRIDGE
}

\author{
C.-H. Rosie Chen $^{1,11}$, Remy Indebetouw ${ }^{1,2}$, Erik Muller ${ }^{3,4}$, Akiko Kawamura $^{3,4}$, Karl D. Gordon ${ }^{5}$, Marta Sewieo ${ }^{6}$, \\ Barbara A. Whitney ${ }^{7}$, Yasuo Fukui ${ }^{4}$, Suzanne C. Madden $^{8}$, Marilyn R. Meade ${ }^{7}$, Margaret Meixner $^{5}$, \\ Joana M. Oliveira ${ }^{9}$, Thomas P. Robitaille ${ }^{10}$, Jonathan P. Seale ${ }^{5,6}$, Bernie Shiao ${ }^{5}$, and Jacco Th. Van Loon ${ }^{9}$ \\ ${ }^{1}$ Department of Astronomy, University of Virginia, Charlottesville, VA 22904, USA; rchen@ mpifr-bonn.mpg.de \\ 2 National Radio Astronomy Observatory, Charlottesville, VA 22903, USA \\ ${ }^{3}$ National Astronomical Observatory of Japan, Mitaka, Tokyo 181-8588, Japan \\ ${ }^{4}$ Department of Astrophysics, Nagoya University, Furocho, Chikusaku, Nagoya 464-8602, Japan \\ ${ }^{5}$ Space Telescope Science Institute, Baltimore, MD 21218, USA \\ ${ }^{6}$ Department of Physics and Astronomy, Johns Hopkins University, Baltimore, MD 21218, USA \\ ${ }^{7}$ Department of Astronomy, University of Wisconsin-Madison, Madison, WI 53706, USA \\ ${ }^{8}$ CEA, Laboratoire AIM, Irfu/SAp, Orme des Merisiers, F-91191 Gif-sur-Yvette, France \\ ${ }^{9}$ Astrophysics Group, Lennard-Jones Laboratories, Keele University, Keele, Staffordshire ST5 5BG, UK \\ ${ }^{10}$ Max Planck Institute for Astronomy, D-69117 Heidelberg, Germany \\ Received 2013 September 23; accepted 2014 February 27; published 2014 April 7
}

\begin{abstract}
The Magellanic Bridge is the nearest low-metallicity, tidally stripped environment, offering a unique high-resolution view of physical conditions in merging and forming galaxies. In this paper, we present an analysis of candidate massive young stellar objects (YSOs), i.e., in situ, current massive star formation (MSF) in the Bridge using Spitzer mid-IR and complementary optical and near-IR photometry. While we definitely find YSOs in the Bridge, the most massive are $\sim 10 M_{\odot}, \ll 45 M_{\odot}$ found in the LMC. The intensity of MSF in the Bridge also appears to be decreasing, as the most massive YSOs are less massive than those formed in the past. To investigate environmental effects on MSF, we have compared properties of massive YSOs in the Bridge to those in the LMC. First, YSOs in the Bridge are apparently less embedded than in the LMC: $81 \%$ of Bridge YSOs show optical counterparts, compared to only $56 \%$ of LMC sources with the same range of mass, circumstellar dust mass, and line-of-sight extinction. Circumstellar envelopes are evidently more porous or clumpy in the Bridge's low-metallicity environment. Second, we have used whole samples of YSOs in the LMC and the Bridge to estimate the probability of finding YSOs at a given $\mathrm{H}$ i column density, $N(\mathrm{HI})$. We found that the LMC has $\sim 3 \times$ higher probability than the Bridge for $N(\mathrm{HI})>12 \times 10^{20} \mathrm{~cm}^{-2}$, but the trend reverses at lower $N(\mathrm{HI})$. Investigating whether this lower efficiency relative to $\mathrm{H}$ I is due to less efficient molecular cloud formation or to less efficient cloud collapse, or to both, will require sensitive molecular gas observations.
\end{abstract}

Key words: infrared: stars - Magellanic Clouds - stars: formation - stars: pre-main sequence

Online-only material: color figures, machine-readable tables

\section{INTRODUCTION}

The dependence of star formation on the environment is fundamental in both the nearby and distant universe. Star formation is often enhanced in galaxies undergoing interaction or merger (Larson \& Tinsley 1978). This enhancement can produce global starbursts such as Arp 220, though local concentrations of star forming regions are more frequently observed (Bergvall et al. 2003). Although the overall star formation rate (SFR) can be enhanced, it is not clear how such physical conditions affect the star or cluster mass distribution. The higher pressure and density environment of mergers might encourage the preferential formation of massive stars and clusters (e.g., Elmegreen \& Efremov 1997). On the other hand, the increased turbulence in the interstellar medium (ISM) can result in larger gas dispersions, hampering the formation of giant molecular clouds (GMCs) and hence the massive clusters formed within (e.g., Struck 1997). As massive stars and clusters are the energy source of the ISM and in turn affect the evolution of their host galaxies, it is important to understand their formation in a variety of environments that are different from our Galaxy.

\footnotetext{
${ }^{11}$ Current address: Max Planck Institute for Radio Astronomy, D-53121 Bonn,
} Germany.
In addition to the influence of dynamical interactions, the formation of massive stars may depend on metallicity. A lower dust abundance and greater permittivity to ultraviolet radiation of the ISM is expected to affect pre-formation gas dynamics, as well as cooling and feedback from massive young stellar objects (YSOs; Poglitsch et al. 1995). It is thus critical to understand massive star formation in a low-metallicity, dynamically disturbed environment, to interpret similar situations in the early universe. Furthermore, to understand the detailed physics of the process; the geometric, morphological, and temporal relationships of the molecular and dust components with the forming stellar population, we must spatially resolve the relevant structures, i.e., molecular clouds and individual young massive stars.

Located between the Large Magellanic Cloud (LMC) and the Small Magellanic Cloud (SMC) at a distance of $\sim 50-60 \mathrm{kpc}$ (e.g., Harries et al. 2003; Tabur et al. 2010), the Magellanic Bridge (hereafter the Bridge) is the closest tidal system, and one of the few where clusters and interstellar structures can be resolved and studied in detail. The Bridge was first identified in an H I survey (Hindman et al. 1963), and its production has been suggested to be the result of a recent close encounter between the LMC and SMC 200 Myr ago (e.g., Gardiner et al. 1994; see also Besla et al. 2010). The Bridge's tidal environment together with its significantly low metallicity $\sim 1 / 5-1 / 8 Z_{\odot}$ (Rolleston 


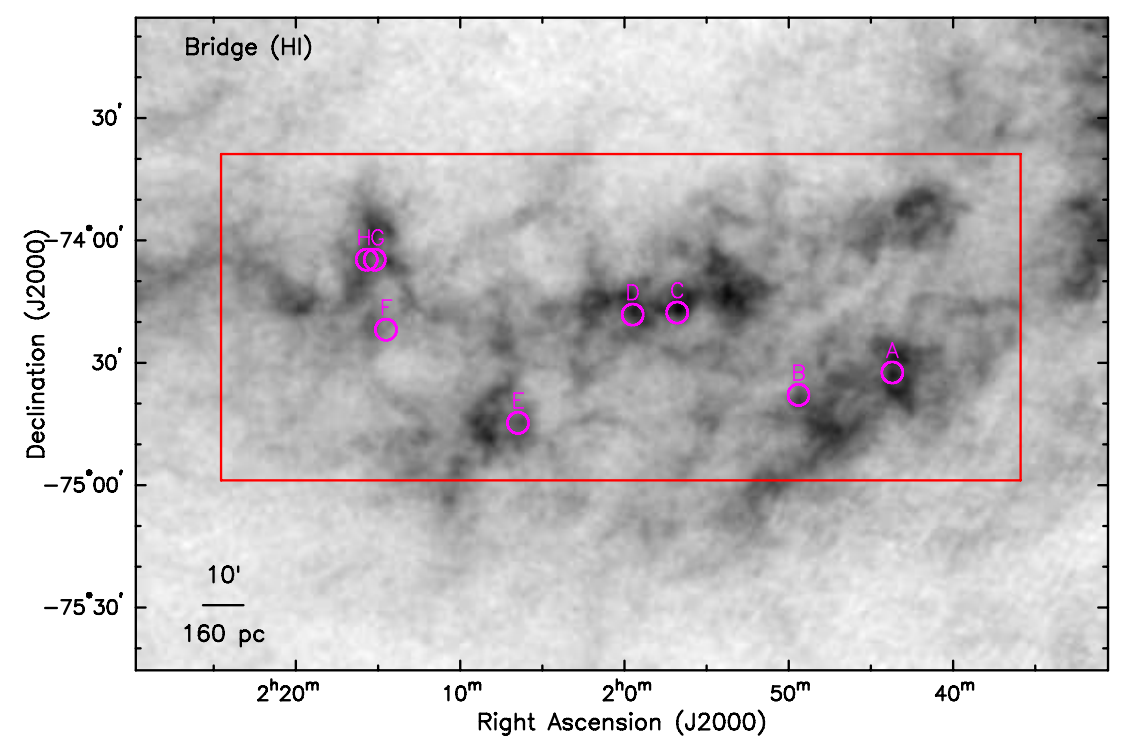

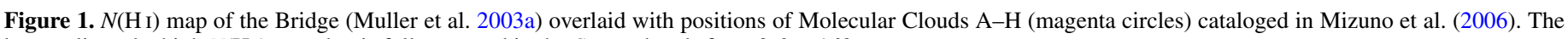
box outlines the high $N(\mathrm{HI})$ area that is fully mapped in the Spitzer bands from 3.6 to $160 \mu \mathrm{m}$.

(A color version of this figure is available in the online journal.)

et al. 1999; Lee et al. 2005) provide an excellent laboratory to study massive star formation under such physical conditions. Furthermore, the Bridge's high $\mathrm{H}_{\mathrm{I}}$ mass $\sim 1.5 \times 10^{8} M_{\odot}$ and substantial H I column density $(N(\mathrm{H} \mathrm{I}))$ up to $\sim 3 \times 10^{21} \mathrm{~cm}^{-2}$ (Muller et al. 2003a) make it a promising site to search for newly formed massive stars. This high H I mass also qualifies the Bridge as a potential region to develop into a dwarf galaxy (H I mass ranging from $10^{6}-10^{9} M_{\odot}$, Mac Low \& Ferrara 1999), providing insight into their development and evolution as well.

Several studies have found evidence of stars in the Bridge less than $\sim 100$ Myr old, which if the 200-300 Myr formation timescale is correct must have formed in situ in tidal gas. Harris (2007) analyzed optical color-magnitude diagrams (CMDs) to derive a star formation history beginning 200-300 Myr ago, with two distinct episodes $\sim 160$ and 40 Myr ago. Studies specifically targeting massive stars provide more compelling evidence of in situ star formation. The handful of large $\mathrm{H} \alpha$ shells and several small H II regions (Meaburn 1986; Muller \& Parker 2007) in the Bridge are most likely formed by massive stars. One of the large $\mathrm{H} \alpha$ shells, DEM S 171, has been suggested to be ionized by one or more O-type stars or blown by a supernova explosion (Meaburn 1986; Graham et al. 2001), though for other $\mathrm{H} \alpha$ shells and regions the underlying stellar population is not known. A population of blue stars are also identified in the Bridge using broadband $B V$ or $B V R$ photometry, with estimated ages ranging from as young as $\sim 10-25 \mathrm{Myr}$ to 100 Myr (Irwin et al. 1990; Demers \& Irwin 1991; Demers \& Battinelli 1998). Nevertheless, sufficient uncertainty in the timescales, and no clear association with natal gaseous material, leave open the possibility that these massive stars actually formed in the SMC body and were stripped out along with the gas. Pre-main-sequence stars identified in the Bridge would require an order of magnitude shorter timescale, and it would be very hard to argue against in situ formation. A recent nearinfrared (NIR) $J H K_{s}$ survey of the Bridge finds Herbig Ae/Be (HAeBe) candidates with ages possibly down to $\sim 2 \mathrm{Myr}$, but this color-selected candidate list is of moderate reliability (only $\sim 40 \%$ likely bona-fide HAeBe; Nishiyama et al. 2007) and requires spectroscopic confirmation.
Recent Spitzer Space Telescope imaging observations in the mid-infrared (MIR) have enabled the detection of individual massive YSOs in the LMC and SMC (e.g., Chu et al. 2005; Whitney et al. 2008; Gruendl \& Chu 2009; Bolatto et al. 2007; Sewiło et al. 2013). Follow-up Spitzer spectroscopic observations further confirms a $>95 \%$ reliability rate in identifying massive YSOs in the LMC using our method based on examination on multi-wavelength spectral energy distributions (SEDs) and images (Chen et al. 2009; Gruendl \& Chu 2009; Seale et al. 2009). In this paper, we present a similar inventory of massive YSOs in the Bridge, compare their properties and distribution to the molecular clouds, and probe a causal relationship between the initial condition (gas) and the end product (stars) in the most direct way. With the knowledge of current and recent stellar content and expected stellar energy feedback, it is then possible to assess if the star formation is triggered and its relative strength to that from spontaneous processes and then to further estimate how the star formation efficiency (SFE) of a molecular cloud varies with time. Comparisons between the SFEs of clouds in the Bridge to those in a variety of metallicities and galactic environments such as the Galaxy or the LMC then allow us to probe the effect of environment on massive star formation.

As part of the Spitzer survey of the SMC (SAGE-SMC; Gordon et al. 2011), the high $N(\mathrm{HI})$ portion of the Bridge $\left(\right.$ where $N(\mathrm{HI})=2-27 \times 10^{20} \mathrm{~cm}^{-2}$ with an average $=10 \times$ $10^{20} \mathrm{~cm}^{-2}$ ) has been mapped fully in the Spitzer bands from 3.6 to $160 \mu \mathrm{m}$ (Figure 1). ${ }^{12}$ Molecular clouds have been detected via CO $J=1-0$ emission (Muller et al. 2003b; Mizuno et al. 2006), providing an excellent opportunity to investigate if the formation of massive YSOs depends on physical conditions of the clouds. To study the current massive star formation in the Bridge, we have used Spitzer MIR observations and archival catalogs and data in the optical and NIR wavelengths. The paper is organized as follows: the observations and data reduction are described

\footnotetext{
12 The region of our study is located in the western part of the continuous stellar bridge between the LMC and SMC (Irwin et al. 1990). It also appears to be extending from the SMC Body and Wing and hence has been referred as "the SMC Tail" (e.g., Gordon et al. 2009). For simplicity we call this region the Bridge.
} 


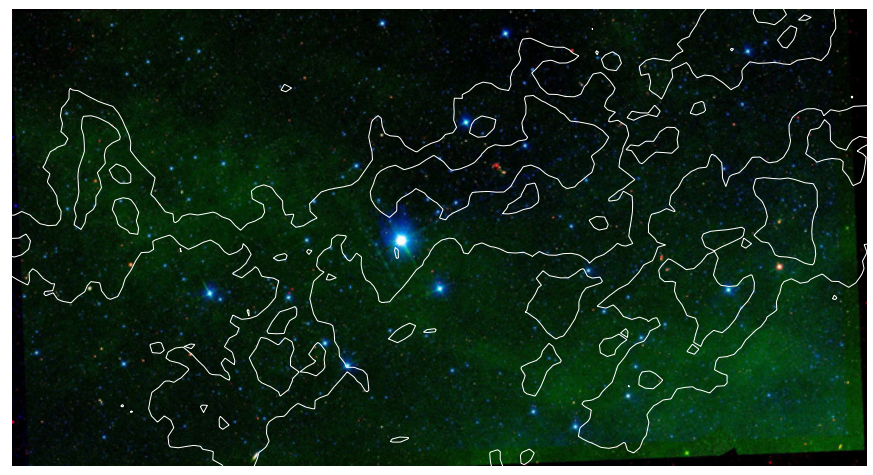

Figure 2. Color composite of the Bridge with Spitzer 3.6, 8.0, and $24 \mu \mathrm{m}$ images mapped in blue, green, and red, respectively. Contours show $N(\mathrm{HI})=11$ and $18 \times 10^{20} \mathrm{~cm}^{-2}$ (40 and $67 \%$ of the peak value, respectively) in white lines. (A color version of this figure is available in the online journal.)

in Section 2; the identification of YSO and HAeBe candidates is reported in Section 3; the derivation of physical properties of these candidates is detailed in Section 4; the properties of massive star formation in the Bridge is discussed in Section 5; and a summary is given in Section 6.

\section{OBSERVATIONS AND DATA REDUCTION}

YSOs are primarily identified by their IR excess, requiring analysis of multiple colors, or equivalently the SED over as wide a wavelength range as possible from optical to IR. Our primary data sets are Spitzer MIR imaging, but to extend the wavelength coverage and improve angular resolution, we include data from several archival ground-based optical and NIR surveys.

\subsection{Spitzer IRAC and MIPS Observations}

The Spitzer observations of the Bridge were obtained as part of the Legacy Program "Surveying the Agents of Galaxy Evolution in the Tidally-Stripped, Low-Metallicity Small Magellanic Cloud" (SAGE-SMC; Gordon et al. 2011). These observations included images taken at 3.6, 4.5, 5.8, and $8.0 \mu \mathrm{m}$ bands with the InfraRed Array Camera (IRAC) and at 24, 70, and $160 \mu \mathrm{m}$ bands with the Multiband Imaging Photometer for Spitzer (MIPS). The details of data processing are given in Gordon et al. (2011). The final mosaics have exposure times $48 \mathrm{~s}$ per pixel at each of the four IRAC bands, and 60, 30, and $\sim 9 \mathrm{~s} \mathrm{pixel}^{-1}$ at MIPS 24,70 , and $160 \mu \mathrm{m}$ bands, respectively. The $180^{\prime} \times 80^{\prime}$ area of the Bridge covered by all IRAC and MIPS bands was analyzed here; it includes all molecular clouds detected in the Bridge. Figure 2 shows the color composite of the analyzed field made with images in the 3.6, 8.0, and $24 \mu \mathrm{m}$ bands, to demonstrate the distribution of different emission. The stellar emission is depicted in the $3.6 \mu \mathrm{m}$ image, the polycyclic aromatic hydrocarbon $(\mathrm{PAH})$ emission is traced in the $8.0 \mu \mathrm{m}$ image, and the dust continuum emission dominates the $24 \mu \mathrm{m}$ image ( $\mathrm{Li} \&$ Draine 2001; Draine \& Li 2007).

The IRAC and MIPS photometry of point sources in the Bridge are available from SAGE-SMC point source catalogs. These catalogs are intended for photometric measurements with consistent quality for point sources, at the expense of excluding sources that are not well fitted by the point-spread functions (PSFs). With IRAC's resolution of $\sim 2$ ", or $\sim 0.6 \mathrm{pc}$ at the Bridge's distance, massive YSOs that have formed compact H II regions or are superimposed on large-scale, diffuse dust features associated with $\mathrm{H}$ II complexes can appear slightly extended or irregular compared to the IRAC PSF and be excluded from the point source catalog. A more complete list can be created by relaxing the point source criteria, but this comes at the expense of significant numbers of unreliable sources (e.g., knots of structured diffuse emission) that must be culled by manual inspection. The process that we used to produce a more complete list is described in detail in Chen et al. (2009, 2010) and is outlined below. Candidate point sources are found with daof ind (Stetson 1987) using relatively inclusive point source criteria. The fluxes of these sources were then measured using the IRAF aperture photometry package apphot with a source aperture of 3 .' 6 (3 pixels) radius and an annular background aperture extending across radii of 3". $6-8$ ". 4 (3-7 pixels), and we applied an aperture correction that was provided in the IRAC Instrument Handbook. ${ }^{13}$ This IRAC catalog is then merged with the SAGE-SMC catalog of MIPS point sources allowing for $1^{\prime \prime}$ positional differences. All of our sources should be unresolved at the poorer spatial resolution of MIPS than IRAC, and hence, they are present in the MIPS point source catalog if they are detected.

\subsection{Additional Data Sets}

To construct multi-wavelength SEDs for sources in the Spitzer catalog, we have expanded it by adding photometry from optical and NIR surveys covering the Bridge, i.e., BRI photometry from the Super COSMOS Sky Surveys (SSS; Hambly et al. 2001b) and $J H K_{s}$ photometry from the Two Micron All Sky Survey (2MASS; Skrutskie et al. 2006). As the photometric limit of the 2MASS catalog is relatively shallow $\left(K_{s} \sim 14.5\right)$ and the long exposure 2MASS catalog, 2MASS 6x, only covers about one third of the area we analyze, the deeper (by $\sim 2$ mag) point source catalog from the InfraRed Survey Facility (IRSF; Kato et al. 2007) is also used to match those IRAC sources without 2MASS counterparts. The data sets are merged by allowing a $1^{\prime \prime}$ position error for matching Spitzer sources with optical or NIR sources.

To examine the large-scale distribution of gas in the Bridge, we have used the $\mathrm{H} \alpha$ images from the Super COSMOS $\mathrm{H} \alpha$ survey (Parker et al. 2005) to examine dense ionized gas and the H I map from the ATCA and Parkes surveys (Muller et al. 2003a) to examine the neutral gas.

\section{IDENTIFICATION OF MASSIVE YSOs}

\subsection{Selection of Massive YSO Candidates}

Owing to the presence of circumstellar dust and hence the excess IR emission, YSOs are positioned in redder parts of CMDs than normal stars without circumstellar dust, such as main-sequence stars. However, redder sources include not only YSOs, but also background galaxies and evolved stars such as asymptotic giant branch (AGB) or post-AGB stars, and these contaminants exist in non-negligible numbers. Figure 3 displays the [8.0] versus ([4.5]-[8.0]) CMD of all sources detected in the Bridge. The prominent vertical branch centered at $([4.5]-[8.0]) \sim 0.0$ is composed mostly of main-sequence, giant, and supergiant stars. Also plotted in Figure 3 are expected loci from models for Galactic C- and O-rich AGB stars (Groenewegen 2006). These loci are only from models for a stellar luminosity of $3000 L_{\odot}$ and can shift vertically from 1.2 to -3.3 mag for the luminosity range $1 \times 10^{3}-6 \times 10^{4} L_{\odot}$ reported for AGB stars (Pottasch 1993).

\footnotetext{
13 http://irsa.ipac.caltech.edu/data/SPITZER/docs/irac/ iracinstrumenthandbook
} 


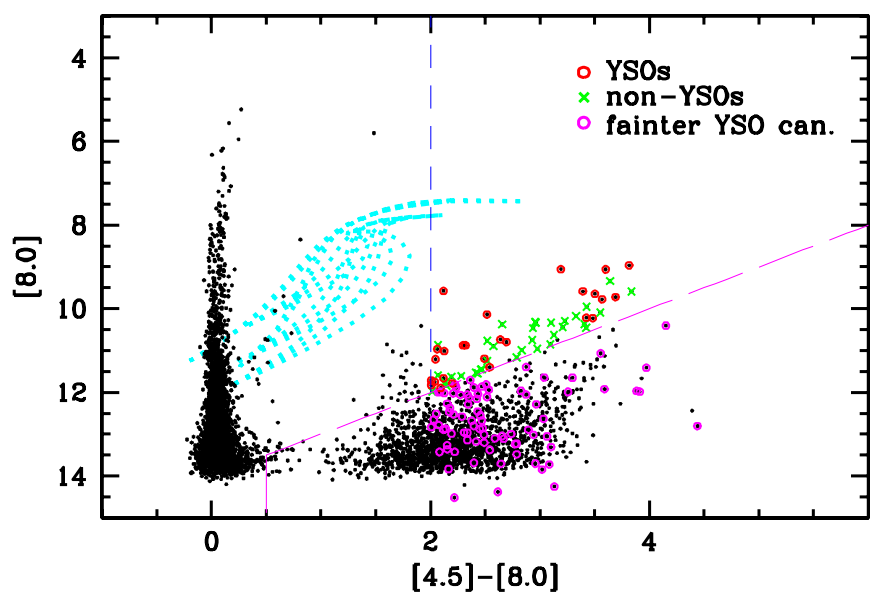

Figure 3. [8.0] vs. [4.5]-[8.0] CMD of all sources detected in the Bridge. Expected loci from AGB stellar models (Groenewegen 2006) are marked with filled cyan squares; only models for a stellar luminosity of $3000 L_{\odot}$ are plotted and hence these loci can shift vertically from 1.2 to -3.3 mag for the luminosity range of AGB stars. The criterion to exclude normal and AGB stars is shown in short-dashed lines and that to exclude background galaxies in long-dashed lines. Sixty YSO candidates are found in the upper right wedge that has the minimum contamination from stars and background galaxies. These candidates have been through detailed examination using multi-wavelength images and SEDs. Candidates that are most likely YSOs are marked with additional red open circles and non-YSOs with green crosses. In addition to these YSOs, the same examination procedures are carried out on 1028 sources in the corresponding lower wedge to identify lower mass or more evolved YSOs. Candidates that are most likely YSOs are marked with additional magenta open circles.

(A color version of this figure is available in the online journal.)

The initial selection of massive YSO candidates was done using two color-magnitude criteria $([4.5]-[8.0]) \geqslant 2.0$ and $[8.0]<14-([4.5]-[8.0])$, which have been demonstrated in Chen et al. (2009) and Gruendl \& Chu (2009) as effective criteria to exclude contaminants such as galaxies and evolved stars. Applying these two criteria to our catalog produces 60 YSO candidates in the Bridge. As a comparison, applying the same criteria to the SAGE-SMC catalog (that have stringent criteria on selecting point sources) produces 25 YSO candidates. Note that the initial list of YSO candidates includes a non-negligible fraction of small dust features, obscured evolved stars, and bright background galaxies that need to be excluded. This requires examining all candidates closely to assess their nature. Following the same procedure outlined in Chen et al. (2009), we examine SSS BRI, 2MASS $J H K_{s}$, and $\mathrm{H} \alpha$ images to better resolve these IR sources and their environments. We also use multi-wavelength SEDs from $B$ band to $70 \mu \mathrm{m}$ constructed from the catalog described in Section 2.2. In the next section, we discuss how we use these images and SEDs to assess whether these candidates are truly YSOs.

\subsubsection{Identification of Contaminants}

Background galaxies, if resolved, can be identified from their morphologies. 17 of the CMD-selected YSO candidates are resolved into galaxies in high-resolution optical BRI and NIR $J H K_{s}$ images, and another 17 candidates show elongated emission extended beyond the point sources, most likely galaxies more distant than the resolved ones. Figure 4 shows $B$-band images and SEDs of two examples from sources in these two categories, a resolved galaxy and a galaxy candidate with extended morphology. The SEDs of the first group of resolved sources with galaxy-like morphology are not well reproduced by YSO models but resemble late-type galaxies, i.e., charac- terized by two broad humps with one over optical and NIR range coming from stellar emission and the other over MIR to FIR range coming from dust emission. The second group of 17 sources have similarly double-peaked SEDs, poorly fit by any of our YSO models. Although we do not have the definitive evidence of spatially resolved imaging and these sources could also be main-sequence stars with nearby dust or more evolved (Class III) YSOs, we classify these additional sources, for a total of 34, as background galaxies.

Unlike YSOs found in the LMC (e.g., Chen et al. 2009; Gruendl \& Chu 2009), background galaxies appear to be the main and only contaminants in the list of CMD-selected YSO candidates. In our LMC studies such lists include a significant number of small dust clumps, local peaks of large-scale dust filaments, and evolved stars. The Bridge does not have bright H II complexes nor the associated photo-dissociation regions (PDRs), and hence, little large-scale diffuse dust emission where dust clumps and peaks are frequently found. The Bridge also has a relatively young stellar population of $\leqslant 200-300$ Myr (Harris 2007, see also Noël et al. 2013 and Bagheri et al. 2013 reporting older stars possibly stripped off from the SMC), and it is thus reasonable that it has few extreme evolved stars (redder than our color selection $([4.5]-[8.0]) \geqslant 2.0)$.

\subsubsection{Massive YSOs and Their Classification}

The results of our examination of 60 YSO candidates are given in Table 1, which includes source name, ranking of the brightness at $8 \mu \mathrm{m}$, magnitudes in the Spitzer bands from 3.6 to $70 \mu \mathrm{m}$, source classification, and remarks. Photometry taken from available optical and NIR catalogs in $B R I J H K_{s}$ bands are listed in Table 2. The identification of YSOs and non-YSOs is shown in the [8.0] versus ([4.5]-[8.0]) CMD in Figure 3. As previously mentioned, among the list of 60 YSO candidates, 34 background galaxies are identified. After excluding these sources, 26 YSO candidates remain. Since these are most likely bona fide YSOs, we will simply call them YSOs in the rest of the paper. Among these 26 YSOs, 20 are from the SAGE-SMC point source catalog and the other 6 are from our IRAC point source catalog produced using more relaxed criteria of the PSFs.

We note that two of the YSOs, J014353.94-743224.71 and J022152.32-744537.83, were previously identified as galaxies LEDA 2816287 and LEDA 248457, respectively, based on their IRAS colors. Their radial velocities, 2199 and $26,087 \mathrm{~km} \mathrm{~s}^{-1}$, respectively, were estimated from $\mathrm{H} \alpha$, [N II], and [S II] lines in low-spatial and low-spectral resolution spectra taken as part of the PSCz redshift survey of IRAS galaxies (Saunders et al. 2000). These velocity estimates are less reliable since these two are not single but are multiple sources. Source J014353.94-743224.71 is resolved into at least five point-like sources of different $(B-R)$ colors within a 4".5-radius circle in high-resolution SSS $B$ and $R$ images at a pixel scale of 0.67 pixel $^{-1}$. These five sources have the clear morphology of a stellar cluster surrounded by fainter stars in the archival $J H K_{s}$ images taken with the ISAAC telescope in the ESO at a superb pixel scale of 0.15 pixel $^{-1}$ (Figure 5). In addition, our Spitzer IRS spectrum of this source shows typical PAH emission features of a YSO. The other source J022152.32-744537.83 is $\leqslant 2$ '.0 from another YSO J022152.99-744534.94 as revealed in all images except at the Spitzer MIPS' poorer resolution. These two neighboring sources have SEDs that are fitted relatively well by YSO models (Figure 6) but quite different from galaxies discussed in Section 3.1.1. Given the above considerations, we thus reclassify 

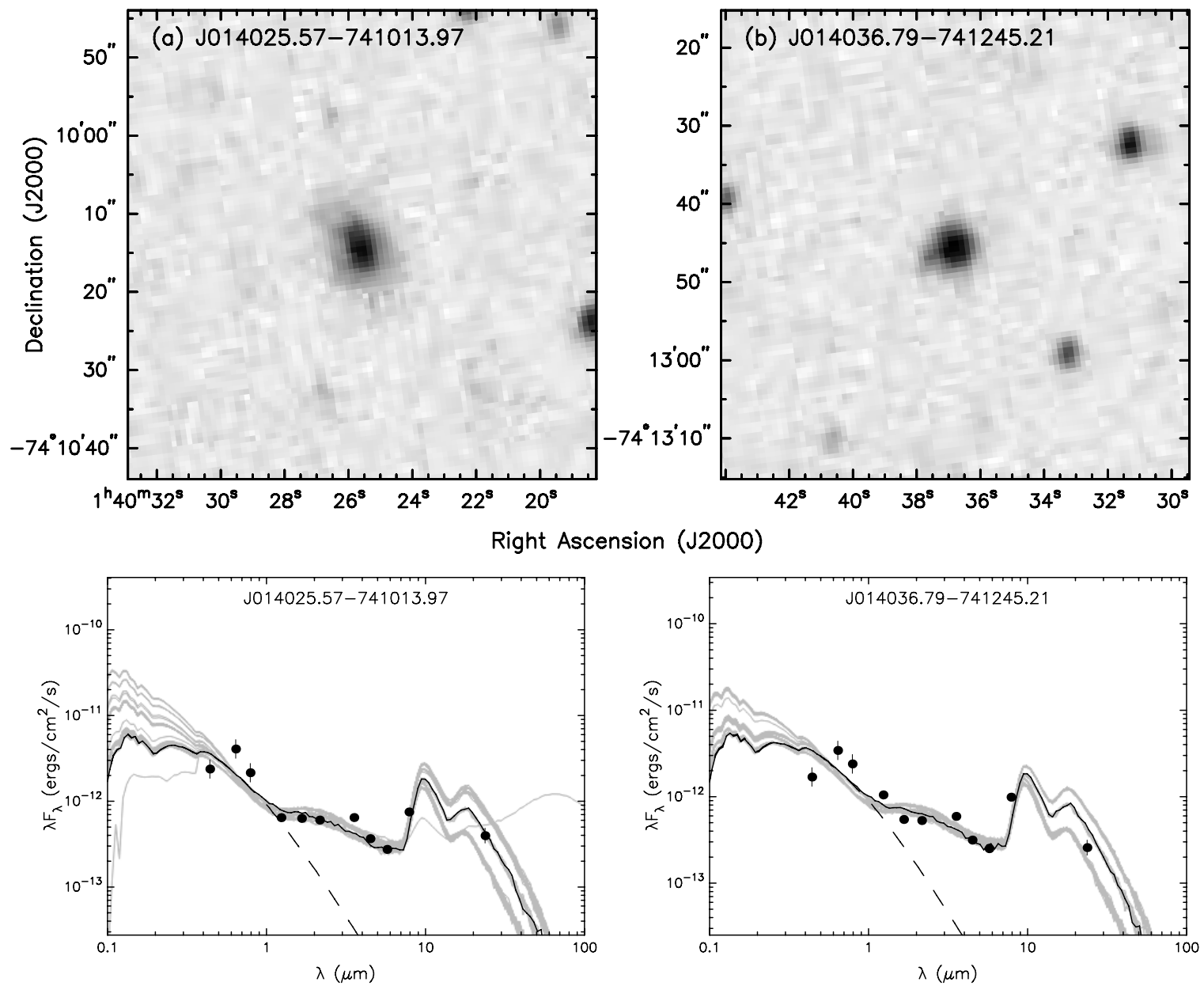

Figure 4. SSS $B$ images and SEDs of two CMD-selected YSO candidates that are most likely galaxies. The observed SEDs of these two sources (filled circles) are not well fitted by YSO models (black and gray lines). Instead, they have SEDs similar to galaxies and are resolved into a spiral galaxy (a) or appear extended (b).

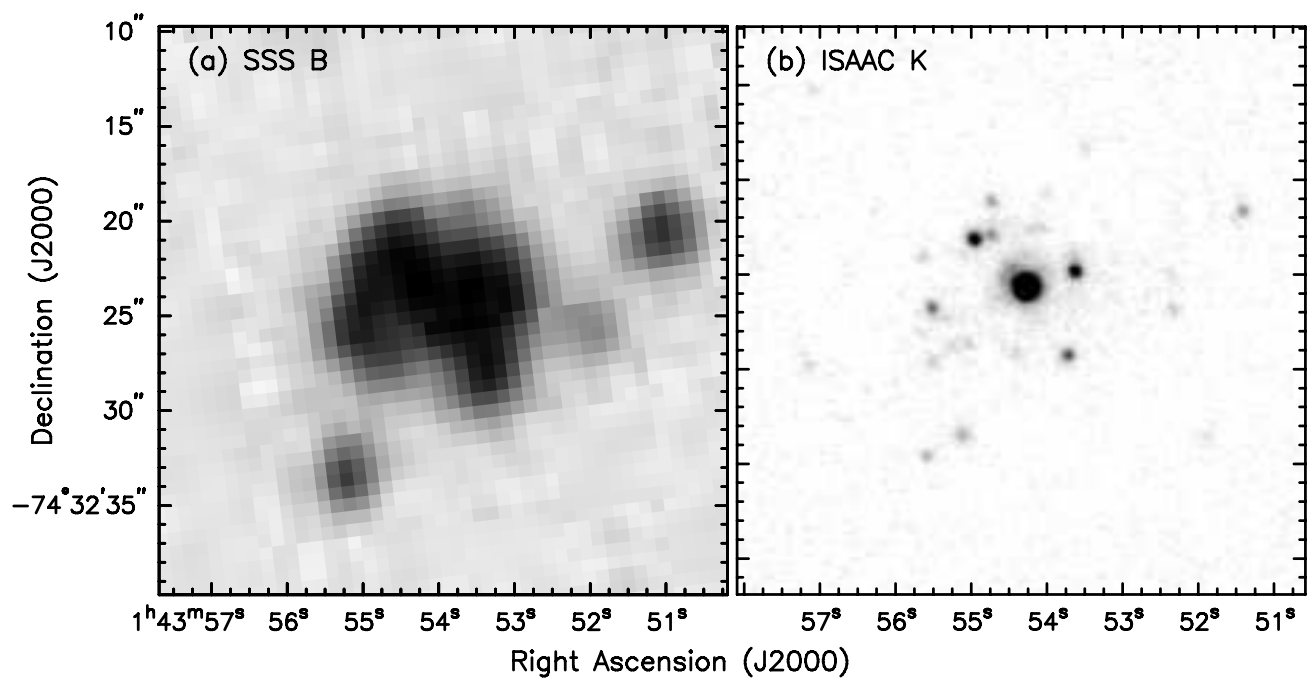

Figure 5. High-resolution SSS $B$ and ISAAC $K$ images of YSO J014353.94-743224.71. This YSO is resolved into a multiple system.

sources J014353.94-743224.71 and J022152.32-744537.83 as YSOs and not as background galaxies.

Using the classification scheme proposed in Chen et al. (2009), we have further categorized the 26 YSOs in the Bridge into Types I, II, and III based on their SEDs and surrounding interstellar environment that are expected as a result of evolution of massive YSOs. Type I YSOs have large circumstellar envelopes that dominate the radiation; thus, their SEDs show a steep rise from the NIR to $24 \mu \mathrm{m}$ and beyond. They are generally not visible at wavelengths shorter than $K_{s}$ band, but they brighten up toward longer wavelengths; they are often found in dark clouds. Type II YSOs show their stellar cores and circumstellar disks after the envelopes have dissipated; thus, their SEDs exhibit a low peak in the optical and a higher peak at 
Table 1

Multi-wavelength Photometry for $\lambda>3 \mu \mathrm{m}$ of YSO Candidates Selected from CMD Criteria

\begin{tabular}{|c|c|c|c|c|c|c|c|c|c|}
\hline $\begin{array}{l}\text { Name } \\
\text { (1) }\end{array}$ & $\begin{array}{l}\text { No. } \\
(2)\end{array}$ & $\begin{array}{c}{[3.6]} \\
(3)\end{array}$ & $\begin{array}{c}{[4.5]} \\
(4)\end{array}$ & $\begin{array}{c}{[5.8]} \\
(5)\end{array}$ & $\begin{array}{c}{[8.0]} \\
(6)\end{array}$ & $\begin{array}{c}{[24]} \\
(7)\end{array}$ & $\begin{array}{c}{[70]} \\
(8)\end{array}$ & $\begin{array}{l}\text { Class. } \\
\text { (9) }\end{array}$ & $\begin{array}{c}\text { Remarks } \\
\text { (10) }\end{array}$ \\
\hline J013928.99-744839.06 & 12 & 13.800 .01 & 13.640 .01 & 12.760 .03 & 10.090 .01 & 6.640 .02 & 1.240 .03 & G & \\
\hline J013958.84-744902.13 & 18 & 13.350 .01 & 13.270 .01 & 12.690 .03 & 10.320 .01 & 7.420 .03 & 2.170 .04 & G & \\
\hline J014025.67-741014.16 & 43 & 13.890 .01 & 13.770 .01 & 13.350 .04 & 11.250 .02 & 8.350 .04 & $\ldots$ & G & \\
\hline J014036.87-741245.39 & 36 & 13.980 .01 & 13.940 .01 & $\ldots$ & 10.960 .01 & 8.820 .06 & $\ldots$ & $\mathrm{G}$ & \\
\hline J014114.46-744823.14 & 38 & 13.850 .01 & 13.820 .02 & 13.260 .04 & 10.990 .01 & 8.060 .04 & 2.600 .05 & $\mathrm{G}$ & \\
\hline J014121.42-734508.53 & 45 & 14.280 .01 & 13.910 .01 & 13.820 .05 & 11.450 .01 & 8.460 .04 & $\ldots$ & $\mathrm{G}$ & \\
\hline J014208.96-735202.92 & 21 & 13.880 .01 & 13.780 .01 & 13.180 .04 & 10.370 .01 & 7.790 .02 & 2.540 .06 & G & \\
\hline J014353.94-743224.71 & 9 & 13.660 .01 & 13.410 .01 & 11.390 .01 & 9.720 .01 & 5.110 .01 & 0.220 .02 & II & mul \\
\hline J014402.46-743333.15 & 47 & 14.350 .01 & 13.940 .01 & 13.730 .06 & 11.530 .02 & 8.730 .05 & $\ldots$ & G & \\
\hline J014536.69-741258.78 & 5 & 12.640 .01 & 11.700 .01 & 10.750 .01 & 9.580 .00 & 5.550 .01 & 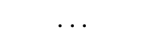 & $\mathrm{I} / \mathrm{II}$ & \\
\hline J014705.47-742601.50 & 44 & 14.930 .01 & 13.940 .01 & 12.710 .02 & 11.400 .01 & 8.060 .03 & & II & \\
\hline J014813.20-734532.97 & 24 & 13.940 .01 & 13.880 .02 & 13.090 .04 & 10.460 .01 & 7.910 .03 & 2.590 .05 & G & \\
\hline J014838.65-744441.71 & 39 & 14.140 .01 & 13.140 .01 & 12.180 .02 & 11.010 .01 & 7.450 .02 & & II/III & \\
\hline J014914.81-733944.20 & 13 & 13.910 .01 & 12.650 .01 & 11.520 .01 & 10.140 .01 & 6.440 .01 & 2.380 .05 & I & \\
\hline J014927.10-740011.41 & 11 & 13.450 .01 & 13.380 .01 & 12.670 .03 & 9.950 .01 & 7.670 .02 & 2.270 .04 & G & \\
\hline J014929.21-743916.48 & 27 & 13.900 .02 & 13.370 .01 & 12.050 .02 & 10.730 .03 & 7.220 .03 & 1.030 .02 & $\mathrm{I} / \mathrm{II}$ & mul \\
\hline J014942.43-743704.76 & 15 & 13.980 .01 & 13.640 .01 & 11.730 .02 & 10.210 .01 & 6.330 .01 & 0.870 .02 & I & mul \\
\hline J015005.67-734714.96 & 25 & 13.680 .01 & 13.400 .01 & 12.570 .02 & 10.460 .01 & 7.280 .02 & $\ldots$ & G & \\
\hline J015039.92-735041.67 & 26 & 13.870 .01 & 13.750 .01 & 13.090 .03 & 10.620 .01 & 7.250 .02 & 2.090 .04 & $\mathrm{G}$ & \\
\hline J015045.05-742337.17 & 41 & 14.650 .01 & 13.690 .01 & 12.570 .02 & 11.200 .01 & 6.990 .02 & 2.100 .04 & $\mathrm{I} / \mathrm{II}$ & \\
\hline J015103.81-745306.07 & 20 & 13.530 .01 & 13.440 .01 & & 10.340 .01 & 7.410 .02 & 2.210 .04 & G & \\
\hline J015113.35-740308.28 & 50 & 14.210 .01 & 13.890 .01 & 13.780 .04 & 11.610 .01 & 8.780 .05 & $\ldots$ & G & \\
\hline J015135.01-735425.52 & 46 & 13.960 .01 & 13.920 .02 & $\ldots$ & 11.450 .01 & 9.510 .15 & $\ldots$ & $\mathrm{G}$ & \\
\hline J015138.67-743000.61 & 23 & 13.870 .01 & 13.650 .01 & 13.170 .03 & 10.440 .01 & 7.260 .02 & 1.990 .04 & $\mathrm{G}$ & \\
\hline J015148.77-745014.92 & 48 & 14.120 .02 & 13.970 .02 & 13.710 .07 & 11.540 .02 & 8.420 .04 & $\ldots$ & G & \\
\hline J015217.87-744755.06 & 58 & 14.220 .01 & 13.980 .02 & & 11.830 .02 & 9.600 .11 & $\ldots$ & G & \\
\hline J015315.03-744510.22 & 33 & & 13.170 .01 & 12.090 .01 & 10.870 .01 & 7.360 .02 & $\ldots$ & II & ext \\
\hline J015435.23-742646.24 & 34 & 14.000 .01 & 13.190 .01 & 12.360 .02 & 10.870 .01 & 7.020 .02 & $\ldots$ & II & \\
\hline J015518.06-743529.29 & 56 & 15.090 .02 & 13.980 .01 & 12.900 .03 & 11.780 .02 & 8.450 .04 & $\ldots$ & II & \\
\hline J015535.26-734110.17 & 49 & 14.100 .01 & 13.660 .01 & 13.640 .05 & 11.590 .01 & 8.180 .03 & $\ldots$ & G & \\
\hline J015717.36-741104.70 & 6 & 13.540 .01 & 13.420 .01 & 12.320 .02 & 9.590 .00 & 7.390 .02 & 1.680 .03 & G & \\
\hline J015809.18-740955.36 & 40 & 14.040 .03 & 13.950 .03 & 13.410 .06 & 11.160 .03 & 8.110 .03 & 2.660 .06 & G & \\
\hline J015857.18-740954.93 & 52 & 14.870 .01 & 13.780 .01 & 12.780 .02 & 11.660 .01 & 8.840 .06 & $\ldots$ & II & \\
\hline J015929.09-742214.05 & 51 & 14.380 .01 & 13.820 .01 & 13.230 .04 & 11.630 .01 & 7.370 .02 & $\ldots$ & G & \\
\hline J020116.73-735926.19 & 14 & 13.670 .01 & 13.500 .01 & $\ldots$ & 10.170 .01 & 7.550 .02 & 1.830 .03 & G & \\
\hline J020159.23-740621.09 & 28 & 13.710 .04 & 13.680 .04 & $\ldots$ & 10.750 .07 & $\ldots$ & 1.570 .02 & $\mathrm{G}$ & \\
\hline J020214.18-741210.61 & 53 & 14.520 .01 & 13.720 .01 & 12.770 .03 & 11.710 .02 & 8.210 .04 & $\ldots$ & $\mathrm{I} / \mathrm{II}$ & ext \\
\hline J020237.42-735549.07 & 29 & 13.290 .01 & 13.280 .01 & 12.840 .03 & 10.770 .01 & 8.610 .05 & $\ldots$ & $\mathrm{G}$ & \\
\hline J020440.85-735746.87 & 55 & 14.280 .01 & 13.900 .01 & 13.820 .06 & 11.760 .02 & 9.100 .07 & $\ldots$ & G & \\
\hline J020503.24-735303.19 & 8 & $\ldots$ & 13.140 .01 & 12.240 .02 & 9.640 .00 & 6.280 .01 & 1.150 .02 & II & ext \\
\hline J020552.13-740445.44 & 54 & 14.780 .01 & 13.790 .01 & 12.860 .03 & 11.750 .02 & 8.700 .05 & $\ldots$ & II/III & \\
\hline J020649.68-744359.95 & 37 & 13.740 .01 & 13.030 .01 & 12.180 .02 & 10.960 .01 & 6.850 .01 & $\ldots$ & II & mul \\
\hline J020744.59-745302.97 & 59 & 14.980 .02 & 13.990 .01 & 13.030 .03 & 11.900 .02 & 8.540 .04 & & II/III & \\
\hline J021246.35-744040.26 & 1 & 12.960 .01 & 12.780 .01 & 11.860 .02 & 8.960 .00 & 5.890 .01 & 0.470 .02 & II & mul \\
\hline J021249.62-740848.22 & 32 & $\ldots$ & 12.940 .01 & 12.540 .03 & 10.870 .02 & 8.580 .05 & 2.460 .05 & G & \\
\hline J021440.18-742127.03 & 30 & 13.940 .03 & 13.490 .03 & 12.410 .05 & 10.790 .05 & 5.260 .01 & -0.720 .01 & I & mul \\
\hline J021505.20-743954.86 & 10 & 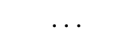 & 13.340 .01 & 12.390 .02 & 9.780 .01 & 6.740 .02 & 1.890 .04 & II & ext \\
\hline J021526.72-740432.73 & 2 & 12.860 .01 & 12.240 .01 & 11.190 .01 & 9.050 .00 & 4.790 .01 & 1.470 .03 & II & ext \\
\hline J021648.92-742412.56 & 22 & 12.980 .03 & 13.030 .03 & 12.120 .06 & 10.370 .07 & 6.130 .01 & 0.610 .02 & G & \\
\hline J021654.37-743558.30 & 4 & $\ldots$ & 12.980 .01 & 11.980 .02 & 9.340 .01 & 6.490 .01 & 1.220 .02 & $\mathrm{G}$ & \\
\hline J021654.69-743940.85 & 60 & $\ldots$ & 14.000 .02 & 13.890 .07 & 11.980 .02 & 8.510 .04 & $\cdots$ & G & \\
\hline J021732.44-744527.82 & 17 & 13.540 .02 & 13.520 .03 & & 10.290 .04 & 8.220 .05 & 1.690 .03 & G & \\
\hline J021822.89-742336.31 & 35 & 13.540 .01 & 13.470 .01 & 13.030 .04 & 10.890 .01 & 8.570 .04 & $\ldots$ & G & \\
\hline J021845.29-734541.11 & 31 & 14.130 .01 & 13.950 .02 & 13.820 .06 & 10.850 .01 & 8.540 .07 & $\ldots$ & $\mathrm{G}$ & \\
\hline J021915.53-745300.02 & 57 & 14.790 .02 & 13.840 .01 & 12.980 .03 & 11.830 .02 & 8.570 .04 & $\ldots$ & II/III & \\
\hline J021936.75-741929.38 & 7 & 12.960 .01 & 12.980 .01 & 11.470 .01 & 9.590 .01 & 6.710 .01 & 1.530 .03 & II & mul \\
\hline J021948.20-743517.55 & 42 & 14.340 .01 & 13.260 .01 & 12.290 .02 & 11.210 .01 & 7.640 .02 & 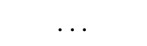 & II & \\
\hline J022030.32-744654.37 & 19 & 13.520 .01 & 13.300 .01 & 13.040 .03 & 10.340 .01 & 7.630 .02 & 2.020 .04 & G & \\
\hline J022152.32-744537.83 & 3 & 12.860 .01 & 12.660 .01 & 11.790 .01 & 9.060 .01 & 5.480 .01 & 0.070 .02 & II & mul \\
\hline J022152.99-744534.94 & 16 & 14.100 .10 & 13.710 .06 & 12.700 .05 & 10.230 .04 & $\ldots$ & $\ldots$ & II & mul \\
\hline
\end{tabular}

Notes. Column 1: source name. Column 2: ranking of the brightness at $8 \mu \mathrm{m}$. Columns 3-8: photometric measurements in 3.6, 4.5, 5.8, 8.0, 24, and 70 $\mu \mathrm{m}$ bands in magnitudes. Measurements with uncertainties of 99.9 are the upper brightness limits as they include fluxes from neighbors or backgrounds. The uncertainties listed here are only errors in measurements and do not include errors in flux calibration, i.e., $5 \%$ in $3.6,4.5,5.8$, and $8.0 \mu \mathrm{m}, 10 \%$ in $24 \mu \mathrm{m}$, and $20 \%$ in $70 \mu \mathrm{m}$. Thus, the total uncertainty of a flux is the quadratic sum of the measurement error and the calibration error. Columns 9 and 10: classification and remarks: ext, extended source; G, background galaxy; I/II/III, Type I/II/III YSO; mul, multiple. 
Table 2

Multi-wavelength Photometry for $\lambda<3 \mu \mathrm{m}$ of YSO Candidates Selected from CMD Criteria

\begin{tabular}{|c|c|c|c|c|c|c|c|c|c|}
\hline $\begin{array}{l}\text { Name } \\
\text { (1) }\end{array}$ & $\begin{array}{l}\text { No. } \\
(2)\end{array}$ & $\begin{array}{c}B \\
(3)\end{array}$ & $\begin{array}{c}R \\
(4)\end{array}$ & $\begin{array}{c}I \\
(5)\end{array}$ & $\begin{array}{c}J \\
(6)\end{array}$ & $\begin{array}{c}H \\
(7)\end{array}$ & $\begin{array}{l}K_{s} \\
(8)\end{array}$ & $\begin{array}{l}\text { Class. } \\
\text { (9) }\end{array}$ & $\begin{array}{c}\text { Remarks } \\
\text { (10) }\end{array}$ \\
\hline J013928.99-744839.06 & 12 & 18.490 .15 & 16.350 .15 & 15.920 .15 & 17.050 .09 & 16.490 .12 & 15.700 .17 & G & \\
\hline J013958.84-744902.13 & 18 & 17.380 .15 & 15.570 .15 & 15.150 .15 & 16.680 .06 & 16.000 .07 & 15.480 .16 & G & \\
\hline J014025.67-741014.16 & 43 & 17.610 .15 & 16.330 .15 & 16.540 .15 & 16.940 .12 & 16.160 .09 & 15.460 .05 & G & \\
\hline J014036.87-741245.39 & 36 & 17.980 .15 & 16.520 .15 & 16.430 .15 & 16.410 .12 & 16.320 .07 & 15.600 .10 & $\mathrm{G}$ & \\
\hline J014114.46-744823.14 & 38 & 17.810 .15 & 16.240 .15 & 16.420 .15 & 16.520 .05 & 15.920 .10 & 15.490 .14 & $\mathrm{G}$ & \\
\hline J014121.42-734508.53 & 45 & 19.160 .15 & 17.370 .15 & 17.050 .15 & $\ldots$ & $\ldots$ & $\ldots$ & $\mathrm{G}$ & \\
\hline J014208.96-735202.92 & 21 & 17.900 .15 & 16.510 .15 & 16.590 .15 & 17.310 .07 & 16.520 .08 & 16.000 .12 & $\mathrm{G}$ & \\
\hline J014353.94-743224.71 & 9 & 13.480 .15 & 15.420 .15 & 15.750 .15 & 16.450 .05 & 16.000 .05 & 15.670 .05 & II & mul \\
\hline J014402.46-743333.15 & 47 & 20.850 .15 & 18.000 .15 & 17.760 .15 & 17.320 .08 & 16.440 .07 & 15.740 .06 & $\mathrm{G}$ & \\
\hline J014536.69-741258.78 & 5 & $\ldots$ & & $\ldots$ & 16.680 .06 & 15.920 .06 & 15.030 .04 & $\mathrm{I} / \mathrm{II}$ & \\
\hline J014705.47-742601.50 & 44 & 19.490 .15 & 18.590 .15 & 18.050 .15 & 17.480 .02 & 16.800 .04 & 16.010 .04 & II & \\
\hline J014813.20-734532.97 & 24 & & & & 17.190 .08 & 16.450 .07 & 16.110 .13 & $\mathrm{G}$ & \\
\hline J014838.65-744441.71 & 39 & 18.310 .15 & 17.830 .15 & 17.400 .15 & 16.550 .02 & 15.910 .02 & 15.810 .06 & $\mathrm{II} / \mathrm{III}$ & \\
\hline J014914.81-733944.20 & 13 & $\ldots$ & 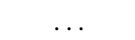 & $\ldots$ & & 17.860 .11 & 16.970 .11 & I & \\
\hline J014927.10-740011.41 & 11 & $\ldots$ & $\ldots$ & $\ldots$ & 16.770 .11 & 15.650 .05 & 15.230 .05 & $\mathrm{G}$ & \\
\hline J014929.21-743916.48 & 27 & $\ldots$ & $\ldots$ & $\ldots$ & 18.050 .21 & 17.190 .18 & 16.260 .10 & $\mathrm{I} / \mathrm{II}$ & mul \\
\hline J014942.43-743704.76 & 15 & $\ldots$ & $\ldots$ & $\ldots$ & & & $\ldots$ & I & mul \\
\hline J015005.67-734714.96 & 25 & 17.480 .15 & 15.880 .15 & 15.480 .15 & 16.160 .13 & 16.230 .14 & 15.880 .21 & G & \\
\hline J015039.92-735041.67 & 26 & 18.260 .15 & 16.690 .15 & 16.650 .15 & $\ldots$ & $\ldots$ & 16.140 .15 & $\mathrm{G}$ & \\
\hline J015045.05-742337.17 & 41 & 21.570 .15 & 19.300 .15 & 100.000 .15 & 18.120 .06 & 17.200 .10 & 16.320 .06 & $\mathrm{I} / \mathrm{II}$ & \\
\hline J015103.81-745306.07 & 20 & $\ldots$ & $\ldots$ & $\ldots$ & 16.760 .05 & 16.170 .06 & 15.630 .11 & G & \\
\hline J015113.35-740308.28 & 50 & 20.090 .15 & 17.830 .15 & 17.340 .15 & 17.300 .15 & 15.890 .07 & 15.520 .14 & $\mathrm{G}$ & \\
\hline J015135.01-735425.52 & 46 & 17.940 .15 & 16.380 .15 & 16.510 .15 & 16.980 .20 & 16.110 .06 & 15.230 .11 & G & \\
\hline J015138.67-743000.61 & 23 & 18.390 .15 & 16.720 .15 & 16.650 .15 & 16.710 .07 & 16.110 .07 & 15.370 .07 & G & \\
\hline J015148.77-745014.92 & 48 & 17.810 .15 & 16.270 .15 & 16.240 .15 & 17.370 .12 & 16.500 .09 & 15.750 .08 & G & \\
\hline J015217.87-744755.06 & 58 & $\ldots$ & $\ldots$ & $\ldots$ & 17.360 .06 & 16.540 .11 & 15.750 .08 & G & \\
\hline J015315.03-744510.22 & 33 & 19.860 .15 & 18.600 .15 & 17.800 .15 & 16.790 .02 & 16.190 .02 & 15.470 .03 & II & ext \\
\hline J015435.23-742646.24 & 34 & 18.480 .15 & 17.520 .15 & 17.210 .15 & 17.140 .04 & 16.610 .05 & 15.950 .05 & II & \\
\hline J015518.06-743529.29 & 56 & 20.500 .15 & 18.850 .15 & 100.000 .15 & & 17.720 .08 & 16.920 .09 & II & \\
\hline J015535.26-734110.17 & 49 & 19.150 .15 & 17.080 .15 & 17.070 .15 & 17.650 .10 & 16.160 .10 & 15.560 .08 & G & \\
\hline J015717.36-741104.70 & 6 & 19.000 .15 & 17.300 .15 & 17.190 .15 & 16.840 .07 & 16.020 .06 & 15.350 .10 & G & \\
\hline J015809.18-740955.36 & 40 & $\ldots$ & $\ldots$ & $\ldots$ & 16.970 .05 & 16.430 .07 & 15.700 .11 & $\mathrm{G}$ & \\
\hline J015857.18-740954.93 & 52 & $\ldots$ & $\ldots$ & $\ldots$ & $\ldots$ & $\ldots$ & $\ldots$ & II & \\
\hline J015929.09-742214.05 & 51 & 21.160 .15 & 18.290 .15 & 18.100 .15 & 17.230 .07 & 16.840 .15 & 15.880 .09 & G & \\
\hline J020116.73-735926.19 & 14 & & & $\ldots$ & 17.110 .07 & 16.160 .07 & 15.740 .12 & G & \\
\hline J020159.23-740621.09 & 28 & 15.360 .15 & 14.660 .15 & 14.880 .15 & 16.630 .04 & 16.120 .06 & 15.770 .12 & $\mathrm{G}$ & \\
\hline J020214.18-741210.61 & 53 & $\ldots$ & $\ldots$ & $\ldots$ & 18.380 .05 & 17.470 .07 & 16.790 .10 & $\mathrm{I} / \mathrm{II}$ & ext \\
\hline J020237.42-735549.07 & 29 & 17.390 .15 & 15.870 .15 & 15.750 .15 & 15.730 .02 & 15.550 .05 & 14.850 .05 & G & \\
\hline J020440.85-735746.87 & 55 & 19.560 .15 & 17.450 .15 & 17.230 .15 & 16.870 .09 & 15.890 .05 & 15.070 .05 & G & \\
\hline J020503.24-735303.19 & 8 & 18.590 .15 & 16.770 .15 & 16.620 .15 & 16.250 .03 & 14.820 .04 & 14.860 .09 & II & ext \\
\hline J020552.13-740445.44 & 54 & 19.380 .15 & 18.320 .15 & 18.220 .15 & 17.660 .04 & 17.180 .06 & 16.660 .07 & $\mathrm{II} / \mathrm{III}$ & \\
\hline J020649.68-744359.95 & 37 & 19.210 .15 & 17.800 .15 & 18.640 .15 & 17.790 .05 & 16.910 .04 & 15.810 .04 & II & mul \\
\hline J020744.59-745302.97 & 59 & 19.210 .15 & 18.310 .15 & 17.930 .15 & 17.430 .02 & 17.080 .04 & 16.670 .06 & II/III & \\
\hline J021246.35-744040.26 & 1 & 17.220 .15 & 16.090 .15 & 16.020 .15 & & 16.110 .05 & 15.680 .15 & II & mul \\
\hline J021249.62-740848.22 & 32 & 16.820 .15 & 15.410 .15 & 15.260 .15 & 15.900 .05 & 15.210 .04 & 14.690 .06 & G & \\
\hline J021440.18-742127.03 & 30 & & & & 15.470 .02 & 15.430 .03 & 15.370 .04 & I & mul \\
\hline J021505.20-743954.86 & 10 & 17.940 .15 & 16.420 .15 & 16.200 .15 & 16.660 .05 & 15.880 .06 & 15.550 .10 & II & ext \\
\hline J021526.72-740432.73 & 2 & $\ldots$ & $\ldots$ & $\ldots$ & 15.670 .02 & 15.190 .02 & 14.540 .03 & II & ext \\
\hline J021648.92-742412.56 & 22 & 10.620 .15 & 13.720 .15 & 13.960 .15 & 15.850 .06 & 15.350 .12 & 14.680 .05 & G & \\
\hline J021654.37-743558.30 & 4 & 16.850 .15 & 15.650 .15 & 15.600 .15 & 16.450 .05 & 15.580 .05 & 15.230 .05 & G & \\
\hline J021654.69-743940.85 & 60 & & & & 17.310 .06 & 16.600 .05 & 16.060 .12 & G & \\
\hline J021732.44-744527.82 & 17 & 13.360 .15 & 15.180 .15 & 15.300 .15 & 16.700 .06 & 16.250 .08 & 15.990 .14 & G & \\
\hline J021822.89-742336.31 & 35 & 17.950 .15 & 16.380 .15 & 16.280 .15 & 16.920 .06 & 15.840 .16 & 15.070 .10 & G & \\
\hline J021845.29-734541.11 & 31 & 18.170 .15 & 16.800 .15 & 16.400 .15 & 17.250 .05 & 16.720 .10 & 16.340 .14 & $\mathrm{G}$ & \\
\hline J021915.53-745300.02 & 57 & 18.870 .15 & 18.350 .15 & 17.960 .15 & 17.690 .04 & 16.980 .05 & 16.590 .06 & $\mathrm{II} / \mathrm{III}$ & \\
\hline J021936.75-741929.38 & 7 & 15.760 .15 & 15.170 .15 & 15.360 .15 & 16.510 .05 & 15.720 .06 & 15.390 .09 & II & mul \\
\hline J021948.20-743517.55 & 42 & $\ldots$ & $\ldots$ & $\ldots$ & 17.060 .03 & 16.530 .05 & 16.100 .09 & II & \\
\hline J022030.32-744654.37 & 19 & 18.550 .15 & 16.720 .15 & 16.550 .15 & & & 15.110 .08 & G & \\
\hline J022152.32-744537.83 & 3 & $\ldots$ & $\ldots$ & $\ldots$ & 15.760 .03 & 15.110 .03 & 15.210 .14 & II & mul \\
\hline J022152.99-744534.94 & 16 & $\ldots$ & $\ldots$ & $\ldots$ & 16.520 .05 & 15.440 .07 & 15.730 .18 & II & mul \\
\hline
\end{tabular}

Notes. Column 1: source name. Column 2: ranking of the brightness at $8 \mu \mathrm{m}$. Columns 3-8: BRIJHK photometric measurements in magnitudes. Measurements with uncertainties of 99.9 are the upper brightness limits as they include fluxes from neighbors or backgrounds. The uncertainties listed here are only errors in measurements and do not include errors in flux calibration, i.e., $10 \%$ in $B R I J H K_{s}$. Thus, the total uncertainty of a flux is the quadratic sum of the measurement error and the calibration error. Columns 10 and 11: classification and remarks: ext, extended source; G, background galaxy; I/II/III, Type I/II/III YSO; mul, multiple. 

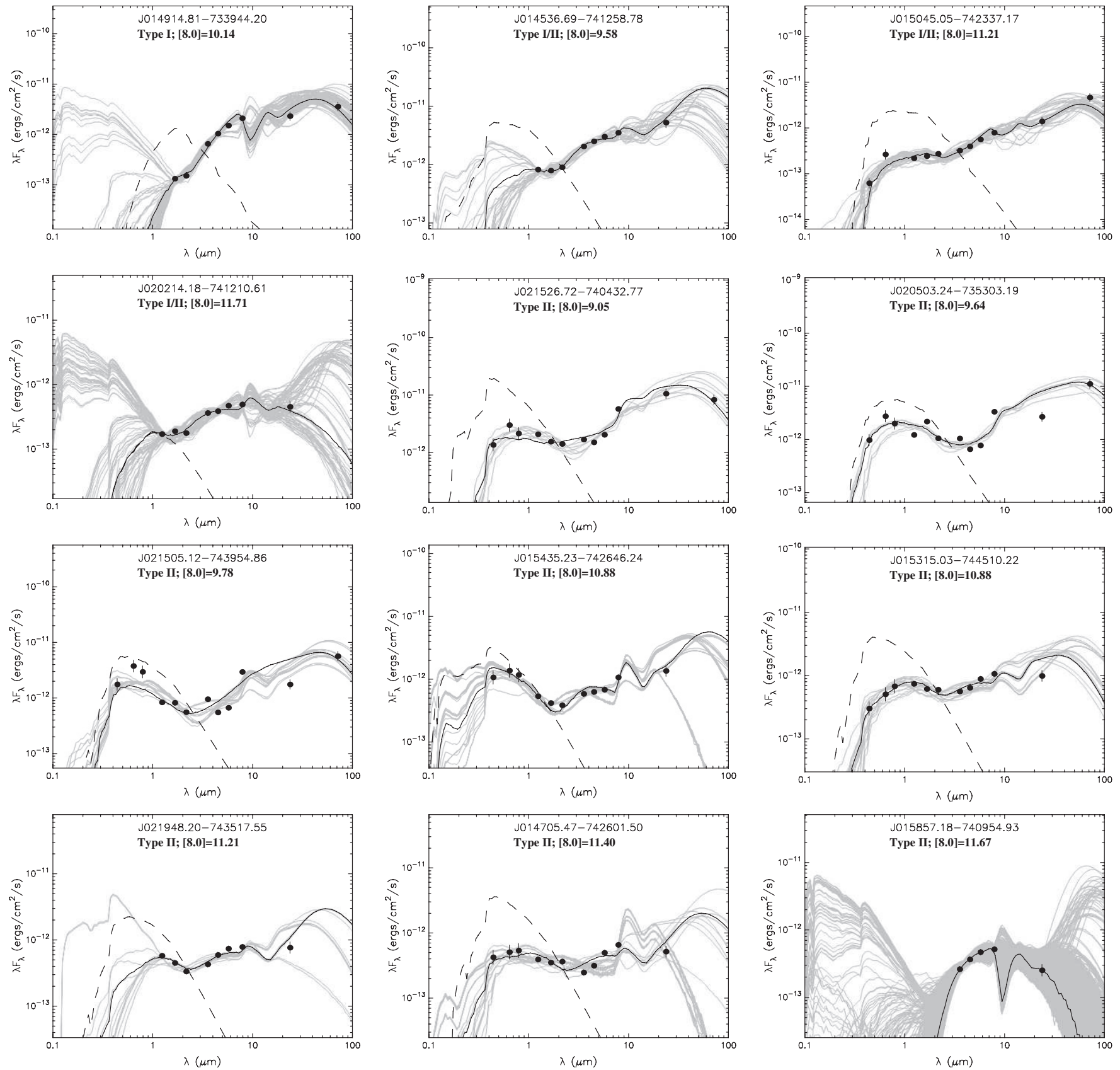

Figure 6. SEDs of 26 YSOs identified in the Bridge. Filled circles are the flux values converted from magnitudes listed in Tables 1 and 2 . The source names are labeled at the top of the plot. Triangles are upper limits. Error bars are shown if larger than the data points. The solid black line shows the best-fit model, and the dashed black line illustrates the radiation from the central star reddened by the best-fit foreground extinction $A_{V}$. The gray lines show all acceptable models.

$8-24 \mu \mathrm{m}$. They are faint in the optical but bright in the near- to MIR up to $8 \mu \mathrm{m}$, and then fading at $24 \mu \mathrm{m}$. Type III YSOs have largely exposed their stellar cores and possessed only remnant circumstellar material; thus, their SEDs peak in the optical and show only modest dust emission in the NIR to MIR. They are bright in the optical and fading at longer wavelengths; they are often associated with small $\mathrm{H}$ II regions.

This "Type" classification is straightforward for YSOs that are unresolved by IRAC but is more complicated for those in multiple systems or complex surroundings. Nine of the twentysix YSOs are resolved by the SSS and 2MASS images into multiple sources within the IRAC PSF (e.g., the aforementioned YSO J014353.94-743224.71), and another five appear more extended than the PSF of SSS and 2MASS that need higher- resolution images such as the on-going VISTA survey of the Magellanic Clouds System (VMC; Cioni et al. 2011) to identify multiple components. YSOs are often found in dark clouds, dust columns, or $\mathrm{H}$ II regions. These interstellar features can be identified in high-resolution optical images, but the MIR emission from these dust features can be blended with that of the YSOs in the Spitzer images, especially in the $24 \mu \mathrm{m}$ band at a resolution $\sim 6^{\prime \prime}$, increasing the uncertainty of the YSO's classification.

Our classification of the 26 YSOs and remarks on the multiplicity and association with the dark cloud, dust column, and $\mathrm{H}_{\mathrm{II}}$ region are given in Table 1. For YSOs in multiple systems or complex interstellar surroundings, the classification has larger uncertainties. 

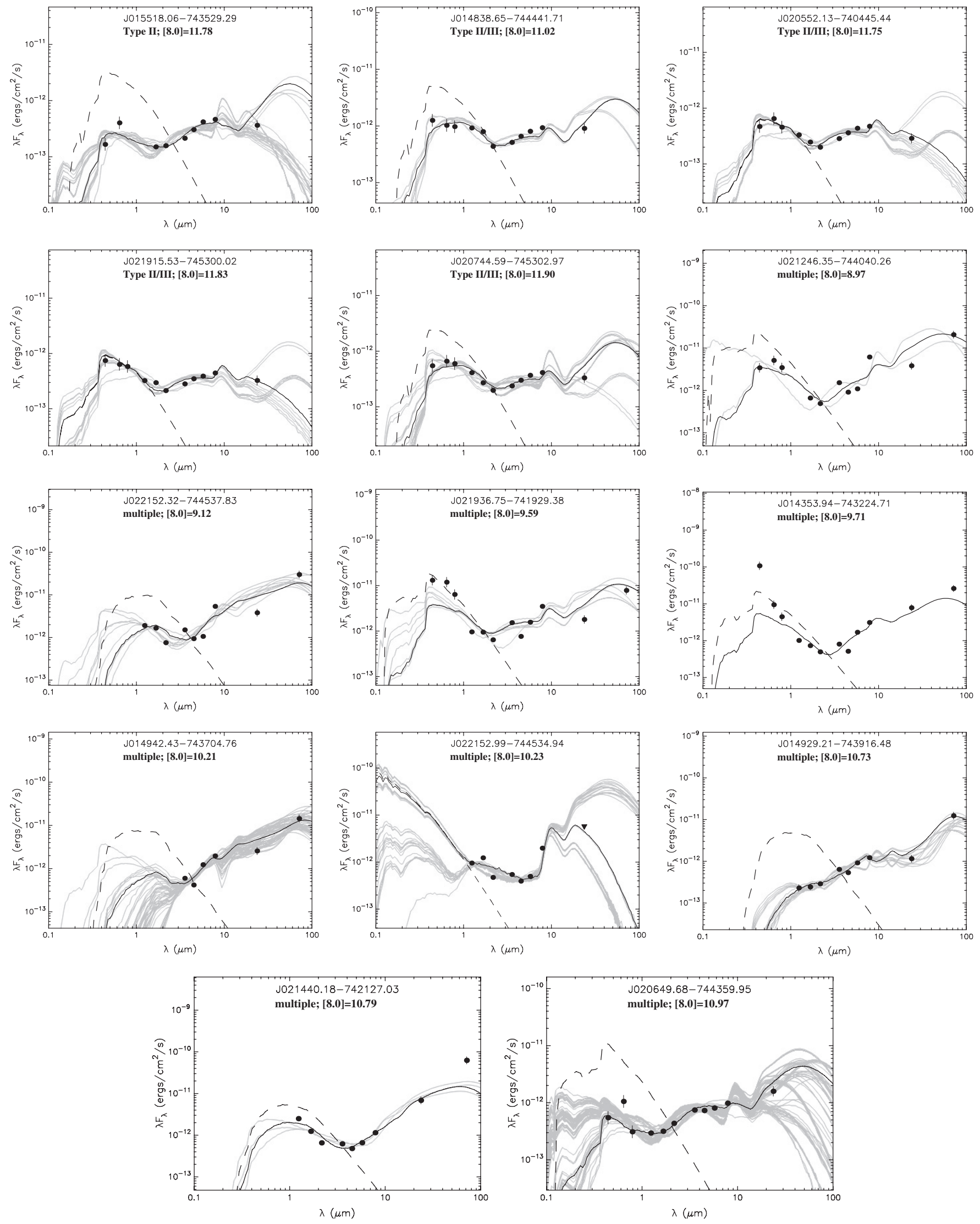

Figure 6. (Continued) 


\subsection{Selection of Fainter YSO Candidates}

The lower part of the [8.0] versus ([4.5]-[8.0]) CMD (Figure 3 ) is populated by numerous background galaxies (Harvey et al. 2006) as well as YSOs that have masses lower than $\sim 4 M_{\odot}$, and more evolved YSOs with reduced circumstellar dust and infrared excess (Whitney et al. 2004a; Robitaille et al. 2006). The primary selection above excludes these YSOs in order to reduce the number of background galaxies.

Among the eight molecular clouds detected in the Bridge (Mizuno et al. 2006), the most massive one, Molecular Cloud $\mathrm{C}$ does not have sources that meet our initial YSO selection criteria, but a cluster of eight red sources fainter than the color-magnitude cut bounded by [8.0] $\geqslant 14-([4.5]-[8.0])$. To assess the nature of these sources, we examined their multiwavelength SEDs and images. One of them was resolved into a spiral galaxy in the archival ISAAC images, and another has a galaxy-like SED as discussed in Section 3.1.1; these two are most likely background galaxies. The remaining six red sources have SEDs similar to YSOs. Furthermore, our IRS spectra of the two brightest $24 \mu \mathrm{m}$ sources among them show PAH features or red continuum with a rising slope, typical of YSOs (R. Indebetouw et al., in preparation). Given the above considerations and that these six sources are in a molecular cloud, they are most likely YSOs.

We have further expanded the examination of SEDs and images on all sources within the same lower wedge in the CMD, to assess if they are YSOs. Among 1028 sources found in this wedge, 192 have photometric measurements less than four bands to effectively distinguish their nature; 753 are resolved into galaxies in high-resolution SSS or 2MASS images, or have SEDs that are not well reproduced by YSO models but resemble late-type galaxies, or active galactic nuclei, i.e., flat from optical to FIR or obscured in the optical due to the viewing angle with respect to its dust torus (Franceschini et al. 2005; Hatziminaoglou et al. 2005; Rowan-Robinson et al. 2005). Furthermore, resolved galaxies with similar SEDs are found in close vicinities of most of these 753 sources, suggesting that they are most likely bona fide galaxies. After excluding the above 192 and 753 sources, 83 sources remain. These sources are likely YSO candidates, though a fraction of them could be star-forming (dwarf) galaxies having SEDs similar to YSOs but unresolved in the SSS or 2MASS images. Note that such confusion is much less serious in our primary CMD-selected YSOs because galaxies would have to be quite nearby to be that bright and hence are usually resolved in the SSS or 2MASS images. Given that these 83 fainter sources contain a fraction of contaminants and to distinguish them from the primary highconfidence sample, we will call them fainter YSO candidates in the rest of the paper. The results of identifying these fainter YSO candidates are shown along with the primary YSOs in the [8.0] versus ([4.5]-[8.0]) CMD (Figure 3).

Finally, we recall that our both sets of selection criteria excluded YSOs with [4.5]-[8.0] $<2.0$, i.e., those that have much less circumstellar dust owing to even lower $M_{\star}$ or later evolutionary stages than their redder counterparts. Identifying YSOs in this bluer part of the CMD is more challenging as the contamination from evolved stars such as AGB or post-mainsequence Be stars is significant (Boyer et al. 2011; Bonanos et al. 2010), and often requires additional data such as light curves or spectral lines to distinguish YSOs from these contaminants (e.g., de Wit et al. 2003, 2005). Sewiło et al. (2013) proposed a statistical approach to select YSO candidates in the SMC from five sets of CMD criteria that include sources outside our selection criteria. They calculated each source's location in the color-magnitude space with respect to contaminants, determined a "CMD score" as a measure of confidence level for this source being a non-contaminant, and compared SEDs of such candidates with YSO models to assess the likelihood of them being bona fide YSOs. Owing to brighter magnitude cuts to alleviate contamination from galaxies, their list of YSO candidates with high confidence recovers subsets of 9 and 4 in our lists of 26 primary YSOs and 83 fainter YSO candidates, respectively. Nonetheless, they identify three additional YSO candidates with colors bluer than our selection criteria: Y954, Y969, and Y970 (nomenclature from Sewiło et al. 2013). These three candidates are in a corner defined by $1.5<[4.5]-[8.0]$ $<2.0$ and $10.0<[8.0]<12.0$ : Y969 has an SED similar to our YSOs, whereas Y954 and Y970 have SEDs consistent with $\mathrm{B}[\mathrm{e}]$ supergiants but of fainter luminosities (Bonanos et al. 2010), requiring additional data to assess their nature as $\mathrm{Be}$ stars or YSOs. The much smaller number of YSO candidates with $[4.5]-[8.0]<2.0$ than YSO candidates with redder colors reported in the Sewiło et al. (2013) study lends credence that our criteria select the majority of YSOs in the Bridge.

\section{DETERMINING YSO PROPERTIES FROM MODEL FITS OF SEDs}

\subsection{Modeling the SEDs}

To infer the probable range of physical parameters for a YSO, we compare the observed SEDs with those from a model grid and select the best-fit models using $\chi^{2}$ minimization. We use the large grid from Robitaille et al. (2006) that includes 20,000 pre-calculated dust radiative transfer models, each containing a central star (a photospheric emitter of pre-main-sequence or main-sequence spectrum) surrounded by a flared circumstellar disk and a flattened rotating envelope with bipolar cavities. The best-fit models for observed SEDs is determined using the code from Robitaille et al. (2007) that requires fluxes and their errors of a YSO as the input. The fluxes of an SED are converted from magnitudes in Tables 1 and 2 . When calculating uncertainties associated with fluxes, we include errors from measurement (in Tables 1 and 2) and absolute flux calibration (i.e., $10 \%$ in each band from $B$ to $K_{s}, 5 \%$ in each band from 3.6 to $8.0 \mu \mathrm{m}, 10 \%$ in $24 \mu \mathrm{m}$, and $20 \%$ in $70 \mu \mathrm{m}$; Hambly et al. 2001b; Skrutskie et al. 2006; Kato et al. 2007; IRAC Data Handbook; MIPS Data Handbook) and estimate the total uncertainty of a flux using the quadratic sum of these two errors. The 26 YSOs in our sample have been analyzed with SED fitting. As the models are calculated for single YSOs, comparisons are plausible to the 17 YSOs that appear single or are clearly the dominant sources within the IRAC PSF. The remaining nine YSOs have multiple sources only resolved at some wavelengths. Although the total fitted luminosity of each group or multiple is robust, differences in color between the members may indicate different evolutionary stages; we discuss cases of such sources in Sections 5.2.1 and 5.2.2.

The results of SED fitting to the 26 YSOs are shown in Figure 6, with the best-fit and acceptable models overplotted on the SED of each YSO. The 26 YSOs are shown in the following order: first the 17 "single" YSOs arranged by order of increasing Types from our empirical classification and within each Type by order of increasing [8.0] magnitude, and then the 9 "multiple" YSOs arranged simply by order of increasing [8.0] magnitude. The best-fitting model for each source is determined by the minimum $\chi^{2}\left(\chi_{\min }^{2}\right)$, but there is typically a range of models 
which are nearly as consistent with the data, i.e., $\chi^{2}$ not significantly greater. We have used a cutoff of $\chi^{2}-\chi_{\min }^{2} \leqslant 3$ per datapoint for these acceptable models. The figure shows that uncertainty often results from parts of the SED at longer or shorter wavelengths than can be constrained by our data. Table 3 lists the results of model fits for the 26 YSOs which are tabulated in the same order as Figure 6. This table includes the source name, [8.0] magnitude, and type from our empirical classification, and weighted averages and standard deviations of selected physical parameters: central stellar mass $\left(M_{\star}\right)$, total luminosity $\left(L_{\text {tot }}\right)$, envelope accretion rate $\left(\dot{M}_{\text {env }}\right)$, disk mass $\left(M_{\text {disk }}\right)$, central stellar age $\left(\tau_{\star}\right)$, foreground extinction $\left(A_{V}\right)$, and inclination angle. The derived $\dot{M}_{\text {env }}$ and $M_{\text {disk }}$ have been scaled by a factor of 3.5 to account for the higher gas-to-dust ratio in clusters in the Bridge, which is likely to be representative for the YSO-forming regions, than the Galactic value used in YSO models (Gordon et al. 2009). These averages and standard deviations show a possible range of these physical parameters; they are calculated from best-fit and acceptable models using the inverse square of $\chi^{2}$ as the weight. For each accepted model, the evolutionary stage is determined using $\dot{M}_{\text {env }} / M_{\star}$ and $M_{\text {disk }} / M_{\star}$ ratios as defined in Robitaille et al. (2006), i.e., Stage I $-\dot{M}_{\text {env }} / M_{\star}>10^{-6} \mathrm{yr}^{-1}$; Stage II $-\dot{M}_{\text {env }} / M_{\star}<$ $10^{-6} \mathrm{yr}^{-1}$ and $M_{\text {disk }} / M_{\star}>10^{-6}$; and Stage III $-M_{\text {env }} / M_{\star}<$ $10^{-6} \mathrm{yr}^{-1}$ and $M_{\text {disk }} / M_{\star}<10^{-6}$. The range of the evolutionary stage, Stage Range, is the weighted standard deviation of the stages determined from each of the acceptable models for a YSO.

The model SEDs in general fit well with the observed SEDs of the 17 "single" YSOs (Figure 6), though discrepancies are seen in a number of the Type II YSOs. Several Type II YSOs have lower observed than modeled $4.5 \mu \mathrm{m}$ fluxes. This $4.5 \mu \mathrm{m}$ brightness dip is most likely a result of unaccounted contribution from PAH emission at 3.3, 6.2, 7.7, and $8.6 \mu \mathrm{m}$ in the other three IRAC bands. Since PAH emission features were not included in these pre-calculated models, in SED fits they are compensated by an increase in small grain continuum emission. However, our detailed analysis for YSOs in the LMC with and without correction of PAH emission demonstrates that this effect does not alter the derived physical parameters more than their quoted uncertainties (Chen et al. 2010). PAH emission has been detected in $87 \%$ of IRS spectra of 277 YSOs in the LMC and $80 \%$ of IRS spectra of 5 YSOs in the Bridge (Seale et al. 2009; $\mathrm{R}$. Indebetouw et al., in preparation). Bright PAH emission is likely also responsible for the YSOs with observed lower than modeled fluxes at both 4.5 and $24 \mu \mathrm{m}$ in SEDs.

Second, a few Type II YSOs have observed fluxes brighter than modeled fluxes at optical wavelengths. As massive YSOs evolve, compact $\mathrm{H}$ II regions typically form, but their small sizes $\sim 0.1-1 \mathrm{pc}$, or $\sim 0^{\prime} .4-4^{\prime \prime}$ in the Bridge, can only be resolved in high-resolution $\mathrm{H} \alpha$ images. Such cases have been demonstrated in massive YSOs in the LMC using Hubble Space Telescope or $4 \mathrm{~m}$ MOSAIC H $\alpha$ images (e.g., Chen et al. 2009, 2010; Vaidya et al. 2009). The optical BRI photometry were adopted from the SSS catalog based on broadband images taken with a CCD camera of a 0. ' $^{\prime} 67$ pixel $^{-1}$ scale and a resolution of $\sim 1$ 1'.5 (Hambly et al. 2001a). This would be inadequate to resolve central stars from surrounding compact $\mathrm{H}$ II regions of size $\lesssim 2^{\prime \prime}$. Bright nebular emission such as $\mathrm{H} \alpha$ would have raised the fluxes in $R$ and resulted in deviation from the dust radiative transfer models.

The most robust physical parameters derivable from model fitting are the total luminosity and circumstellar dust mass; the correspondingly derived stellar mass and evolutionary state are robust as well when single-YSO models are applicable. There is excellent agreement found between those stellar luminosities and masses inferred from SED fits of YSOs and from ionizing fluxes of corresponding ultra-compact $\mathrm{H}$ II regions in the LMC (Chen et al. 2009, 2010). For YSOs whose SEDs show discrepancies in the optical wavelengths, total luminosity can remain reliable for an SED relatively well reproduced in the MIR segment, as shown by YSOs 052207.3-675819.9 in N 44 and 054004.40-694437.6 in N 159 (Chen et al. 2009, 2010). In the models, circumstellar dust is distributed in a disk and a rotating flattened envelope. Although the relative distribution between those two components is difficult to constrain without (sub)millimeter photometry, the total mass of circumstellar dust is relatively robust. In cases where compact $\mathrm{H}$ II regions already formed around YSOs, the dust may be considered circumstellar in the SED fits rather than the more likely interstellar origin, and the inferred $\dot{M}_{\text {env }}$ and $M_{\text {disk }}$ may be overestimated. This is most likely in those sources with observed optical emission greater than the model, which as previously noted we believe to be likely optical nebular contamination.

\subsection{Evolutionary Stage of YSOs}

\subsubsection{Comparisons between Empirical "Type" and Model-derived "Stage" Classifications}

There is not yet a well-defined classification system for massive YSOs, since neither their formation mechanisms nor their circumstellar mass distribution is well-known. In the Robitaille et al. (2006) study, we proposed a "Stage" classification based on the physical quantities $M_{\text {env }}$ and $M_{\text {disk }}$, derived from the bestfitting models for a given source. Solar-mass YSO classifications have traditionally been based on the infrared spectral index, or infrared color excess. However, extension of the scheme to massive YSOs must be carefully parameterized, because a change in the central source's photospheric temperature and luminosity changes the emitted spectrum of heated circumstellar dust independently of the mass of circumstellar dust (Whitney et al. $2004 \mathrm{~b}$ ). Using the model-derived ratio of envelope to central source mass takes into account these effects in a physically self-consistent way.

At the distance of the Bridge, multiple sources or small clusters would be unresolved by Spitzer; thus, the circumstellar dust geometry may be complex and not correspond very well with these single-YSO models. As the physical conditions and structure of the surrounding ISM change as YSOs evolve, we proposed an empirical classification "Type" that uses both SEDs and immediate environmental morphology on sub-parsec scale to assess the evolutionary states of massive YSOs in the Magellanic system (Chen et al. 2009). There is also uncertainty in the "Type" classification as it assumes that the environmental morphology corresponds tightly to the evolutionary state of a single or dominant source. Comparisons between the two schemes can help to better understand the amount and distribution of dust around massive YSOs in the Bridge and to obtain a comprehensive picture of their evolution.

The analysis of 17 "single" YSOs is used to compare the two classifications Type and Stage. As listed in Table 3, types and stages are not overwhelmingly correlated. Upon close examination, among the seven Types II or II/III YSOs (relatively evolved) that have inferred Stage $\sim 1.0-1.2$ (relatively unevolved), five show observed optical fluxes higher than model SEDs, implying presence of nebular emission and hence $\mathrm{H}$ II regions likely to have formed. The MIR dust emission from 
Table 3

Inferred Physical Parameters from SED Fits to YSOs

\begin{tabular}{|c|c|c|c|c|c|c|c|c|c|c|}
\hline Source Name & $\begin{array}{l}{[8.0]} \\
(\mathrm{mag})\end{array}$ & Type & $\begin{array}{l}\text { Stage } \\
\text { Range }\end{array}$ & $\begin{array}{c}\bar{M}_{*} \\
\left(M_{\odot}\right) \\
\end{array}$ & $\begin{array}{c}\bar{L}_{\text {tot }} \\
\left(L_{\odot}\right) \\
\end{array}$ & $\begin{array}{c}\dot{\bar{M}}_{\mathrm{env}} \\
\left(M_{\odot} \mathrm{yr}^{-1}\right) \\
\end{array}$ & $\begin{array}{l}\bar{M}_{\text {disk }} \\
\left(R_{\odot}\right) \\
\end{array}$ & $\begin{array}{c}\overline{\tau_{*}} \\
(\mathrm{yr})\end{array}$ & $\begin{array}{c}\overline{A_{V}} \\
(\mathrm{mag})\end{array}$ & $\begin{array}{c}\text { incl. } \\
\left({ }^{\circ}\right)\end{array}$ \\
\hline J014914.81-733944.20 & 10.14 & I & $1.5 \pm 0.5$ & $6.6 \pm 1.2$ & $1.3 \mathrm{E}+03 \pm 9.7 \mathrm{E}+02$ & $3.2 \mathrm{E}-04 \pm 3.1 \mathrm{E}-04$ & $4.5 \mathrm{E}-01 \pm 3.5 \mathrm{E}-01$ & $4.3 \mathrm{E}+05 \pm 4.3 \mathrm{E}+05$ & $13.1 \pm 9.3$ & $38.8 \pm 21.8$ \\
\hline J014536.69-741258.78 & 9.58 & $\mathrm{I} / \mathrm{II}$ & $1.6 \pm 0.5$ & $9.6 \pm 2.6$ & $6.4 \mathrm{E}+03 \pm 5.6 \mathrm{E}+03$ & $1.7 \mathrm{E}-04 \pm 2.2 \mathrm{E}-04$ & $3.3 \mathrm{E}-01 \pm 2.7 \mathrm{E}-01$ & $9.6 \mathrm{E}+05 \pm 8.0 \mathrm{E}+05$ & $17.2 \pm 12.8$ & $45.2 \pm 19.8$ \\
\hline J015045.05-742337.17 & 11.21 & $\mathrm{I} / \mathrm{II}$ & $1.1 \pm 0.3$ & $6.2 \pm 1.0$ & $5.1 \mathrm{E}+02 \pm 2.8 \mathrm{E}+02$ & $2.2 \mathrm{E}-03 \pm 1.6 \mathrm{E}-03$ & $4.5 \mathrm{E}-01 \pm 3.7 \mathrm{E}-01$ & $1.2 \mathrm{E}+05 \pm 2.0 \mathrm{E}+05$ & $0.9 \pm 1.0$ & $23.4 \pm 15.1$ \\
\hline J020214.18-741210.61 & 11.71 & $\mathrm{I} / \mathrm{II}$ & $1.6 \pm 0.5$ & $5.1 \pm 1.0$ & $4.7 \mathrm{E}+02 \pm 2.0 \mathrm{E}+02$ & $6.8 \mathrm{E}-04 \pm 6.6 \mathrm{E}-04$ & $1.4 \mathrm{E}-01 \pm 1.1 \mathrm{E}-01$ & $1.6 \mathrm{E}+06 \pm 2.0 \mathrm{E}+06$ & $1.3 \pm 1.8$ & $40.9 \pm 21.7$ \\
\hline J021526.72-740432.77 & 9.05 & II & $1.0 \pm 0.0$ & $9.1 \pm 0.8$ & $2.0 \mathrm{E}+03 \pm 7.3 \mathrm{E}+02$ & $5.2 \mathrm{E}-04 \pm 3.9 \mathrm{E}-04$ & $2.1 \mathrm{E}-01 \pm 1.6 \mathrm{E}-01$ & $4.0 \mathrm{E}+04 \pm 2.9 \mathrm{E}+04$ & $1.5 \pm 1.6$ & $29.6 \pm 16.6$ \\
\hline J020503.24-735303.19 & 9.64 & II & $1.0 \pm 0.0$ & $7.9 \pm 0.8$ & $1.5 \mathrm{E}+03 \pm 5.6 \mathrm{E}+02$ & $9.5 \mathrm{E}-04 \pm 7.4 \mathrm{E}-04$ & $2.3 \mathrm{E}-01 \pm 1.8 \mathrm{E}-01$ & $9.6 \mathrm{E}+04 \pm 4.0 \mathrm{E}+04$ & $1.1 \pm 0.8$ & $27.4 \pm 11.5$ \\
\hline J021505.12-743954.86 & 9.78 & II & $1.0 \pm 0.0$ & $7.5 \pm 0.4$ & $1.3 \mathrm{E}+03 \pm 2.5 \mathrm{E}+02$ & $5.9 \mathrm{E}-04 \pm 4.9 \mathrm{E}-04$ & $2.1 \mathrm{E}-01 \pm 1.7 \mathrm{E}-01$ & $1.4 \mathrm{E}+05 \pm 5.4 \mathrm{E}+04$ & $0.6 \pm 0.6$ & $37.1 \pm 16.6$ \\
\hline J015435.23-742646.24 & 10.88 & II & $1.7 \pm 0.5$ & $6.2 \pm 1.0$ & $1.1 \mathrm{E}+03 \pm 8.4 \mathrm{E}+02$ & $2.4 \mathrm{E}-05 \pm 2.2 \mathrm{E}-05$ & $9.8 \mathrm{E}-02 \pm 1.1 \mathrm{E}-01$ & $9.7 \mathrm{E}+05 \pm 9.8 \mathrm{E}+05$ & $0.6 \pm 0.5$ & $48.7 \pm 19.1$ \\
\hline J015315.03-744510.22 & 10.88 & II & $1.0 \pm 0.0$ & $6.2 \pm 0.9$ & $5.1 \mathrm{E}+02 \pm 2.0 \mathrm{E}+02$ & $1.4 \mathrm{E}-04 \pm 1.2 \mathrm{E}-04$ & $5.9 \mathrm{E}-01 \pm 4.5 \mathrm{E}-01$ & $1.8 \mathrm{E}+05 \pm 1.0 \mathrm{E}+05$ & $0.8 \pm 0.9$ & $40.2 \pm 17.8$ \\
\hline J021948.20-743517.55 & 11.21 & II & $1.1 \pm 0.3$ & $6.0 \pm 0.8$ & $5.0 \mathrm{E}+02 \pm 1.2 \mathrm{E}+02$ & $8.6 \mathrm{E}-05 \pm 6.2 \mathrm{E}-05$ & $7.1 \mathrm{E}-01 \pm 5.2 \mathrm{E}-01$ & $3.4 \mathrm{E}+05 \pm 2.2 \mathrm{E}+05$ & $1.7 \pm 1.4$ & $48.0 \pm 15.0$ \\
\hline J014705.47-742601.50 & 11.40 & II & $2.2 \pm 1.0$ & $8.0 \pm 2.2$ & $3.5 \mathrm{E}+03 \pm 3.0 \mathrm{E}+03$ & $2.0 \mathrm{E}-04 \pm 2.9 \mathrm{E}-04$ & $1.7 \mathrm{E}-01 \pm 1.5 \mathrm{E}-01$ & $1.8 \mathrm{E}+06 \pm 1.4 \mathrm{E}+06$ & $1.6 \pm 1.0$ & $53.4 \pm 22.4$ \\
\hline J015857.18-740954.93 & 11.67 & II & $2.0 \pm 0.1$ & $8.2 \pm 1.8$ & $3.8 \mathrm{E}+03 \pm 3.1 \mathrm{E}+03$ & $8.0 \mathrm{E}-05 \pm 1.9 \mathrm{E}-04$ & $3.9 \mathrm{E}-02 \pm 4.3 \mathrm{E}-02$ & $2.3 \mathrm{E}+06 \pm 1.0 \mathrm{E}+06$ & $36.5 \pm 12.0$ & $56.6 \pm 20.7$ \\
\hline J015518.06-743529.29 & 11.78 & II & $1.9 \pm 0.3$ & $5.0 \pm 0.7$ & $4.7 \mathrm{E}+02 \pm 1.6 \mathrm{E}+02$ & $4.7 \mathrm{E}-05 \pm 1.3 \mathrm{E}-04$ & $1.1 \mathrm{E}-01 \pm 8.9 \mathrm{E}-02$ & $2.4 \mathrm{E}+06 \pm 2.0 \mathrm{E}+06$ & $1.6 \pm 0.5$ & $48.9 \pm 18.9$ \\
\hline J014838.65-744441.71 & 11.02 & II/III & $1.0 \pm 0.0$ & $6.2 \pm 0.5$ & $5.6 \mathrm{E}+02 \pm 1.5 \mathrm{E}+02$ & $9.9 \mathrm{E}-05 \pm 7.2 \mathrm{E}-05$ & $6.6 \mathrm{E}-01 \pm 4.9 \mathrm{E}-01$ & $2.7 \mathrm{E}+05 \pm 6.4 \mathrm{E}+04$ & $0.6 \pm 0.5$ & $41.9 \pm 11.7$ \\
\hline J020552.13-740445.44 & 11.75 & II/III & $1.9 \pm 0.3$ & $4.4 \pm 0.5$ & $3.0 \mathrm{E}+02 \pm 6.4 \mathrm{E}+01$ & $1.1 \mathrm{E}-05 \pm 1.2 \mathrm{E}-05$ & $1.5 \mathrm{E}-01 \pm 1.2 \mathrm{E}-01$ & $9.2 \mathrm{E}+05 \pm 2.1 \mathrm{E}+05$ & $1.2 \pm 0.5$ & $46.8 \pm 17.4$ \\
\hline J021915.53-745300.02 & 11.83 & II/III & $1.9 \pm 0.3$ & $4.4 \pm 0.5$ & $3.0 \mathrm{E}+02 \pm 6.8 \mathrm{E}+01$ & $1.3 \mathrm{E}-05 \pm 1.3 \mathrm{E}-05$ & $1.5 \mathrm{E}-01 \pm 1.2 \mathrm{E}-01$ & $9.1 \mathrm{E}+05 \pm 2.3 \mathrm{E}+05$ & $0.9 \pm 0.4$ & $43.6 \pm 16.9$ \\
\hline J020744.59-745302.97 & 11.90 & II/III & $1.2 \pm 0.4$ & $5.2 \pm 0.6$ & $4.4 \mathrm{E}+02 \pm 1.1 \mathrm{E}+02$ & $5.1 \mathrm{E}-05 \pm 3.8 \mathrm{E}-05$ & $2.0 \mathrm{E}-01 \pm 1.6 \mathrm{E}-01$ & $5.6 \mathrm{E}+05 \pm 2.6 \mathrm{E}+05$ & $1.2 \pm 0.7$ & $43.6 \pm 18.3$ \\
\hline J021246.35-744040.26 & 8.97 & mul & $1.0 \pm 0.0$ & $8.4 \pm 0.9$ & $1.8 \mathrm{E}+03 \pm 5.8 \mathrm{E}+02$ & $1.2 \mathrm{E}-03 \pm 1.0 \mathrm{E}-03$ & $3.8 \mathrm{E}-01 \pm 2.9 \mathrm{E}-01$ & $8.2 \mathrm{E}+04 \pm 4.0 \mathrm{E}+04$ & $0.3 \pm 0.3$ & $32.0 \pm 13.0$ \\
\hline J022152.32-744537.83 & 9.12 & mul & $1.0 \pm 0.0$ & $9.5 \pm 1.0$ & $2.3 \mathrm{E}+03 \pm 7.2 \mathrm{E}+02$ & $5.5 \mathrm{E}-03 \pm 4.1 \mathrm{E}-03$ & $4.3 \mathrm{E}-01 \pm 3.4 \mathrm{E}-01$ & $4.4 \mathrm{E}+04 \pm 4.4 \mathrm{E}+04$ & $1.1 \pm 1.0$ & $19.5 \pm 4.0$ \\
\hline J021936.75-741929.38 & 9.59 & mul & $1.0 \pm 0.0$ & $7.5 \pm 0.4$ & $1.4 \mathrm{E}+03 \pm 2.4 \mathrm{E}+02$ & $5.5 \mathrm{E}-04 \pm 4.6 \mathrm{E}-04$ & $1.0 \mathrm{E}-01 \pm 8.7 \mathrm{E}-02$ & $1.4 \mathrm{E}+05 \pm 5.4 \mathrm{E}+04$ & $0.1 \pm 0.2$ & $29.2 \pm 9.9$ \\
\hline J014353.94-743224.71 & 9.71 & mul & $1.0 \pm 0.0$ & $8.1 \pm 1.0$ & $1.7 \mathrm{E}+03 \pm 5.4 \mathrm{E}+02$ & $2.6 \mathrm{E}-03 \pm 2.1 \mathrm{E}-03$ & $6.3 \mathrm{E}-01 \pm 5.2 \mathrm{E}-01$ & $1.1 \mathrm{E}+05 \pm 5.8 \mathrm{E}+04$ & $0.0 \pm 0.0$ & $18.2 \pm 0.0$ \\
\hline J014942.43-743704.76 & 10.21 & mul & $1.0 \pm 0.1$ & $8.2 \pm 1.7$ & $1.5 \mathrm{E}+03 \pm 8.5 \mathrm{E}+02$ & $5.7 \mathrm{E}-03 \pm 4.3 \mathrm{E}-03$ & $3.7 \mathrm{E}-01 \pm 3.1 \mathrm{E}-01$ & $8.2 \mathrm{E}+04 \pm 1.8 \mathrm{E}+05$ & $7.9 \pm 8.4$ & $22.3 \pm 11.1$ \\
\hline J022152.99-744734.94 & 10.23 & mul & $2.3 \pm 0.8$ & $10.0 \pm 0.9$ & $6.5 \mathrm{E}+03 \pm 1.9 \mathrm{E}+03$ & $2.0 \mathrm{E}-05 \pm 1.9 \mathrm{E}-05$ & $1.0 \mathrm{E}-01 \pm 9.6 \mathrm{E}-02$ & $1.4 \mathrm{E}+06 \pm 8.8 \mathrm{E}+05$ & $0.1 \pm 0.1$ & $69.5 \pm 22.1$ \\
\hline J014929.21-743916.48 & 10.73 & mul & $1.2 \pm 0.4$ & $7.8 \pm 1.1$ & $1.3 \mathrm{E}+03 \pm 4.6 \mathrm{E}+02$ & $4.3 \mathrm{E}-03 \pm 3.2 \mathrm{E}-03$ & $3.0 \mathrm{E}-01 \pm 2.5 \mathrm{E}-01$ & $1.4 \mathrm{E}+05 \pm 2.9 \mathrm{E}+05$ & $10.9 \pm 8.9$ & $26.3 \pm 16.7$ \\
\hline J021440.18-742127.03 & 10.79 & mul & $1.8 \pm 0.4$ & $10.4 \pm 1.1$ & $6.4 \mathrm{E}+03 \pm 2.3 \mathrm{E}+03$ & $1.5 \mathrm{E}-03 \pm 1.6 \mathrm{E}-03$ & $8.3 \mathrm{E}-02 \pm 1.0 \mathrm{E}-01$ & $6.9 \mathrm{E}+05 \pm 3.2 \mathrm{E}+05$ & $35.6 \pm 9.5$ & $50.2 \pm 23.1$ \\
\hline J020649.68-744359.95 & 10.97 & $\mathrm{mul}$ & $1.7 \pm 0.4$ & $6.1 \pm 0.6$ & $1.1 \mathrm{E}+03 \pm 3.8 \mathrm{E}+02$ & $1.8 \mathrm{E}-05 \pm 1.6 \mathrm{E}-05$ & $6.2 \mathrm{E}-02 \pm 7.3 \mathrm{E}-02$ & $1.8 \mathrm{E}+06 \pm 1.7 \mathrm{E}+06$ & $1.0 \pm 0.6$ & $48.2 \pm 19.9$ \\
\hline
\end{tabular}


Table 4

Inferred Physical Parameters from SED Fits to Fainter YSO Candidates

\begin{tabular}{|c|c|c|c|c|c|c|c|c|}
\hline ource Name & $\begin{array}{c}\bar{M}_{*} \\
\left(M_{\odot}\right)\end{array}$ & $\begin{array}{c}\bar{L}_{\mathrm{tot}} \\
\left(L_{\odot}\right)\end{array}$ & $\begin{array}{c}\dot{M}_{\mathrm{env}} \\
\left(M_{\odot} / \mathrm{yr}\right)\end{array}$ & $\begin{array}{l}\bar{M}_{\text {disk }} \\
\left(R_{\odot}\right)\end{array}$ & $\begin{array}{l}\text { Stage } \\
\text { Range }\end{array}$ & $\begin{array}{c}\overline{\tau_{*}} \\
(\mathrm{yr})\end{array}$ & $\begin{array}{c}\overline{A_{V}} \\
(\mathrm{mag})\end{array}$ & $\begin{array}{c}i \bar{c} \overline{c l} . \\
\left({ }^{\circ}\right)\end{array}$ \\
\hline & $5.6 \pm 1.5$ & $.0 \mathrm{E}+03 \pm 1.0 \mathrm{E}+03$ & $5.5 \mathrm{E}-05 \pm 1.1 \mathrm{E}-04$ & $6 \mathrm{~F}-02+50 \mathrm{~F}-02$ & & $2.8 \mathrm{E}+06 \pm 1.7 \mathrm{E}+06$ & $34.7 \pm 13.8$ & $49.2 \pm 22.3$ \\
\hline J014003.4 & $5.9 \pm 1.6$ & $1.2 \mathrm{E}+03 \pm 1.7 \mathrm{E}+03$ & $3 E-05 \pm 1.5 E-05$ & $\mathrm{E}-02 \pm 5.9$ & $1.7 \pm$ & $7 \mathrm{E}+05 \pm 6.9$ & $1.0=$ & $42.9 \pm 19.2$ \\
\hline J014011.01-740303.42 & $5.1 \pm 1.7$ & $5.2 \mathrm{E}+02 \pm 5.0 \mathrm{E}+02$ & $3.8 \mathrm{E}-04 \pm 4.3 \mathrm{E}-04$ & $1.9 \mathrm{E}-01 \pm 1.5 \mathrm{E}-01$ & $1.4 \pm 0.5$ & $9.8 \mathrm{E}+05 \pm 1.4 \mathrm{E}+06$ & $1.9 \pm 2.4$ & $40.7 \pm 21.0$ \\
\hline J014109.05-743704.11 & $3.5 \pm 1.7$ & $1.8 \mathrm{E}+02 \pm 4.0 \mathrm{E}+02$ & $1.9 \mathrm{E}-04 \pm 2.0 \mathrm{E}-04$ & $1.1 \mathrm{E}-01 \pm 9.1 \mathrm{E}-02$ & $1.2 \pm 0.4$ & $5.0 \mathrm{E}+05 \pm 1.1 \mathrm{E}+06$ & $20.0 \pm 14.9$ & $42.4 \pm 19.2$ \\
\hline J014110.65-743748.07 & $5.1 \pm 1.2$ & $5.9 \mathrm{E}+02 \pm 9.8 \mathrm{E}+02$ & $5.9 \mathrm{E}-05 \pm 1.1 \mathrm{E}-04$ & $5.9 \mathrm{E}-02 \pm 5.4 \mathrm{E}-02$ & $1.7 \pm 0.5$ & $2.0 \mathrm{E}+06 \pm 2.0 \mathrm{E}+06$ & $18.8 \pm 10.6$ & $50.0 \pm 20.9$ \\
\hline
\end{tabular}

(This table is available in its entirety in a machine-readable form in the online journal. A portion is shown here for guidance regarding its form and content.)

unresolved H II regions included in the YSO SED is interpreted by the models as circumstellar disks or envelopes even though it is unlikely to accrete onto the sources (however see, e.g., Keto 2007, for a discussion of accretion of ionized gas in massive protostars). When excluding these five Types II or II/III YSOs, there is a rough trend between types and stages as Types I and I/II YSOs have Stages $\leqslant 1.6$ and Types II and II/III have Stages $\sim 2.0$.

Neither Type III nor Stage III YSOs are found in the Bridge, unlike in LMC H II complexes (Chen et al. 2009, 2010). This may be partly due to the lack of high-resolution $\mathrm{H} \alpha$ images that are used in the Type scheme to discriminate Type III from Type II. Our exclusion of unresolved, extended sources with double-peaked galaxy-like SEDs (Section 3.1.1) also may exclude some Type III YSOs, but this effect should not be different between the LMC and Bridge. On the other hand, it is possible that the Type/Stage III phase is shorter or exhibiting different observational properties compared to those in the LMC. Stage III corresponds to transitional and debris disks, and a faster dust disk dissipation time at lower metallicity (harder stellar field, lower interstellar dust-to-gas ratio) is not implausible. Further comparisons between YSOs in the Bridge and the LMC are discussed in Section 4.4.

\subsubsection{Evolutionary Stages of Faint YSO Candidates}

The fainter YSO candidates were selected from the lower part of the [8.0] versus ([4.5]-[8.0]) CMD (Figure 3) that is populated by YSOs that are more evolved or have lower masses than those in the upper part of the CMD. To infer the probable ranges of physical parameters of these fainter YSO candidates, we have compared their SEDs to YSO models. The results of model fits of the 83 fainter candidates are given in Table 4 , including the source name, weighted average and standard deviation of selected physical parameters: $M_{\star}, L_{\mathrm{tot}}$, $\dot{M}_{\text {env }}, M_{\text {disk }}$, Stage, $\tau_{\star}, A_{V}$, and inclination angle. The weighted average and standard deviation are calculated using the same procedure described in Section 4.1. As shown in Figure 8, fainter YSO candidates have more advanced evolutionary states with $77 \%$ being Stage II (weighted average stage $=1.5-2.5$ ) and $3 \%$ being Stage III (weighted average stage $\geqslant 2.5$ ), more evolved than our primary YSO sample with $46 \%$ being Stage II and zero Stage III. These results are consistent with what is expected for populations in these two parts of the CMD.

\subsubsection{Evolutionary Stages of HAeBe Candidates}

As YSOs have evolved from the embedded phases and revealed their stellar photospheres, their circumstellar envelopes might have mostly dissipated and only remnant disks remain. Thus the NIR excess is likely more prominent than MIR excess. HAeBe are young stars of intermediate masses that are frequently identified by their NIR excess (e.g., Hillenbrand et al. 1992). Since their mass range overlaps with our YSO sample, we examine whether some of them might be previously identified as $\mathrm{HAeBe}$ candidates and, if so, how their evolutionary stages are compared to our YSOs. Using the IRSF $J H K_{s}$ catalog, Nishiyama et al. (2007) selected 200 HAeBe candidates in the Bridge based on their NIR excess in $J H K_{s}$ CMDs. Such candidates are of modest reliability, as NIR excess can originate from not only circumstellar dust around young stars, but also interstellar dust near main-sequence and giant stars, or background galaxies (e.g., Nakajima et al. 2005). In this list of HAeBe candidates in the Bridge, Nishiyama et al. (2007) estimated $\sim 60 \%$ contaminants.

With our multi-wavelength SEDs and images, we have demonstrated that background galaxies can be identified, as can main-sequence or giant stars obscured by cool $(\sim 30 \mathrm{~K})$ interstellar dust, opposed to YSOs or evolved stars with warm $(\geqslant 100 \mathrm{~K})$ circumstellar dust. To assess the nature of the HAeBe candidates in the Bridge, we constructed their multi-wavelength SEDs by matching them with our photometric catalog. When matching sources, we allow a $1^{\prime \prime}$ error margin. Among the 203 HAeBe candidates in the Nishiyama et al. (2007) list, 199 are in our working field, and 191 of them have matches in our catalog. We compare SEDs of these 191 sources to YSO models as well as stellar models from Brott \& Hauschildt (2005) for stellar temperature $T_{\star}<10,000 \mathrm{~K}$ and from Castelli \& Kurucz (2004) for $T_{\star} \geqslant 10,000 \mathrm{~K}$; these stellar models are included in the SED fitting code (Robitaille et al. 2007). SEDs of main-sequence or giant stars should be well reproduced by stellar models reddened by interstellar dust without obvious MIR excess in the IRAC or MIPS bands, for example, HAeBe candidate J014226.34-741432.13 shown in Figure 7. SEDs of stars with (warm) circumstellar dust would show IRAC or MIPS fluxes higher than stellar models reddened by interstellar dust and would be relatively well reproduced by YSO models, such as HAeBe candidate J014744.02-742551.95 in Figure 7. Based on these comparisons, we have identified 96 HAeBe candidates in the Nishiyama et al. (2007) list that show IRAC flux excess. These sources are most likely HAeBe stars, though a fraction of them could be more evolved, such as Be stars that also have warm circumstellar dust.

The results of model fits of the $96 \mathrm{HAeBe}$ candidates are given in Table 5, including the source name, weighted average and standard deviation of selected physical parameters: $M_{\star}$, $L_{\text {tot }}, \dot{M}_{\text {env }}, M_{\text {disk }}$, Stage, $\tau_{\star}, A_{V}$, and inclination angle. The weighted average and standard deviation are calculated using the same procedure described in Section 4.1. 84\% of these HAeBe candidates have more advanced evolutionary states (Figure 8), with $55 \%$ being Stage III and $29 \%$ being Stage II, more evolved than the Stages I and II (with the maximum weighted average Stage $=2.3$ ) inferred for most of the YSOs selected using 

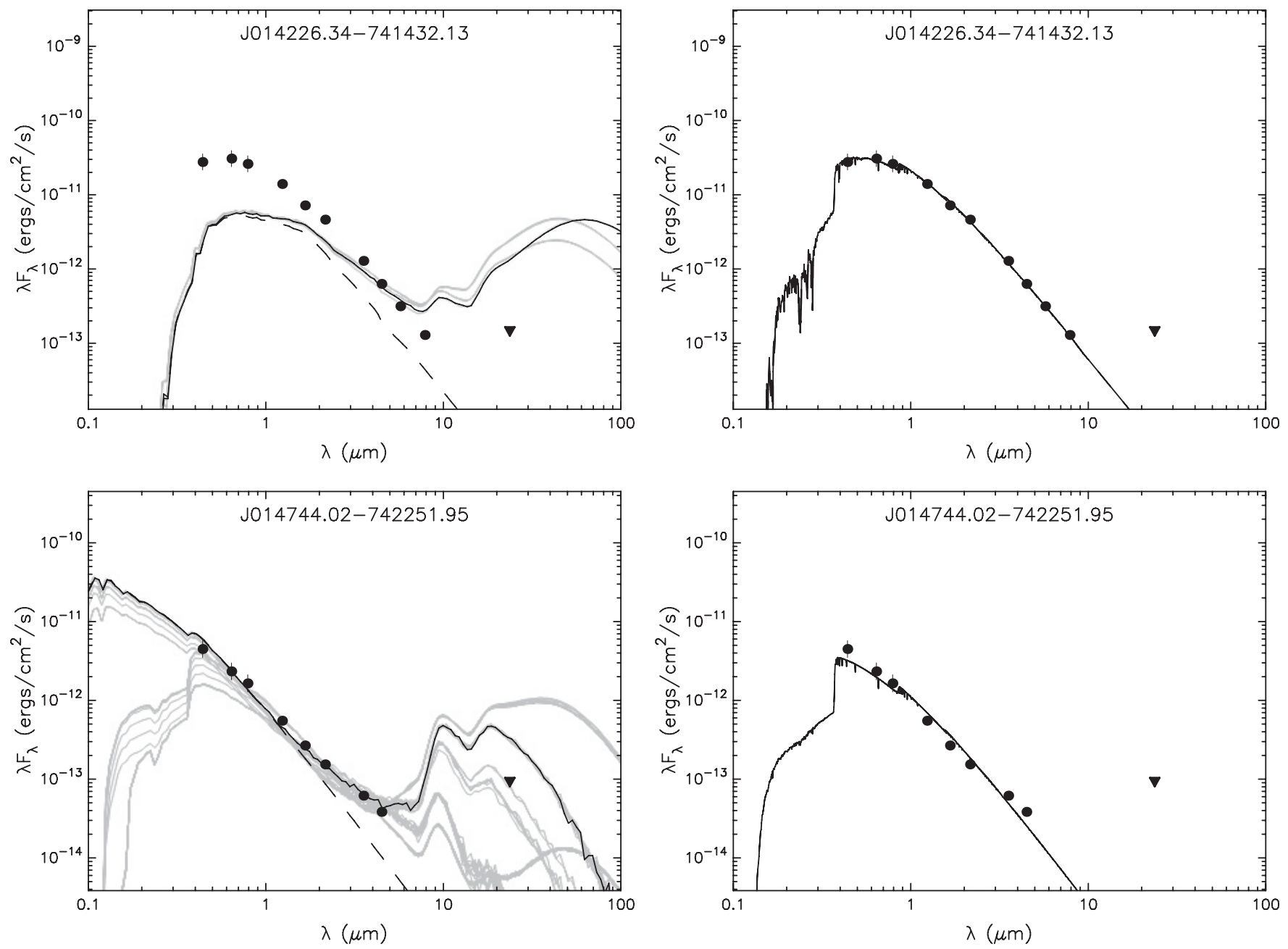

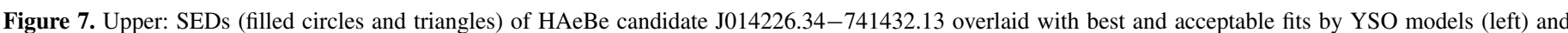
single best fit by stellar models (right). Symbols are the same as Figure 6. Lower: the same setup of HAeBe candidate J014744.02-742551.95.

Table 5

Inferred Physical Parameters from SED Fits to HAeBe Candidates

\begin{tabular}{|c|c|c|c|c|c|c|c|c|}
\hline Source Name & $\begin{array}{c}\bar{M}_{*} \\
\left(M_{\odot}\right)\end{array}$ & $\begin{array}{c}\bar{L}_{\text {tot }} \\
\left(L_{\odot}\right)\end{array}$ & $\begin{array}{c}\overline{\dot{M}}_{\text {env }} \\
\left(M_{\odot} / \mathrm{yr}\right)\end{array}$ & $\begin{array}{l}\bar{M}_{\text {disk }} \\
\left(R_{\odot}\right)\end{array}$ & $\begin{array}{l}\text { Stage } \\
\text { Range }\end{array}$ & $\begin{array}{c}\overline{\bar{\tau}_{*}} \\
(\mathrm{yr})\end{array}$ & $\begin{array}{c}\overline{A_{V}} \\
(\mathrm{mag})\end{array}$ & $\begin{array}{c}i n \bar{c} l . \\
\left({ }^{\circ}\right)\end{array}$ \\
\hline & $5.5 \pm 0.4$ & $26 \mathrm{~F}+02+63 \mathrm{~F}+01$ & $9 \mathrm{E}-05 \pm 1.4 \mathrm{E}-05$ & $1.7 \mathrm{E}-03 \pm 1.5 \mathrm{E}-03$ & & $3.5 \mathrm{E}+05 \pm 1.2 \mathrm{E}+05$ & $.0 \pm 0.0$ & \\
\hline J014039.65-743243.22 & & & $9.4 \mathrm{E}-06 \pm 1.3 \mathrm{E}-0$ & & & & & $49.9 \pm 19.7$ \\
\hline $014050.82-741030.86$ & $14.6 \pm 0.0$ & $1.9 \mathrm{E}+04 \pm 0.0 \mathrm{E}+00$ & $0.0 \mathrm{E}-00 \pm 0.0 \mathrm{E}-00$ & $3.2 \mathrm{E}-08 \pm 2.3 \mathrm{E}-08$ & $3.0 \pm 0.0$ & $1.3 \mathrm{E}+06 \pm 4.7 \mathrm{E}+00$ & $0.0 \pm 0.0$ & $57.6 \pm 21.0$ \\
\hline J014142.53-735528.92 & $14.6 \pm 0.0$ & $1.9 \mathrm{E}+04 \pm 0.0 \mathrm{E}+00$ & $0.0 \mathrm{E}-00 \pm 0.0 \mathrm{E}-00$ & $3.2 \mathrm{E}-08 \pm 2.3 \mathrm{E}-08$ & $3.0 \pm 0.0$ & $1.3 \mathrm{E}+06 \pm 0.0 \mathrm{E}+00$ & $0.0 \pm 0.0$ & $57.8 \pm 21.0$ \\
\hline J014221.85-743013.85 & $5.0 \pm 0.2$ & $3.0 \mathrm{E}+02 \pm 6.8 \mathrm{E}+01$ & $8.0 \mathrm{E}-06 \pm 6.1 \mathrm{E}-06$ & $6.6 \mathrm{E}-02 \pm 5.3 \mathrm{E}-02$ & $1.3 \pm 0.5$ & $5.4 \mathrm{E}+05 \pm 1.1 \mathrm{E}+05$ & $0.0 \pm 0.0$ & $40.6 \pm 16.3$ \\
\hline
\end{tabular}

(This table is available in its entirety in a machine-readable form in the online journal. A portion is shown here for guidance regarding its form and content.)

MIR CMDs. Note that the list of HAeBe candidates from Nishiyama et al. (2007) does not overlap with our YSO list, contrasted with LMC H II complex N 159 where lists of HAeBe candidates and Spitzer-selected YSOs do overlap (Chen et al. 2010). This disjointedness of the two samples in the Bridge is mostly attributed to bluer NIR color cuts Nishiyama et al. (2007) used to select HAeBe candidates than were used in the LMC. These bluer color cuts were based on the argument in the de Wit et al. (2003) study that the amount of circumstellar dust around HAeBe stars at low metallicity would be much less and hence results in smaller NIR color excess and bluer colors, though they also found that all their bluer candidates have ambiguous natures as their light curves and spectral features are also consistent with post main-sequence Be stars. If the NIR color cuts that had been used in N 159 (Nakajima et al. 2005) were applied to select HAeBe candidates in the Bridge, three candidates indeed would overlap with our YSOs and have an evolutionary state of Stage II. The reliability of a list of NIRselected HAeBe candidates can be greatly improved using multiwavelength SEDs, but the definitive identification of these more evolved YSOs and understanding the evolution of circumstellar disks requires spectroscopic and/or variability studies.

\subsection{Masses of YSOs, Fainter YSO Candidates, and $\mathrm{HAeBe}$ Candidates}

The mass estimates, i.e., the $\chi^{2}$-weighted average mass $\bar{M}_{*}$ from the best and acceptable fits, of 26 YSOs in the Bridge are 

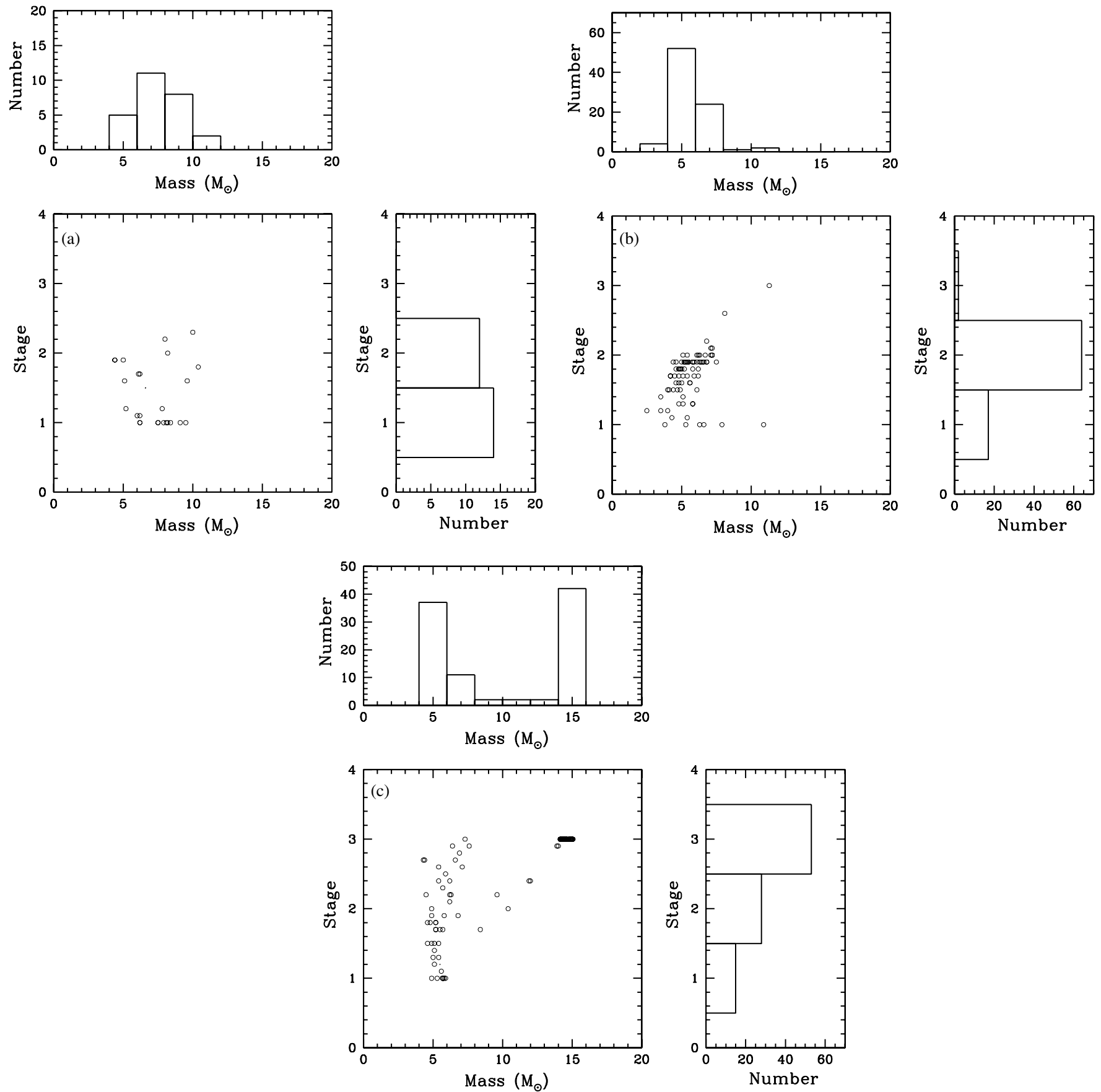

Figure 8. Stage vs. mass inferred from SED fits for (a) 26 YSOs, (b) 83 fainter YSO candidates, and (c) 96 HAeBe candidates in the Bridge. Histograms of mass and Stage are plotted on the sides. These three types of sources show different dominant populations of Stages: YSOs, fainter YSO candidates, and HAeBe candidates have their dominant populations in Stages I, II, and III, respectively, consistent with expectations for these sources. YSOs and faint YSO candidates have masses ranging 4-11 $M_{\odot}$. Between the two YSO samples, fainter YSO candidates have masses mostly in the lower end of the range, though the two most massive ones have masses $\sim 11 M_{\odot}$, comparable to those in our primary YSO sample. HAeBe candidates show a bimodal distribution of masses, with the high-mass $\left(\sim 15 M_{\odot}\right)$ population all having more evolved evolutionary stage (Stage III).

given in Table 3. The mass estimates would be reliable for the 17 "single" YSOs as their SEDs can be properly approximated by single-YSO models. By contrast, the other nine have SEDs from multiple YSOs that might not be well reproduced by singleYSO models; their mass estimates have larger uncertainties. The results of the SED fits, as illustrated in Figure 8(a), show that ten YSOs in the Bridge have $\bar{M}_{*} \geqslant 8 M_{\odot}$; these are most likely bona fide massive YSOs. The remaining 16 YSOs have $\bar{M}_{*}<8 M_{\odot}$; these are likely intermediate-mass YSOs. The Bridge does not contain embedded YSOs as massive as active regions in the
LMC, which contain O-type embedded YSOs with masses 17-45 $M_{\odot}$ (e.g., Chen et al. 2009, 2010). The most massive embedded YSOs in the Bridge are $\sim 10 M_{\odot}$, corresponding to $\sim$ B2 stars.

The mass estimates from the best and acceptable fits of 83 fainter YSO candidates in the Bridge are listed Table 4. Their masses are mostly in the lower end of the mass range (Figure 8(b)): $80(=96 \%)$ of the fainter YSO candidates have masses $<8 M_{\odot}$ with the peak of the distribution falling in the 4-6 $M_{\odot}$ mass bin. That most sources are in this low-mass bin is 

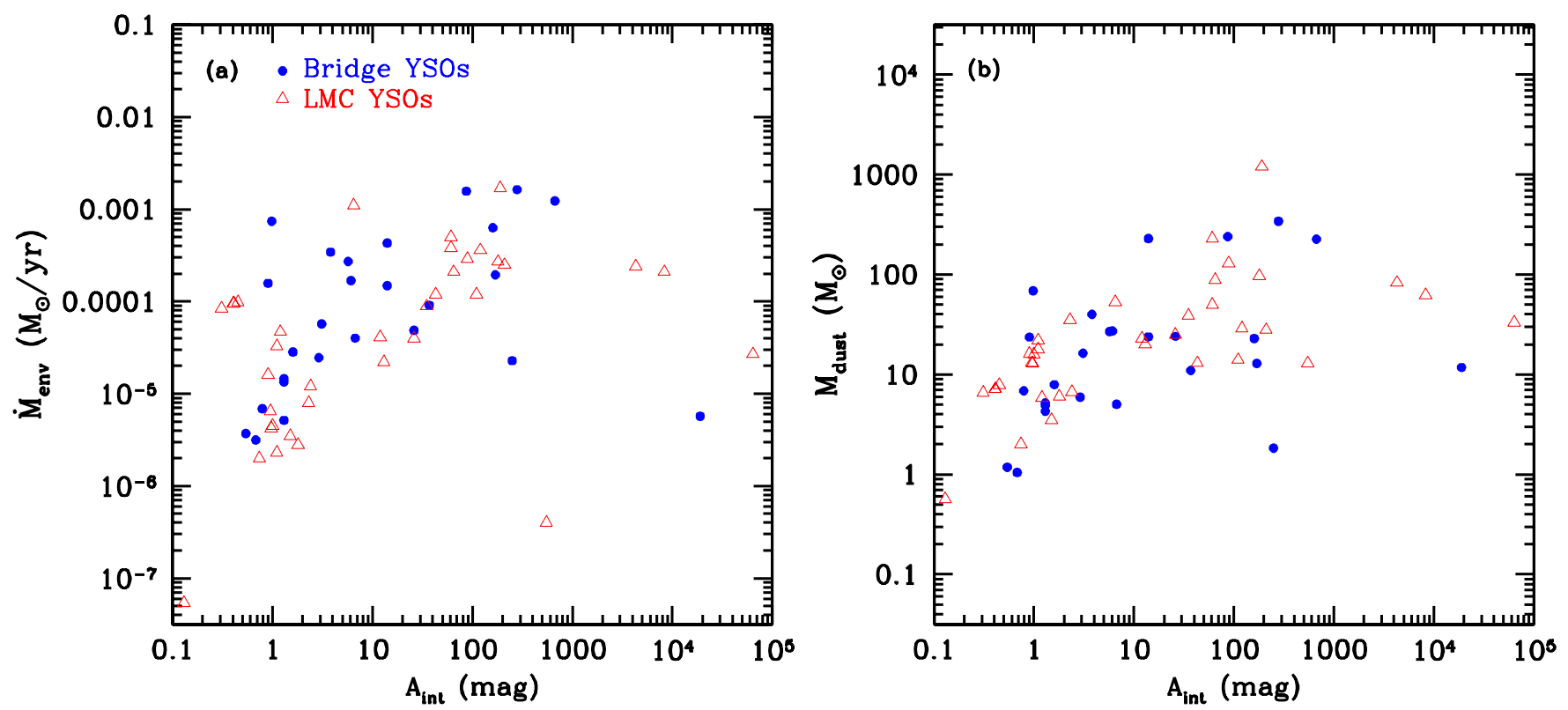

Figure 9. (a) $A_{\text {int }}$ vs. $\dot{M}_{\text {env }}$ and (b) $A_{\text {int }}$ vs. $M_{\text {dust }}$ of YSOs in the Bridge (filled circles). For comparison, YSOs in two LMC H II complexes (open triangles; Chen et al. $2009,2010)$ are also plotted. $M_{\text {env }}$ and $M_{\text {dust }}$ in this figure are not scaled with respective gas-to-dust ratios for the LMC and Bridge so that the comparisons among parameters are all based on dust masses. YSOs in the Bridge show a similar distribution in the inferred amount of circumstellar dust compared to in the LMC, even though the optical counterparts are more frequently detected in the former than the latter.

(A color version of this figure is available in the online journal.)

expected for sources in the fainter part of the CMD. However, three of these fainter sources have masses $\geqslant 8 M_{\odot}$, with two of them up to $\sim 11 M_{\odot}$, comparable to the most massive in our primary sample. The fainter [8.0] magnitudes (compared to our primary YSO sample) of these three massive YSOs are likely attributed to a more edge-on viewing angle, $57^{\circ}-86^{\circ}$ as inferred from the SED fitting (Table 4). Among these three fainter YSO candidates with masses $\geqslant 8 M_{\odot}$, the most massive two are found in Cloud $\mathrm{C}$ and the third one in Cloud A.

The mass estimates from the best and acceptable fits of 96 $\mathrm{HAeBe}$ candidates in the Bridge are listed Table 5. The masses of the HAeBe candidates show a bimodal distribution, with 50 candidates in the mass range $4-10 M_{\odot}$ and 46 in $10-16 M_{\odot}$ (Figure $8(\mathrm{c})$ ). Candidates in the lower-mass peak have a mass range comparable to the embedded YSOs, implying that the most massive stars formed in the Bridge in the last several Myr are $\sim 10 M_{\odot}$. Candidates in the higher-mass end (14-16 $M_{\odot}$, corresponding to B1V) are all Stage III sources with ages $\gtrsim 3 \mathrm{Myr}$, though a fraction of them are likely much older Be stars, of spectral type B1 and often found in clusters with ages ranging from $\gtrsim 3$ to $30 \mathrm{Myr}$ (Grebel 1997). Comparisons between these mass ranges of YSOs and HAeBe candidates show that the Bridge has not been actively producing O-type stars in the last several Myr and that the intensity of massive star formation appears to have decreased with time, as the most massive stars formed $\gtrsim 3 \mathrm{Myr}$ ago are $\sim 15 M_{\odot}$, while those formed currently are only $\sim 10 M_{\odot}$.

\subsection{Comparisons of YSO Properties at Different Metallicity}

It has been suggested that a lower dust abundance and greater permittivity to ultraviolet radiation of the ISM is expected to affect pre-formation gas dynamics, as well as cooling and feedback from massive YSOs (Poglitsch et al. 1995). The Bridge and the LMC have different metallicities, $1 / 5-1 / 8$ and $1 / 3 Z_{\odot}$, respectively, and thus provide an excellent opportunity to examine the metallicity effect on massive star formation. We compare the properties of YSOs in the Bridge to those from our studies of two LMC H II complexes, N 44 and N 159 (Chen et al. 2009, 2010). These two complexes have 41 YSOs in the mass range overlapping with YSOs in the Bridge, i.e., 4-11 $M_{\odot}$. Comparisons among them show that YSOs in the Bridge appear less embedded, as $81 \%(=21 / 26)$ of them show optical counterparts, while only $56 \%(=23 / 41)$ in these two LMC complexes. The difference is even larger when taking into account that SSS BRI images of the Bridge are much shallower than our 4 m MOSAIC deep UBVI images of LMC complexes, allowing fewer optical counterparts to be detected. The higher frequency of optical counterparts implies a smaller extinction in YSOs in the Bridge, likely due to its lower dust content in either the molecular clouds or circumstellar envelopes, or both.

We investigate several possible causes of the higher frequency of optical counterparts for YSOs in the Bridge. First, we examine if the extinction difference is mostly from molecular clouds by comparing foreground extinction $A_{V}$ (fitted in our SED modeling) in these YSOs. We find similar values in the Bridge and the LMC complexes, i.e., $A_{V}=3.4 \pm 8.9$ and $3.5 \pm 7.0$, respectively, suggesting that molecular clouds are not the main cause in the extinction difference. To search for differences in circumstellar dust, we compare the central source mass $M_{\star}$, line-of-sight extinction due to dust in the circumstellar envelope $A_{\text {int }}$, the envelope accretion rate $\dot{M}_{\text {env }}$, and total amount of circumstellar mass $M_{\text {dust }} . A_{\text {int }}$ is determined from the entire SED, while $\dot{M}_{\text {env }}$ and $M_{\text {dust }}$ mostly depend on MIR emission. As shown in Figure 9, there is no obvious difference in these parameters between YSOs in the Bridge and those in LMC H II complexes. Note that since $A_{\text {int }}$ depends on dust mass, not dust + gas mass, $\dot{M}_{\text {env }}$ and $M_{\text {dust }}$ in this figure are thus not scaled with respective gas-to-dust ratios for the LMC and Bridge so that comparisons among these parameters can be all on dust masses. If circumstellar material had a smooth spherical distribution, then the circumstellar line-of-sight extinction $A_{\text {int }}$ would be 


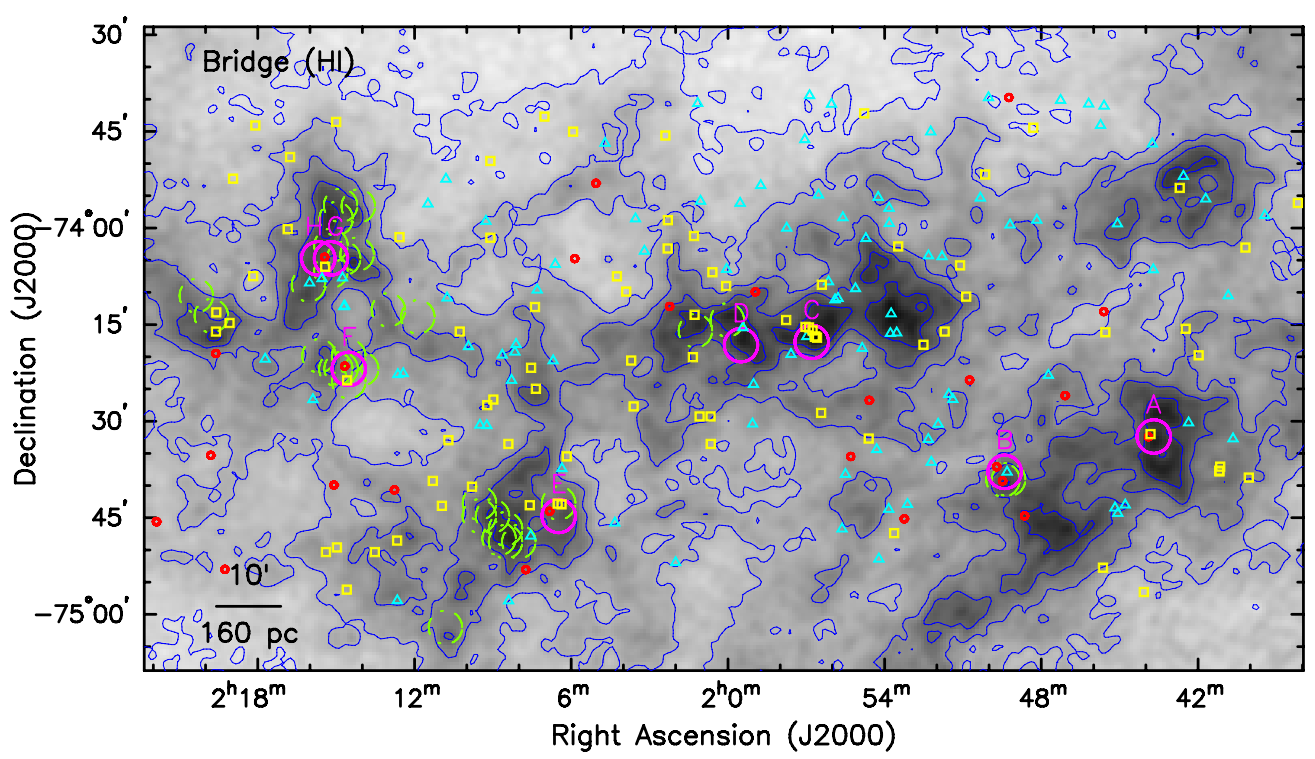

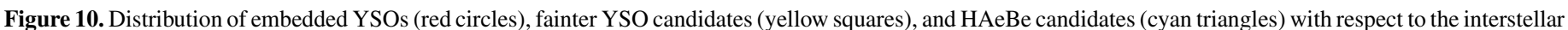

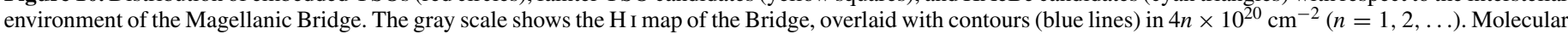

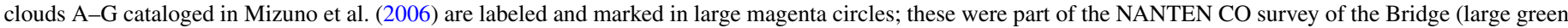
circles; Y. Fukui et al., in preparation).

(A color version of this figure is available in the online journal.)

directly related to the circumstellar dust mass $M_{\text {dust }}$ and derived envelope accretion rate $\dot{M}_{\text {env }}$. However, for a nonspherical (disklike or clumpy/irregular) circumstellar dust distribution, the line-of-sight extinction is primarily related to the viewing angle (inclination angle for a disk-like geometry, presence or absence of a dense clump on the line of sight for a clumpy geometry, see e.g., Indebetouw et al. 2006). The derived dust mass, however, is primarily related to the total MIR to FIR emission, and less sensitive to the geometry. Finally, the presence of a bright optical counterpart corresponds to how clumpy the distribution is overall (or for a disk, the size of the bipolar openings) - a more porous distribution of the same amount of dust will allow more short-wavelength light to scatter out and be observed. What we see in this comparison is that Bridge and LMC massive YSOs have similar circumstellar dust masses, that the presence or absence of a line-of-sight clump is not particularly different, but that overall the envelopes of Bridge YSOs are likely more porous or clumpy than in LMC YSOs. This may also imply that these YSOs have a shorter timescale for dispersal of circumstellar material and a more rapid YSO evolution.

\section{MASSIVE STAR FORMATION IN THE MAGELLANIC BRIDGE}

A causal relationship between the physical conditions of interstellar environment and formation of massive stars is not easy to establish since once massive stars are formed, their strong UV radiation and fast stellar winds ionize and disperse the natal clouds and subsequently alter the physical conditions of the ambient ISM. Embedded massive YSOs, on the other hand, have not had time to significantly affect their surrounding medium beyond parsec scales, providing an excellent chance to probe issues on massive star formation. We use the massive YSOs found in the Bridge to investigate issues such as the relationship between star formation properties and interstellar conditions and the progression of star formation in the tidal environment.

\subsection{Interstellar Environments and Star Formation Properties}

How massive stars are formed in a region and whether there is a dependence on environmental factors such as metallicity and tidal interaction are crucial to the understanding of star formation across the near and far universe. To study these questions, one of the most direct ways is to examine the relationship between massive YSOs and their natal environment, particularly the molecular environment as the dense clumps are where stars are formed. We thus examine the properties of massive YSOs in the Bridge and their relation to molecular clouds. Eight molecular clouds have been detected toward the Bridge from Mopra, SEST, and NANTEN CO $J=1-0$ surveys (Muller et al. 2003b; Mizuno et al. 2006). This census of molecular clouds is not complete; these low-metallicity clouds require brightness temperature sensitivities of $\lesssim 10 \mathrm{mK}$ to detect, so the search was limited to regions selected primarily based on bright IRAS $100 \mu \mathrm{m}$ emission (regions observed are marked in Figure 10, and the search criteria described in detail in Mizuno et al. 2006 and Y. Fukui et al., in preparation). These IRAS sources have a significant fraction of contaminants such as background galaxies (recall Section 3.1.1 on culling out such contaminants from the list of YSO candidates), or diffuse dust regions unresolved with IRAS $2^{\prime}$-resolution $(\sim 30 \mathrm{pc}$ in the Bridge). Furthermore, as the majority of the YSOs in the Bridge are intermediate masses and thus too faint for IRAS surveys, it is not surprising that we find numerous embedded YSOs not covered by previous $\mathrm{CO}$ surveys and hence not associated with detected CO.

Despite the small size (eight) and incompleteness of the existing CO cloud sample, all but one have associated embedded YSOs; the only one without YSOs, Cloud D, has the faintest $\mathrm{CO}$ emission with a marginal $2 \sigma$ detection (Mizuno et al. 2006). This tight spatial correlation between the molecular clouds and YSOs implies that star formation happens quickly once these clouds are formed. By contrast, star formation does not appear to happen swiftly in the large group (36) of massive clouds in the molecular ridge of the LMC as very few of them show massive 

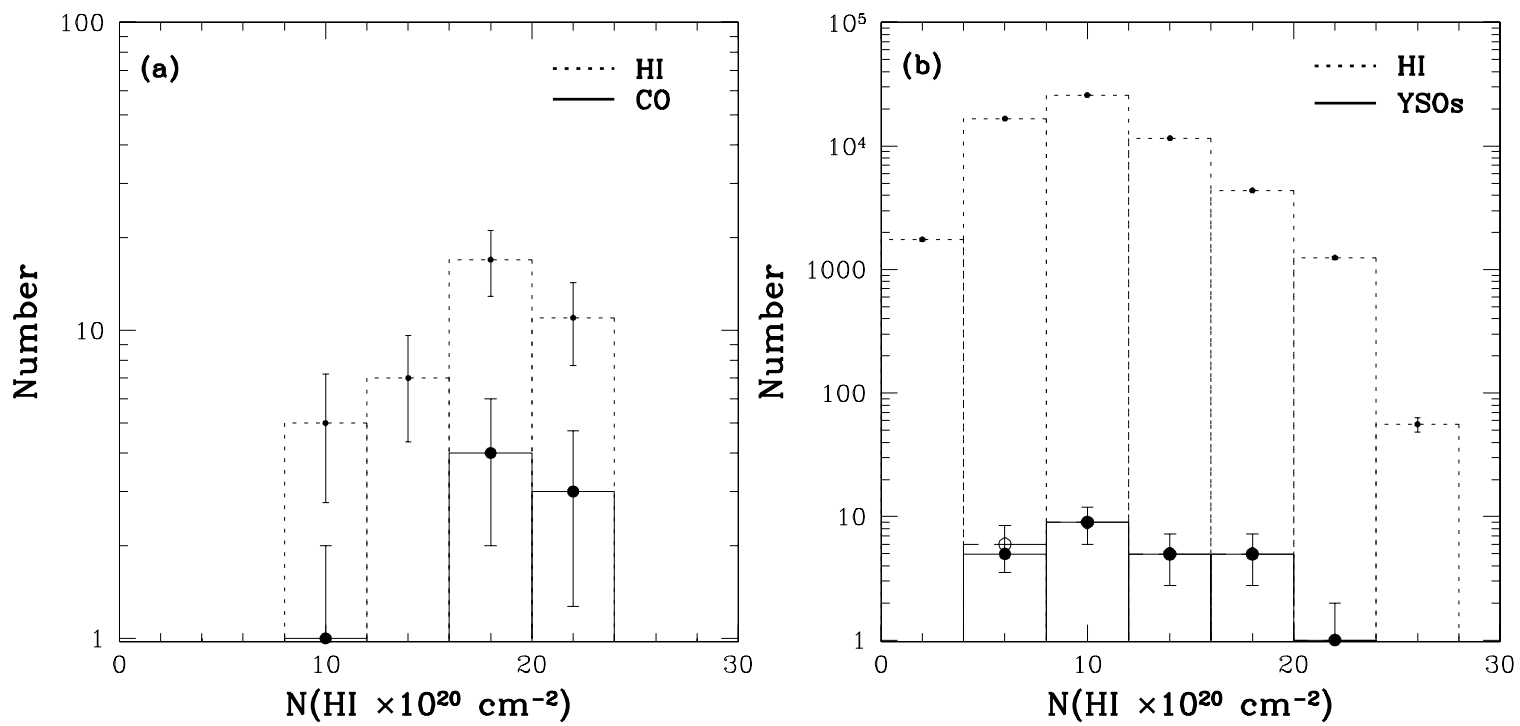

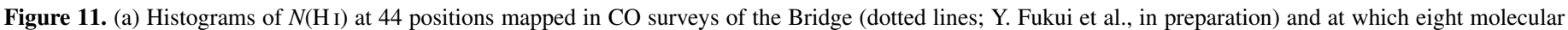

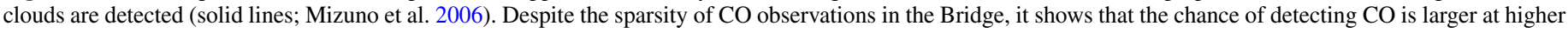
$N(\mathrm{HI})$. (b) Histograms of all H I resolution elements (dotted lines) and those containing YSOs (solid lines) in the Bridge.

star formation activity in the past $\gtrsim 10$ Myr or host massive YSOs (Indebetouw et al. 2008). The eight Bridge clouds exhibit an extensive range of massive stars formed in the past $\gtrsim 10 \mathrm{Myr}$ and at present; we discuss the propagation of star formation in these clouds individually in Section 5.2.

To better understand the formation mechanisms of the entire YSO sample in the Bridge, we compare the distribution of YSOs to $\mathrm{H}$ I emission, as $N(\mathrm{HI})$ indicates where the bulk of atomic gas is, and the ATCA H I survey has mapped the entire Bridge we studied (Muller et al. 2003a). As shown in Figure 10, the $N(\mathrm{HI})$ in the Bridge ranges up to $2.7 \times 10^{21} \mathrm{~cm}^{-2}$; compared to the LMC and SMC that have maximum $N(\mathrm{HI})=8.8$ and $14.3 \times$ $10^{21} \mathrm{~cm}^{-2}$, respectively (Kim et al. 2003; Stanimirovic et al. $1999)$, the $N(\mathrm{HI})$ distribution of the Bridge overlaps with the lower end of the LMC and SMC. Molecular clouds (and stars forming from them) tend to be found in regions with higher $N(\mathrm{HI}$ ), as shown in the LMC and SMC (Wong et al. 2009; Leroy et al. 2007; Muller et al. 2010), and also in the small sample of molecular clouds in the Bridge (Figure 11). Thus, it is not unexpected that the majority, $>70 \%$, of the YSOs in the Bridge are found in $N(\mathrm{HI}) \geqslant 8 \times 10^{20} \mathrm{~cm}^{-2}$ (Figure 10). We estimate the mass of molecular material that may be associated with such a column of $\mathrm{HI}$ : at first glance, this $N(\mathrm{HI})$ appears to correspond to a very low $\mathrm{A}_{V}<0.1$ for a gas-to-dustratio $N(\mathrm{HI}) / \mathrm{A}_{V} \sim 10-32 \times 10^{21} \mathrm{~cm}^{-2} \mathrm{mag}^{-1}$ found in the nearby SMC H II region N 83 (Leroy et al. 2009) or the $30 \times$ $10^{21} \mathrm{~cm}^{-2} \mathrm{mag}^{-1}$ measured in the Bridge (Gordon et al. 2009). This is much lower than $\mathrm{A}_{V} \sim 0.5-1$ needed for the surface $\mathrm{H}_{2}$ formation rate to effectively balance ultraviolet photodestruction (Hartmann et al. 2001). However, considering the Hi map's 98" -resolution ( $\sim 26 \mathrm{pc}$ in the Bridge) and $\mathrm{Hr}: \mathrm{H}_{2}=4: 1$ (Dame 1993), $N(\mathrm{H} \mathrm{I}) \sim 8 \times 10^{20} \mathrm{~cm}^{-2}$ could hide a $5 \mathrm{pc}$ sized molecular cloud with gas surface density $\sim 6.7 \times 10^{21} \mathrm{~cm}^{-2}$, corresponding to $\mathrm{A}_{V} \sim 0.2-0.7$ for the aforementioned gas-to-dust-ratio. Such a molecular cloud would have a mass $\sim 1300 M_{\odot}$.

To quantify the SFE in the Bridge, we examine $\epsilon$, the fraction of $\mathrm{H}$ I resolution elements containing YSOs as a function of $N(\mathrm{HI})$. This is equivalent to the probability of finding YSOs at a given $N(\mathrm{HI})$. Figure 11 shows histograms of the Bridge's H I resolution elements, i.e., $30^{\prime \prime}$ pixel $^{-1}$, and YSOs, as well as $\epsilon$ as a function of $N(\mathrm{HI})$. To examine clustering of YSOs, an additional histogram of YSOs is made to count those in the same H I pixel as one YSO. Figure 11 (open compared to filled circles) shows that the clustering is minimal since only one $\mathrm{H}$ I pixel in the Bridge contains two YSOs. $\epsilon$ of the Bridge appears to be higher at higher $N(\mathrm{HI})$, though there is no obvious linear correlation. To assess if star and cloud formation is affected by metallicity or galactic environment, we further compare $\epsilon$ between the Bridge and LMC. Histograms of the LMC's H I resolution elements in the same pixel scale of $30^{\prime \prime}$ and YSOs (Kim et al. 2003; Gruendl $\&$ Chu 2009) are shown in Figure 12, along with $\epsilon$ as a function of $N(\mathrm{HI})$. As in the Bridge, only a small fraction, $\lesssim 10 \%$, of H I pixels in the LMC contain multiple YSOs, so clustering on scales $<30^{\prime \prime}$ does not affect the result there either. Comparisons of $\epsilon$ show that the Bridge is $\lesssim 1 / 3$ the LMC in their overlapping bins of $N(\mathrm{HI})=12-24 \times 10^{20} \mathrm{~cm}^{-2}$. However, at lower $N(\mathrm{HI})$, the Bridge shows a flatter slope so that its $\epsilon$ is even up to $\sim 2$ times the LMC. To investigate if this flatter slope is a result of local variations within the LMC, we have also estimated $\epsilon$ for seven regions across the LMC with the same angular size as the Bridge, and 1-1.5 times the Bridge's average $N(\mathrm{HI})$. We found that all these regions may have $\epsilon$ higher or lower than the average $\epsilon$ of the entire LMC, but their slopes are always rising. The Bridge's flatter slope does indeed appear unique.

We examine the YSO properties in the LMC and the Bridge to investigate the possible causes of different $\epsilon$ in these two systems. The YSOs in the Bridge have inferred masses of 4-10 $M_{\odot}$, not as massive as those up to $45 M_{\odot}$ found in the LMC (e.g., Chen et al. 2009, 2010); as will be discussed in Section 5.3, the dearth of YSOs $\geqslant 10 M_{\odot}$ in the Bridge cannot be explained by stochastic effects. Furthermore, Bridge YSOs are sparsely distributed (Figure 10), in sharp contrast to LMC YSOs that are usually in groups and clusters (e.g., Chen et al. 2009, 2010; Gruendl \& Chu 2009). The lack of massive clusters in the Bridge is consistent with the simulations of Krumholz et al. (2009), in which cluster-forming molecular clouds are scarce at that density and metallicity. On the other hand, the reverse trend appears to suggest a different dominant mechanism of star formation at the lower $N(\mathrm{HI})$ regime. The sparse distribution of YSOs and low masses of molecular clouds, 

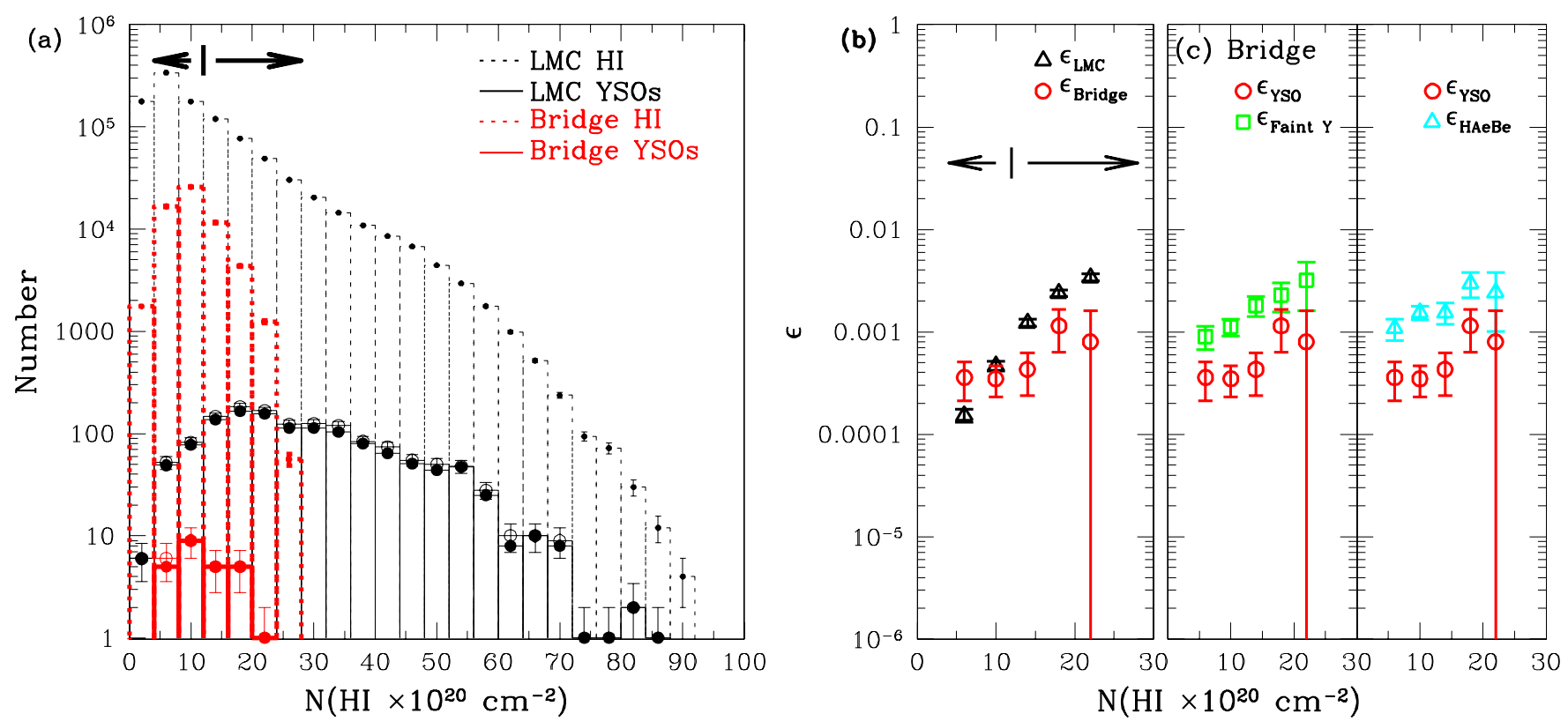

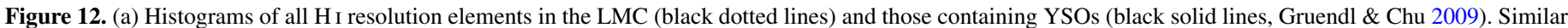

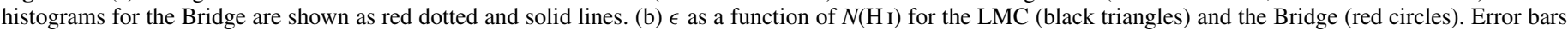

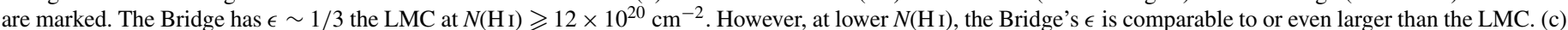

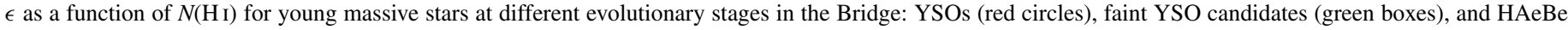
candidates (cyan triangles). The two older populations show a mild decrease with decreasing N(H I) and no flatter slopes in low N(H I) as seen in the YSOs.

(A color version of this figure is available in the online journal.)

$10^{3}-10^{4} M_{\odot}$, in the Bridge (Mizuno et al. 2006), as well as the rapid star formation implied by the tight spatial correlation between molecular clouds and YSOs, are consistent with star formation through colliding flows (Heitsch et al. 2006). In the Heitsch et al. (2006) simulations, Taurus-type molecular clouds (masses $\sim 1.5 \times 10^{4} M_{\odot}$, Pineda et al. 2010) are formed in colliding $\mathrm{H}$ I flows and followed by nearly instantaneous star formation. The comparisons in $\epsilon$ and star formation properties between the LMC and the Bridge indicate that at the higher $N(\mathrm{H}$ I) regime, the lower metallicity of the Bridge is likely responsible for lower efficiencies of star and molecular cloud formation. At the lower $N(\mathrm{HI})$ regime, the Bridge's dynamic tidal environment may enhance distributed star formation.

Finally, we have further examined if the flatter slope seen in $\epsilon$ calculated using the Bridge YSOs also appears in fainter YSO candidates or HAeBe candidates. Figure 12(c) illustrates $\epsilon$ determined for all three kinds of young massive stars in the Bridge. Unlike YSOs showing a flatter slope in $\epsilon$ toward low $N(\mathrm{HI})$, fainter YSO candidates and HAeBe candidates exhibit a monotonically mild decrease. This mild decrease might be attributed to quick dissipation of some clouds so that objects once formed in high $N(\mathrm{H}$ I $)$ now have lower $N(\mathrm{H}$ I $)$ surroundings. In this case, the dissipation timescale has to be shorter than the age of fainter YSO candidates and HAeBe candidates, i.e., a few Myr (Tables 4 and 5). We note that such a short dissipation timescale, if proven, is consistent with expectations from star formation through colliding flows.

\subsection{Star Formation in Individual Molecular Clouds}

To determine the mode of massive star formation, examine its progress in space and time and to assess if some might be triggered in the Bridge, we compare the underlying distributions of massive stars and YSOs in all eight molecular clouds detected from previous CO surveys (Figure 10: Muller et al. 2003b;
Mizuno et al. 2006). Each cloud is discussed individually in the following subsections, except that Cloud $\mathrm{H}$ is part of Cloud $\mathrm{G}$ and hence is included in the discussion of Cloud G. As previously mentioned in Section 5.1 that the majority of YSOs in the Bridge were not covered in previous CO surveys; we have carried out a new CO survey of all YSOs and will discuss the results in a forthcoming paper.

\subsubsection{Molecular Cloud A}

Star formation has been occurring around Molecular Cloud $\mathrm{A}$, as it is located within a large stellar association BS191 and is associated with two compact stellar clusters BS192 and BS193 (nomenclature from Bica \& Schmitt 1995). The evidence of massive stars formed in the last $10 \mathrm{Myr}$ is revealed by the presence of two faint, circular H II regions, each with size $\sim 100^{\prime \prime}$ or $\sim 27 \mathrm{pc}$ shown in the $\mathrm{H} \alpha$ image in Figure 13(a). It has been suggested that the $\mathrm{H}$ II regions are unlikely ionized by the aforementioned association and clusters based on spatial separation (Muller \& Parker 2007). Indeed, far- and nearultraviolet (FUV and NUV) images of the Bridge from the GALEX All-Sky Survey reveal three bright blue stars near the H II region centers (Figure 13(b)). These three stars are most likely the ionizing stars as they are the brightest and also the bluest within the H II regions. They have FUV $=14.37$, 14.39, and 15.28, and (FUV - NUV) $=-0.28,-0.17$, and -0.28 , respectively (GALEX Data Release 6); these magnitudes and colors are consistent with one to two B2V stars or up to one B1V star at $55 \mathrm{kpc}$, the distance of the Bridge, with little extinction, i.e., FUV = 15.0, 14.2, to 13.9, respectively, and the same $(\mathrm{FUV}-\mathrm{NUV})=-0.30$. Thus, these $\mathrm{H}$ iI regions appear to be ionized by single or double B2 or B1 stars, each with stellar mass $\sim 10$, up to $13 M_{\odot}$.

The current massive star formation in Molecular Cloud A is revealed by our Spitzer observations. Two YSOs are identified, an embedded YSO J014353.94-743224.71 and a fainter YSO 

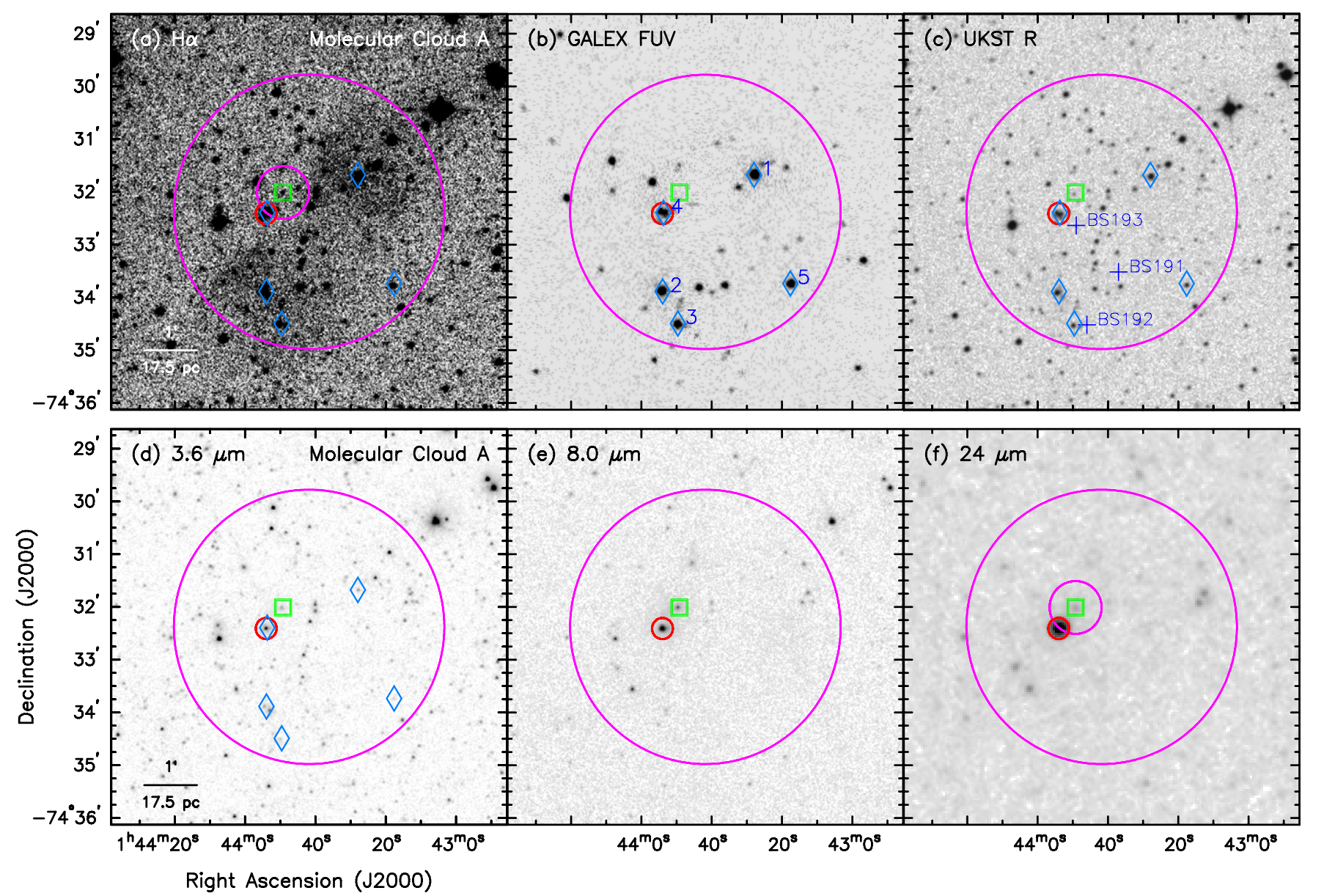

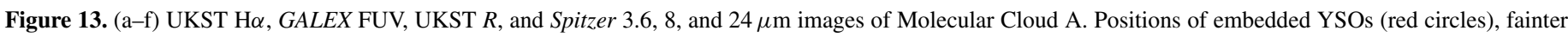

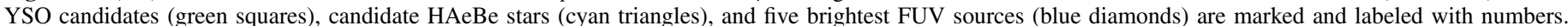

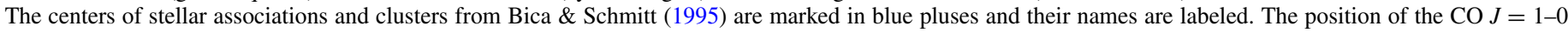

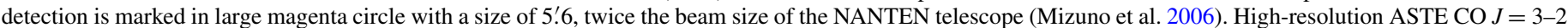

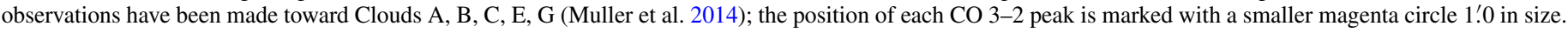
(A color version of this figure is available in the online journal.)

candidate J014349.20-743200.63 (Figure 13). Both YSOs have mass estimates $\sim 8 M_{\odot}$ (Tables 3 and 4 ) but show different stellar environments. The embedded YSO is in a compact multiple system containing massive stars of similar masses at different apparent evolutionary stages. As shown in Figures 3 (Section 3.1.2) and 13, the YSO, dominating the NIR and MIR light, is young with an estimated age of $0.11 \pm 0.06 \mathrm{Myr}$ (Table 4), while the FUV bright star, dominating the UV and blue light, has $\mathrm{FUV}=15.75$ and $(\mathrm{FUV}-\mathrm{NUV})=-0.11$ that are consistent with those of a B2-3V star (mass $\sim 8-10 M_{\odot}$ ), and hence has at least reached the zero-age main-sequence (ZAMS) and is older than a few Myr (Beech \& Mitalas 1994). In contrast, the fainter YSO in the cloud appears to have only one massive source. The fainter YSO has an age estimate of $2.6 \pm 1.2 \mathrm{Myr}$, much more evolved than the embedded YSO but still young enough to possess circumstellar dust and hence less likely reaching the ZAMS. This fainter YSO is at the $\mathrm{CO}$ peak of Cloud A revealed by $\sim 20^{\prime \prime}$-resolution ASTE CO observations (Muller et al. 2014).

Our examination of massive star and YSO populations in Molecular Cloud A shows that a handful of early B (B1-3) stars in small groups formed some 5-10 Myr ago. Current (most recent few Myr) star formation might have been triggered by the expansion of HiI regions as the YSOs are found near the edge of the Hil regions. Furthermore, the most massive star formed in the current generation is $\sim 8 M_{\odot}$, less massive than $\lesssim 13 M_{\odot}$ in the previous generation; this is consistent with the trend observed in Galactic and LMC H II regions that the being-triggered second generation is less massive than the first (Pomarès et al. 2009; Fleener et al. 2010; Chen et al. 2010). If the current star formation is indeed triggered and given that Cloud A's low mass of $1 \times 10^{3} M_{\odot}$ (Mizuno et al. 2006) is similar to the Taurus Cloud forming mostly low-mass stars, the star formation mode in Cloud A most likely will remain as distributed, small groups of B stars rather than a large OB association.

\subsubsection{Molecular Cloud B}

Star formation has been occurring in Molecular Cloud B as it contains a stellar association BS200 and a compact cluster WG3, and both are associated with nebular emission (Bica \& Schmitt 1995). The $\mathrm{H} \alpha$ image of Cloud B shows an arc filament overlapping with WG3 and a compact bright $\mathrm{H}$ II region spatially coincident with BS200 (Figure 14(a)). It has been suggested that the ionizing stars of these $\mathrm{H}$ II regions are likely from these stellar association and cluster (Muller \& Parker 2007), and the GALEX UV data reveal their nature and location more precisely (Figure 14(b)). The brightest star is at the center of the compact bright $\mathrm{H}$ II region coincident with $\mathrm{BS} 200$; it has $\mathrm{FUV}=13.71$ and $(\mathrm{FUV}-\mathrm{NUV})=-0.10$, consistent with a B1V star at $55 \mathrm{kpc}$. The second and third brightest stars are in the interior 

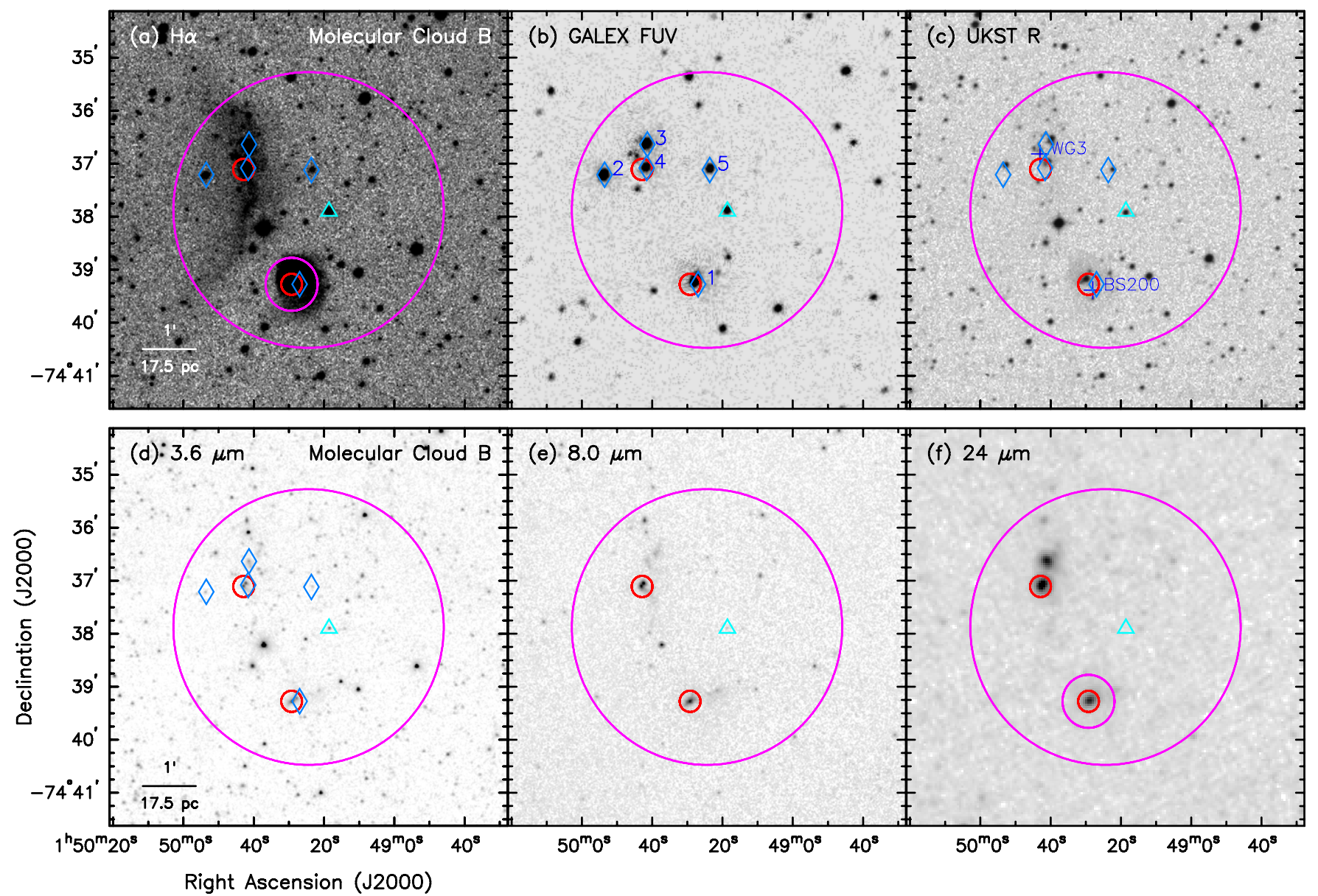

Figure 14. Molecular Cloud B, with the same images and annotations as Figure 13.

(A color version of this figure is available in the online journal.)

and within the arc filament, respectively, and have FUV $=14.33$ and 14.51 and (FUV - NUV) $=-0.38$ and -0.34 , consistent with a B1-2V star. This spatial arrangement of UV stars, optical cluster, and $\mathrm{H} \alpha$ arc shown in Figures 14(a)-(c) suggests that the second brightest UV star has formed an asymmetric H II region with bright emission (arc) resulting from its expansion into the denser ISM surrounding the compact cluster WG3. A fraction of the $\mathrm{H} \alpha$ emission in the arc may come from the third brightest UV star, apparently a member of WG3, but unlikely from other cluster members since they are much fainter in the $\mathrm{UV}$ and hence less massive. Thus, the H II regions in Cloud B, like in Cloud A, are ionized by single or double B2 or B1 stars each with stellar mass $\sim 10$, up to $13 M_{\odot}$.

The current massive star formation in Molecular Cloud B is represented by two embedded YSOs, J014942.43-743704.76 and J014929.21-743916.48, and a HAeBe candidate, J014918.59-743753.93. Both YSOs have mass estimates $\sim 8 M_{\odot}$ (Table 3), and both are in compact $\left(\lesssim 5^{\prime \prime}\right.$, or $\left.\sim 1.3 \mathrm{pc}\right)$ multiple systems that have massive stars of similar masses at different evolutionary stages. As shown in Figure 14, YSO J014942.43-743704.76, dominating the MIR light, is young with an estimated age $\sim 0.1 \mathrm{Myr}$ (Table 3), while the companion UV bright star is the ionizing star of the compact $\mathrm{H}_{\mathrm{II}}$ region and hence at least a few Myr old. The other YSO J014929.21-743916.48 has an estimated age $\sim 0.1 \mathrm{Myr}$, and the companion is a non-embedded, UV bright star with FUV = 15.51 and $(\mathrm{FUV}-\mathrm{NUV})=0.10$, comparable to a B3V but with a color redder by $\sim 0.3 \mathrm{mag}$. Note that this redder color could re- sult from unresolved redder stars in the vicinity, but it would not be caused by extinction since the NUV filter overlaps the $2200 \AA$ absorption peak such that $E(\mathrm{FUV}-\mathrm{NUV}) / A_{V}=-0.17$, making an extincted source fainter and bluer (Cardelli et al. 1989). Lastly, the $\mathrm{HAeBe}$ candidate has estimated mass $\sim 11 M_{\odot}$ and age $\sim 1.0$ Myr (Table 5), falling between the ages of MIRselected YSOs and UV-bright ionizing stars.

Our examination of massive stellar and YSO populations in Molecular Cloud B shows that a few small groups of early B (B1-3) stars formed 3-10 Myr ago. YSO $\mathrm{J} 014929.21-743916.48$ is found in the $\mathrm{H} \alpha$ arc, possibly triggered by expansion of the older arc-shaped H II region. Comparisons in their masses demonstrate the trend generally seen in triggered star formation, i.e., the most massive star formed in the current generation, $\sim 8 M_{\odot}$, is less massive than $\sim 13 M_{\odot}$ in the previous generation. In contrast, the other YSO J014942.43-743704.76 is located in the CO peak of Cloud B revealed by ASTE observations, in which star formation just began in the last few Myr (as evidenced by the bright compact H II region). Given that Cloud B has higher mass of $3 \times 10^{3} M_{\odot}$ (Mizuno et al. 2006), it is possible that the compact system around YSO J014942.43-743704.76 can eventually form a more massive cluster.

\subsubsection{Molecular Cloud C}

Molecular Cloud $\mathrm{C}$ appears to be located along a string of active star formation: from the compact blue cluster NGC 796 at $55^{\prime \prime}(\sim 15 \mathrm{pc})$ north to the cloud, to a large association BS217 


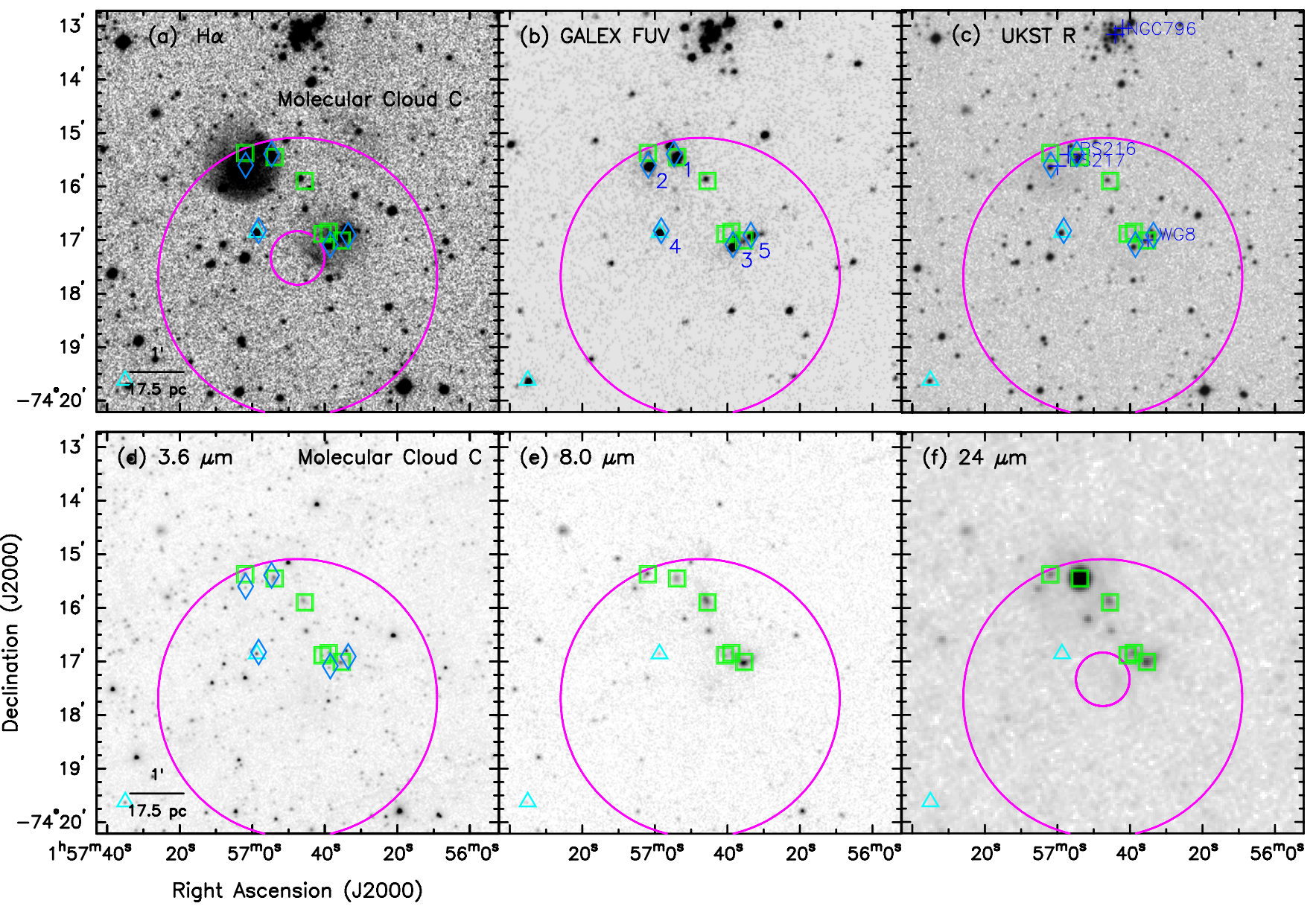

Figure 15. Molecular Cloud C, with the same images and annotations as Figure 13.

(A color version of this figure is available in the online journal.)

containing a compact cluster BS216 in the northern part of the cloud, to a small association WG8 near the center of the cloud (Figure 15(c)). Four $\mathrm{H} \alpha$ blobs have been identified within and north of Cloud C (Muller \& Parker 2007). The north blob has little diffuse emission and is spatially coincident with stars in NGC 796 (Figures 15(a)-(c)); indeed, the spectroscopic study by Ahumada et al. (2002) revealed a small equivalent width (3.89 $\AA$ ) of $\mathrm{H} \alpha$ emission and suggested an age of $\gtrsim 6-10 \mathrm{Myr}$. The other three $\mathrm{H} \alpha$ blobs appear to be typical H II regions. The bright compact H II region at the northeast of the cloud, associated with BS216, contains the two brightest UV stars in the cloud; these stars have FUV $=14.20$ and 14.48 and $(\mathrm{FUV}-\mathrm{NUV})=-0.10$ and -0.27 , respectively, consistent with $\mathrm{B} 1-2 \mathrm{~V}$ stars. The second brightest compact $\mathrm{H}$ II region in the south near the cloud center, associated with WG8, contains the next two UV-brightest stars; they have FUV $=14.83$ and 15.57, and (FUV - NUV) $=-0.11$ and -0.10 , respectively, consistent with $\sim \mathrm{B} 2 \mathrm{~V}$ stars. Finally, the very faint $\mathrm{H}$ II region at the northwest of the cloud appears to center on a blue star of $\mathrm{FUV}=18.10$ and $(\mathrm{FUV}-\mathrm{NUV})=-0.07$, consistent with a B6V star. These three H II regions are ionized by small groups of early B stars or a single mid B star.

Despite the string of active star formation, there is no embedded MIR-bright YSO, but there is a group of six fainter YSO candidates found in Molecular Cloud C (Figure 15). As discussed in Section 3.2, these six candidates are most likely bona fide YSOs based on an examination of their multiwavelength SEDs and images, and further confirmed by IRS spectra of the two with the brightest $24 \mu \mathrm{m}$ emission among them, J015635.59-741701.10 and J015654.01-741526.13. All six YSOs are found within or near the edge of the two bright $\mathrm{H}$ II regions: three in the south-central H II region, two in the northeast $\mathrm{H}$ II region and one near its edge (Figure 15(a)). The three YSOs in south-central $\mathrm{H}$ II region have lower masses, two $\sim 4-5 M_{\odot}$ and one $\sim 8 M_{\odot}$; this group has younger ages with two $\lesssim 0.1$ Myr and one 2 Myr. The other three YSOs in and near the northeast $\mathrm{H}$ II region have higher masses, all three $\sim 7-11 \mathrm{M}_{\odot}$, including the two most massive ones among the fainter YSO candidates; this group has older ages with one $\gtrsim 0.1 \mathrm{Myr}$ and two $~ 3$ Myr (Table 4).

Compared to other Bridge clouds, star formation around Cloud C appears more compactly clustered during the last $10 \mathrm{Myr}$, i.e., from the older cluster NGC 796 to young clusters of UV stars and YSOs found in two compact bright $\mathrm{H}$ II regions. A clustered mode of massive star formation is usually found in high-mass molecular clouds; Cloud $\mathrm{C}$ does provide a preferred environment owing to its high mass, i.e., $7 \times 10^{3} M_{\odot}$, which is the highest among all eight clouds (Mizuno et al. 2006), as well as its location in a region with the highest $N(\mathrm{H} \mathrm{I})$ in the Bridge. There appears to be a sequence of star formation from north to south, i.e., from the $\sim 10$-Myr-old NGC 796, to the south-central $\mathrm{H}$ II region containing two YSOs $\lesssim 0.1 \mathrm{Myr}$. Although at present this south-central H II region has UV stars and YSOs with the lowest masses among the three clusters, it is near the $\mathrm{CO}$ peak of Cloud $\mathrm{C}$ revealed by ASTE observations and may possibly develop a massive cluster similar to its northern neighbors. 

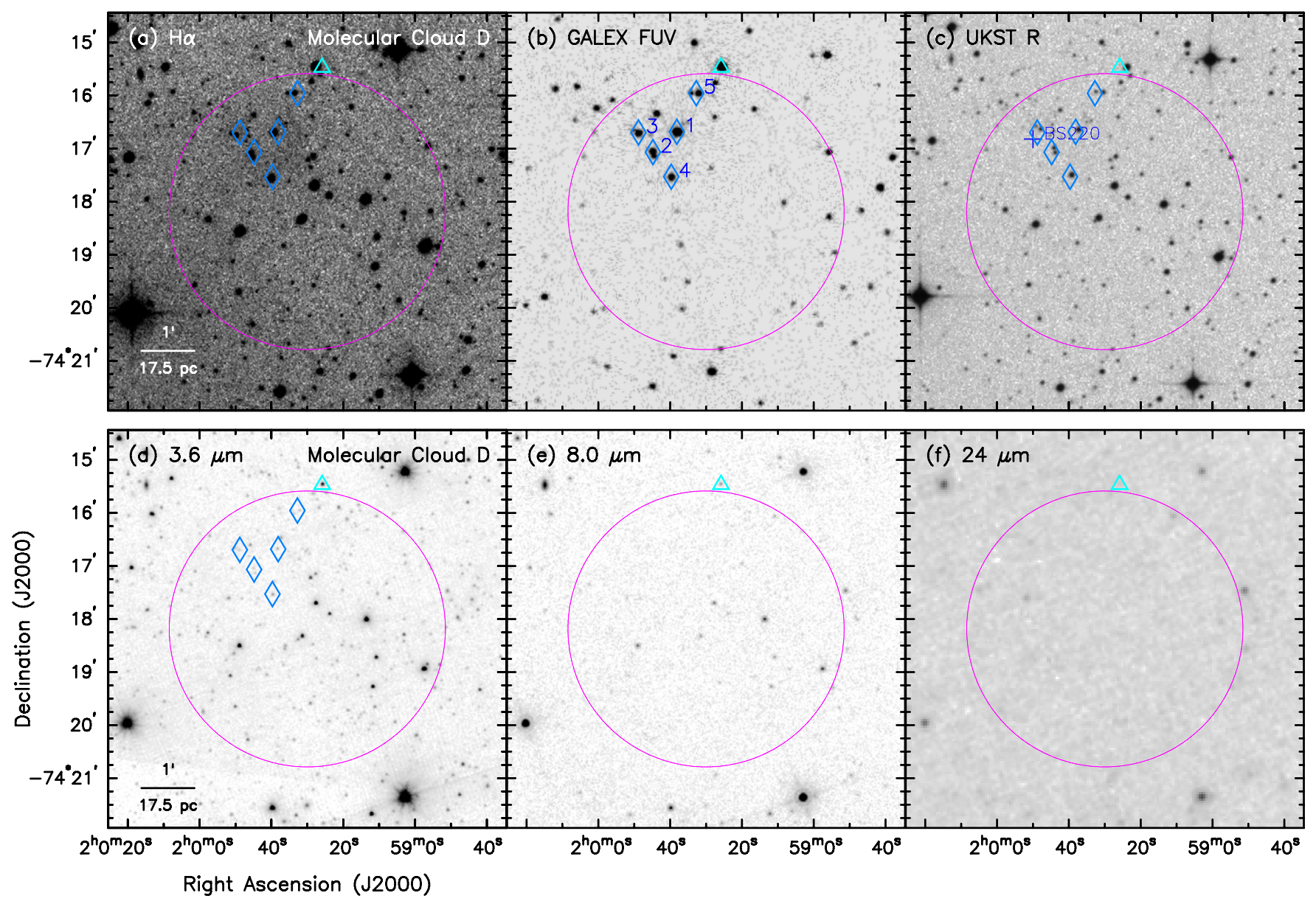

Figure 16. Molecular Cloud D, with the same images and annotations as Figure 13.

(A color version of this figure is available in the online journal.)

\subsubsection{Molecular Cloud D}

Molecular Cloud D overlaps with a stellar association BS220 (Figure 16) and shows no sign of massive star formation in the last $10 \mathrm{Myr}$ due to the lack of $\mathrm{H}$ II regions. Examination on GALEX UV and Spitzer MIR data finds neither blue stars nor YSOs in Cloud D. This cloud appears to be quiescent in star formation, even though its mass, $1 \times 10^{3} M_{\odot}$, is comparable to other clouds in the Bridge (Mizuno et al. 2006). It is possible that Cloud D is just formed and star formation has not yet begun. Alternatively, it is also possibly a false $\mathrm{CO}$ detection since it has the lowest peak main-beam temperature, $10 \mathrm{mK}$, barely higher than the rms noise temperature of $9 \mathrm{mK}$ (Mizuno et al. 2006). Deep CO observations of Cloud D are needed to verify the detection.

\subsubsection{Molecular Cloud E}

Three large stellar associations are located to the east of Molecular Cloud E, with two of them, BD11 and BD13 (Bica $\&$ Dutra 2000), overlapping on the Cloud's north and east edge (Figure 17(c)). Evidence of fairly recent (5-10 Myr old) star formation is shown by the H II shell DEM S 171 that abuts the east side of Cloud E. DEM S 171 is one of the few large $\mathrm{H} \alpha$ structures in the Bridge (Meaburn 1986; Muller \& Parker 2007), with part of the shell rims shown in Figure 17. It has a size of $\sim 8^{\prime} \times 8^{\prime}$, or $130 \times 130 \mathrm{pc}^{2}$, and the shell expansion is suggested to be driven by winds of a Wolf-Rayet star or supernova explosion (Graham et al. 2001). Compared to the plausible Wolf-Rayet star responsible for DEM S 171, the brightest and bluest UV stars in Cloud $\mathrm{E}$ have FUV $=15.64$, and 15.90, and $(\mathrm{FUV}-\mathrm{NUV})=$ -0.14 and -0.32 , respectively, consistent with B2-3V stars. These two stars show no identifiable $\mathrm{H}$ II regions but only some faint diffuse $\mathrm{H} \alpha$ emission in their surroundings (Figure 17(a)), suggesting that they have dispersed their $\mathrm{H}_{\mathrm{II}}$ regions and are relatively old.

Current star formation in Molecular Cloud E is represented by one embedded YSO and two fainter YSOs (Figure 17). The three YSOs, J020649.68-744359.95, J020622.18-744254.43, and J020631.11-744247.84, have mass estimates $\sim 5-6 M_{\odot}$ (Tables 3 and 4), not as massive as the $\sim 8-10 M_{\odot}$ found in other molecular clouds in the Bridge. YSO J020649.68-744359.95 is resolved into a compact multiple that is most clearly visible in the $B$-band image. This compact multiple consists of only embedded sources, unlike YSOs in Clouds A-C that are frequently found in pairs with UV-bright early B stars. YSOs in Cloud E are all found near diffuse $\mathrm{H} \alpha$ emission. YSO J020649.68-744359.95 and fainter YSO J020631.11-744247.84 are located at the edges of the HII shell DEM S 171, and so is the peak of the ASTE CO emission (Figure 17(a)); their formation might have been triggered by the shell expansion. The remaining fainter YSO $\mathrm{J} 020622.18-744254.43$ is near the edge of faint diffuse $\mathrm{H} \alpha$ emission, though it is difficult to distinguish if this diffuse emission is associated with the UV-bright star or from the PSF of the nearby foreground star that is saturated in the $R$-band but not detected in UV images (Figure 17). 


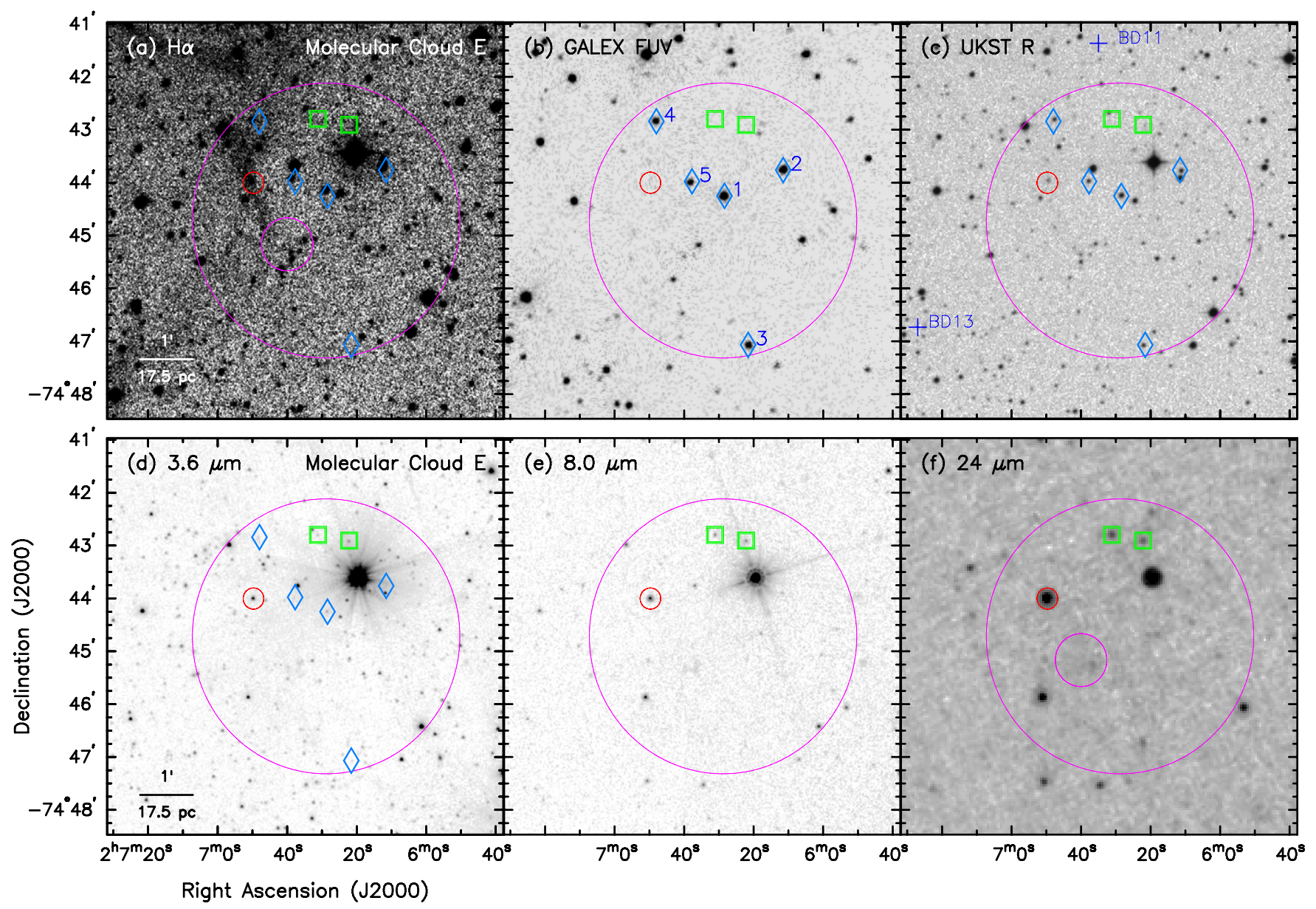

Figure 17. Molecular Cloud E, with the same images and annotations as Figure 13.

(A color version of this figure is available in the online journal.)

Our examination of massive star and YSO populations in Molecular Cloud E shows that a sparse distribution of a few early-to-mid B (B2-3 and later) stars have formed in the recent past $\gtrsim 10 \mathrm{Myr}$, as well as a few more recently. Given that YSOs are on the edge of the H II shells and not as massive as the ionizing star, the current star formation is consistent with a triggered star formation scenario. In addition, these YSOs are the dominant sources in their groups, in contrast to pairs of high-mass stars and YSOs found in other clouds, suggesting that massive star formation may not have been active in Cloud $\mathrm{E}$ until triggered by shell expansion into the molecular cloud or gas accumulation in the shell (e.g., the collect and collapse scenario, Elmegreen 1998). Given Cloud E's low mass of $1 \times 10^{3} M_{\odot}$ (Mizuno et al. 2006) and spread-out distribution of YSOs along the shell rims, the star formation will most likely only ever amount to distributed small groups of early-to-mid B stars.

\subsubsection{Molecular Cloud F}

Molecular Cloud F appears to be in a relatively quiescent environment as there are no cataloged stellar associations or clusters. Evidence of relatively recent massive stars is shown by diffuse, faint $\mathrm{H} \alpha$ emission within the cloud in the $\mathrm{H} \alpha$ image (Figure 18(a)). This H $\alpha$ emission surrounds three bright blue stars revealed by GALEX UV images (Figure 18(b)). They have $\mathrm{FUV}=14.50,14.51$, and 14.53, and $(\mathrm{FUV}-\mathrm{NUV})=-0.16$, -0.11 , and -0.14 , respectively, consistent with B1-2V stars. Some or all three blue stars are likely the ionizing sources this diffuse, low-brightness $\mathrm{H} \alpha$ emission, and they would be $\gtrsim 10$ Myr old.

The current star formation in Cloud $\mathrm{F}$ is represented by one embedded YSO and one fainter YSO (Figure 18). YSO $\mathrm{J} 021440.18-742127.03$ has an estimated mass $\gtrsim 10 M_{\odot}$, among the most massive YSOs in the Bridge (Table 3). It is in a compact system of massive stars at different evolutionary stages, with the other massive source being the second brightest UV star $\sim \mathrm{B} 1-2 \mathrm{~V}$, corresponding to $\sim 13-10 M_{\odot}$. The fainter YSO J021434.94-742339.94 has a mass of $\sim 4 M_{\odot}$ (Table 4$)$ and appears as a single source.

The examination of massive star and YSO populations in Molecular Cloud F shows that distributed early B (B1-2) stars have formed in the recent past ( $\gtrsim 10 \mathrm{Myr}$ ) as well as in the current. There is no dense clustering of higher mass stars/YSOs, not unexpected from Cloud F's low mass of $1 \times 10^{3} M_{\odot}$ (Mizuno et al. 2006). The formation of fainter YSO J021434.94-742339.94 might have been affected by the stellar energy feedback as it is within the diffuse, low-brightness $\mathrm{H} \alpha$ emission, though higher resolution of $\mathrm{H} \alpha$ and $\mathrm{CO}$ maps are needed to assess such a possibility.

\subsubsection{Molecular Clouds $H+G$}

Molecular Cloud $\mathrm{H}$ is part of Cloud $\mathrm{G}$ and hence included in its discussion. Molecular Cloud $\mathrm{G}$ abuts two stellar associations, a large one BD33 on the west and a small one WG18 on the north east. The $\mathrm{H} \alpha$ image shows diffuse, low-brightness emission 

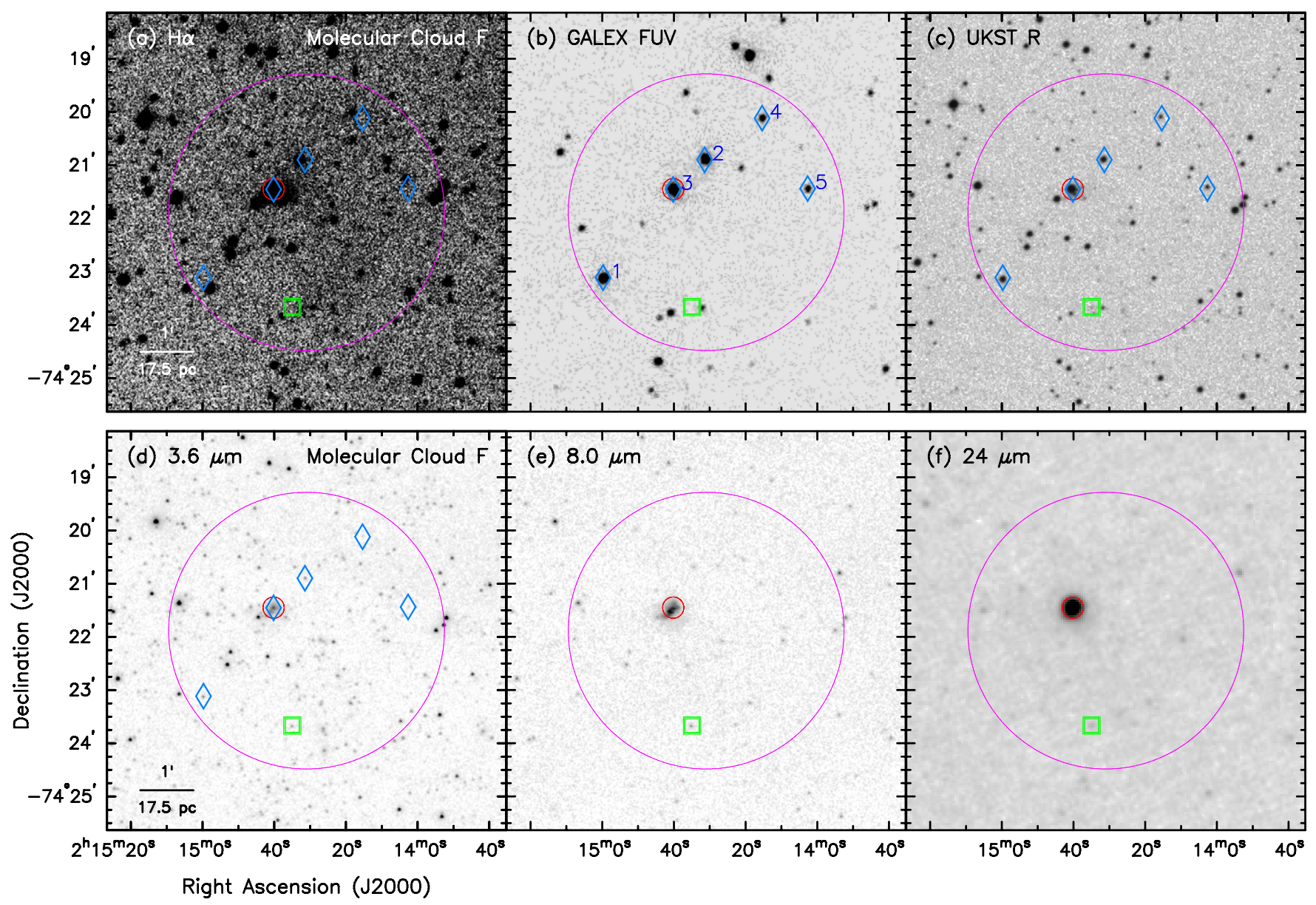

Figure 18. Molecular Cloud F, with the same images and annotations as Figure 13.

(A color version of this figure is available in the online journal.)

within the cloud and slightly brighter emission to the southeast (Figure 19). The two brightest blue stars in the cloud revealed by $G A L E X$ have FUV $=16.28$ and 16.94 , and $(\mathrm{FUV}-\mathrm{NUV})=$ -0.01 and 0.03 , respectively, consistent with $\sim \mathrm{B} 3 \mathrm{~V}$ stars. They are plausibly the ionizing sources of this diffuse $\mathrm{H} \alpha$ emission and would be $\gtrsim 10$ Myr old.

The current star formation in Cloud $G$ is revealed by one embedded YSO and one fainter YSO (Figure 19). YSO J021526.72-740432.77 has an estimated mass $\sim 9 M_{\odot}$ and an age $\leqslant 0.1 \mathrm{Myr}$ (Table 3). It is a single isolated source, unlike the majority of YSOs in other clouds are found in pairs with UV-bright early B stars; the massive star formation in Cloud $\mathrm{G}$ appears to be just beginning. The fainter YSO $\mathrm{J} 021525.25-740600.82$ has a mass $\sim 5 M_{\odot}$. This YSO is within the diffuse faint $\mathrm{H} \alpha$ emission, but association is unclear.

The massive star and YSO contents of Molecular Cloud G show that distributed early-to-mid B $(\sim \mathrm{B} 3)$ stars have formed in the recent past $\gtrsim 10 \mathrm{Myr}$ as well as are currently forming. Although at present there is only one $\sim 9 M_{\odot}$ YSO, it is possibly the first newly formed massive star in a cluster as it is at the ASTE CO peak and Cloud $G$ has the second highest mass of $5 \times 10^{3} M_{\odot}$ in the Bridge (Mizuno et al. 2006). Massive star formation appears to be just beginning in Cloud $\mathrm{G}$.

\subsection{Star Formation Efficiency and Rate}

The relation between gas surface density and SFR is one of the most critical links between star formation and galaxy evolution, and also the most widely used relation in extragalactic astronomy. The observed relation, the "Schmidt-Kennicutt (S-K) law," is tight when properties are averaged on kpc scales (Kennicutt 1989, 1998; Kennicutt et al. 2009), but appears to break down at scales $\leqslant$ a few hundred pc, as shown in recent high-resolution studies of M33 (Onodera et al. 2010; Schruba et al. 2010). It is conceivable that the SFR and GMC content averaged over too small a surface area do not adequately sample GMCs at different evolutionary stages, and thus do not show a good relation. As demonstrated in our LMC study, while individual GMCs show different evolutionary stages (based on their association with different advancement of star formation activities) and have different SFRs, the S-K relation is observed when averaging over several GMCs in large $\mathrm{H}$ II complexes of sizes $\sim 200 \mathrm{pc}$ (Chen et al. 2010). On the other hand, the SFR of a GMC also depends on SFE. Much lower SFRs than expected from the $\mathrm{S}-\mathrm{K}$ relation have been observed in the outer disks of spiral and dwarf galaxies, implying that star formation may be affected by environmental factors (Bigiel et al. 2010). The proximity of the Bridge provides an excellent laboratory to use resolved stellar and gas contents to critically examine environmental effects, specifically metallicity and tidal effects, on star formation.

Applying the same method as our study of massive YSOs in LMC H II regions (Chen et al. 2010), we assess the "instantaneous" SFE, SFE using the known massive YSO content. The total mass of the current star formation, $M_{\mathrm{YSO}}^{\text {total }}$, can be estimated using the number of YSOs within different mass bins and assuming a Salpeter's 


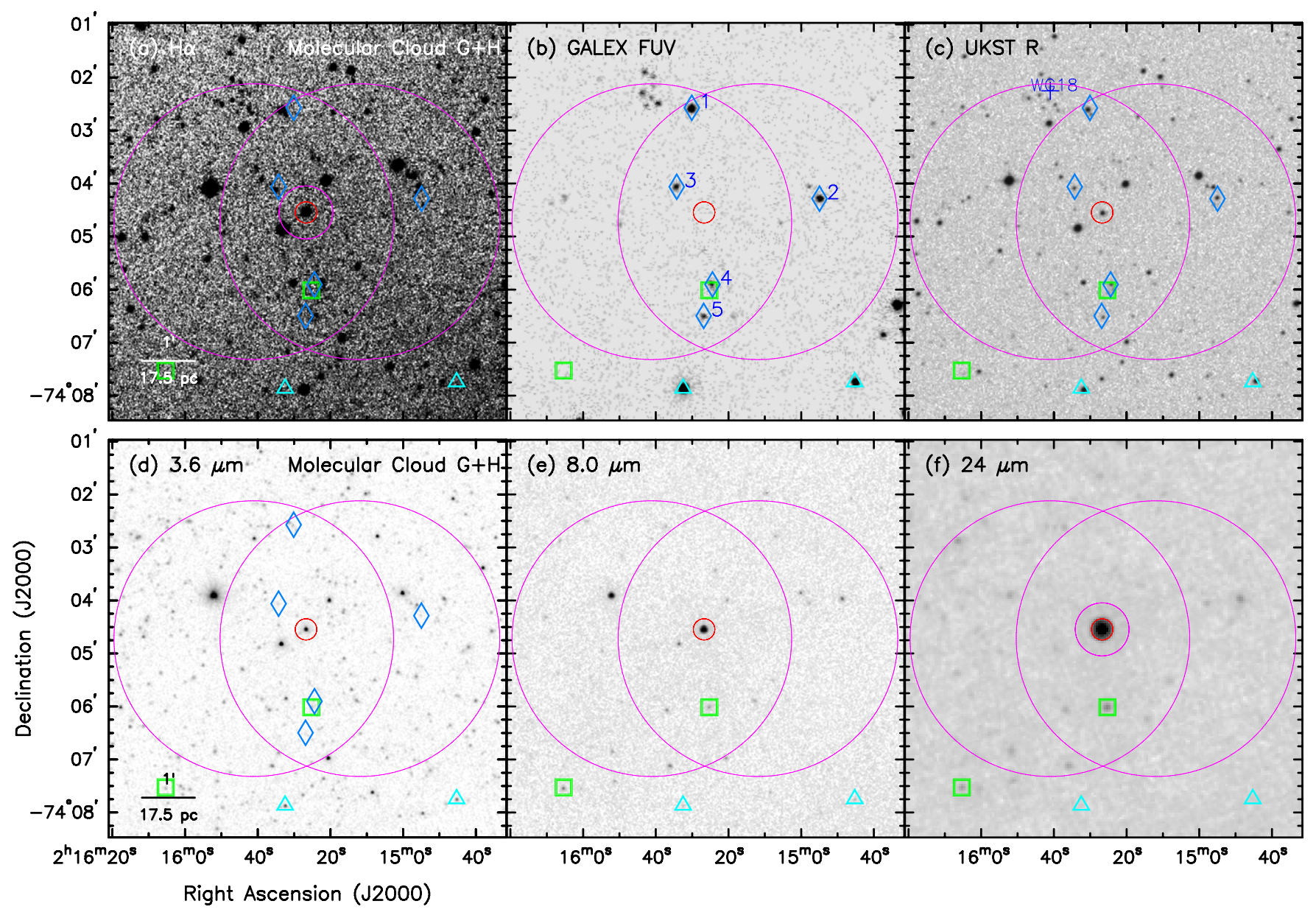

Figure 19. Molecular Cloud G, with the same images and annotations as Figure 13.

(A color version of this figure is available in the online journal.)

stellar initial mass function (IMF). The number of YSOs in individual molecular clouds is too small to infer $M_{\text {YSO }}^{\text {total }}$ with reasonable uncertainties, however, the total number of YSOs in all seven molecular clouds can be used to assess an average $\mathrm{SFE}_{\mathrm{YSO}}$ for the collection of clouds, albeit averaging over different evolutionary stages (recall Section 5.2). $M_{\text {YSO }}^{\text {total }}$ of the seven molecular clouds is calculated by integrating the IMF from lower to upper mass limits, $M_{l}$ and $M_{u}$. We use the highest mass of YSO observed for $M_{u}$ and $1 M_{\odot}$ for $M_{l}$; the adoption of $1 M_{\odot}$ is to facilitate comparisons with other work as it is commonly used. $M_{\text {YSO }}^{\text {total }}$ is estimated excluding fainter YSOs, since except for the two confirmed by IRS spectra, their identification as YSOs is less certain. The $M_{\mathrm{YSO}}^{\text {total }}$ is then divided by the sum of cloud masses to obtain $\mathrm{SFE}_{\mathrm{YSO}}$, and these three quantities are listed in Table 6. Also listed are uncertainties in $M_{\text {YSO }}^{\text {total }}$; they are directly related to the uncertainties in mass estimates for individual massive YSOs used in number counts. Such uncertainties are thus estimated using the largest and smallest mass ranges covered by these YSOs. This exercise yields a dimensionless efficiency (mass ratio) of $0.015_{-0.004}^{+0.009}$.

To determine the current star formation rate, $\mathrm{SFR}_{\mathrm{YSO}}$, from $M_{\mathrm{YSO}}^{\text {total }}$ requires a timescale. While the age of each massive YSO can be constrained using its evolutionary stage, uncertainties of high-mass YSO accretion models make it difficult to set this age more precisely than $\lesssim 1 \mathrm{Myr}$. On the other hand, if we presume that all high- and intermediate-mass YSOs within molecular clouds are currently forming in a burst, the formation timescale
Table 6

Star Formation Properties in the Bridge

\begin{tabular}{lcc}
\hline \hline Property & Molecular Clouds & Whole Area \\
\hline Size & $7 \times 2.6$-radius circles & $180^{\prime} \times 80^{\prime}$ \\
Area $\left(\mathrm{kpc}^{2}\right)$ & 0.038 & 3.4 \\
$M_{\mathrm{CO}}\left(10^{3} M_{\odot}\right)$ & 19 & $\ldots$ \\
$N_{\mathrm{YSO}}\left(M_{\mathrm{u} 1}-M_{\mathrm{u} 2}\right)^{\mathrm{a}}$ & $6(10.4-6.1)$ & $21(10.4-6.0)$ \\
$M_{\mathrm{YSO}}^{\text {total }}\left(M_{\mathrm{u}}-M_{1}\right)^{\mathrm{b}}\left(M_{\odot}\right)$ & $290_{-70}^{+170}(10.4-1)$ & $970_{-315}^{+630}(10.4-1)$ \\
$\mathrm{SFE}_{\mathrm{YSO}}$ & $0.015_{-0.004}^{+0.009}$ & $\ldots$ \\
$\mathrm{SFR}_{\mathrm{YSO}}\left(M_{\odot} \mathrm{yr}^{-1}\right)$ & $2.9 \mathrm{E}-4$ & $9.7 \mathrm{E}-4$ \\
$\Sigma_{\mathrm{SFRYSO}}\left(M_{\odot} \mathrm{yr}^{-1} \mathrm{kpc}^{-2}\right)$ & $7.6_{-1.8}^{+4.5} \mathrm{E}-3$ & $2.8_{-0.9}^{+1.9} \mathrm{E}-4$ \\
$\log _{(}\left(24 \mu \mathrm{m}^{2}\left[\mathrm{ergs} \mathrm{s}^{-1}\right]\right)$ & 37.5 & 39.0 \\
$\mathrm{SFR}_{24}\left(M_{\odot} \mathrm{yr}^{-1}\right)$ & $2.0 \mathrm{E}-5$ & $4.2 \mathrm{E}-4$ \\
$\Sigma_{\mathrm{SFR} 24}\left(M_{\odot} \mathrm{yr}^{-1} \mathrm{kpc}^{-2}\right)$ & $5.2_{-2.6}^{+2.6} \mathrm{E}-4$ & $1.2_{-0.6}^{+0.6} \mathrm{E}-4$ \\
$\Sigma_{\mathrm{HI}}\left(M_{\odot} \mathrm{pc}^{-2}\right)$ & 17.9 & 10.2 \\
$\Sigma_{\mathrm{H}}\left(M_{\odot} \mathrm{pc}^{-2}\right)$ & 1.6 & $\ldots$ \\
$\Sigma_{\mathrm{SFRGa}}\left(M_{\odot} \mathrm{yr}^{-1} \mathrm{kpc}^{-2}\right)$ & 0.016 & $6.0 \mathrm{E}-3$ \\
\hline
\end{tabular}

Notes.

a Number of YSOs with $\bar{M}_{*}$ in the mass range (u1-u2).

b Total mass of YSOs extrapolated for the mass range $(\mathrm{u}-1)$.

for a cluster or association may be more relevant to the cloud efficiency than the age of any individual protostar. Carrying on with this assumption, we adopt a cluster formation time of $\sim 1 \mathrm{Myr}$ (e.g., Bonnell et al. 2003) and derive SFR YSO $_{\text {of }} 2.9 \times$ $10^{-4} M_{\odot} \mathrm{yr}^{-1}$ and SFR $\mathrm{YSO}$ per surface area, $\Sigma_{\mathrm{SFRYSO}}$, of $7.6 \times$ $10^{-3} M_{\odot} \mathrm{yr}^{-1} \mathrm{kpc}^{-2}$ (Table 6). 
For comparison, we have also estimated the SFR averaged over the last $\sim 10 \mathrm{Myr}$, commonly derived from $\mathrm{H} \alpha$ and/or $24 \mu \mathrm{m}$ fluxes calibrated on an ensemble of $\mathrm{H}$ II regions (e.g., Kennicutt 1998). The average SFR of the Bridge is estimated using the integrated $24 \mu \mathrm{m}$ flux with the prescription of Calzetti et al. (2007):

$$
\operatorname{SFR}_{24}\left(M_{\odot} \mathrm{yr}^{-1}\right)=1.27 \times 10^{-38}[L(24 \mu \mathrm{m})]^{0.8850},
$$

where $L(24 \mu \mathrm{m})$ is the $24 \mu \mathrm{m}$ luminosity in ergs s $\mathrm{s}^{-1}$. To measure the $24 \mu \mathrm{m}$ luminosity, we use an aperture size of 5.2, twice the beam size of the NANTEN CO observations since the molecular clouds were not resolved; the largest uncertainties come from flux calibration, $\sim 10 \%$ (MIPS Data Handbook). The $24 \mu \mathrm{m}$ luminosity, $\mathrm{SFR}_{24}$, and $\mathrm{SFR}_{24}$ per surface area, $\Sigma_{\mathrm{SFR} 24}$, are given in Table 6. The Bridge has $\Sigma_{\mathrm{SFR} 24}=5.2 \times 10^{-4} M_{\odot} \mathrm{yr}^{-1} \mathrm{kpc}^{-2}$, much lower than $\Sigma_{\text {SFRYSO }}=7.6 \times 10^{-3}$ by a factor of 15 . This discrepancy is consistent with our previous analysis of LMC regions, in which we found that below a threshold gas surface density of $\sim 200 M_{\odot} \mathrm{pc}^{-2}$, SFRs derived from detailed YSO analysis frequently exceed the rates calculated from integrated $24 \mu \mathrm{m}$ and $\mathrm{H} \alpha$ emission (Chen et al. 2010; Indebetouw et al. 2008). We attributed this to the ability of detailed YSO analysis to better account for variations in cloud evolutionary state and stellar mass function than the integrated measures.

Within the limitations of the sparse $\mathrm{CO}$ measurements, we can determine where the Bridge clouds lies relative to the S-K relation. The SFR per surface area expected from the $\mathrm{S}-\mathrm{K}$ relation is

$$
\Sigma_{\mathrm{SFRGas}}\left(M_{\odot} \mathrm{yr}^{-1} \mathrm{kpc}^{-2}\right)=2.5 \times 10^{-4}\left(\frac{\Sigma_{\mathrm{Gas}}}{M_{\odot} \mathrm{pc}^{-2}}\right)^{1.4}
$$

where $\Sigma_{\text {Gas }}$ is the sum of molecular and atomic surface densities, $\Sigma_{\mathrm{H} 2}+\Sigma_{\mathrm{HI}}$ (Kennicutt 1998). The average $\Sigma_{\mathrm{H} 2}$ for the Bridge clouds is estimated from the $\mathrm{CO}$ intensities given in Mizuno et al. (2006) and a CO-to- $\mathrm{H}_{2}$ conversion factor $X_{\mathrm{CO}}=1.4 \times$ $10^{21} \mathrm{~cm}^{-2}\left(\mathrm{~K} \mathrm{~km} \mathrm{~s}^{-1}\right)^{-1}$ used in that paper. The average $\Sigma_{\mathrm{H}}$ is measured from Hi maps (Muller et al. 2003a). Then the expected SFR from the total gas surface density is given in Table 6. The largest uncertainties come from the range of $X_{\mathrm{CO}}=1.3_{-0.8}^{+1.6} 10^{21} \mathrm{~cm}^{-2}\left(\mathrm{~K} \mathrm{~km} \mathrm{~s}^{-1}\right)^{-1}$ found in the Bridge (Muller et al. 2010); since $\Sigma_{\mathrm{H} 2}$ comprises 5\%-17\% $\Sigma_{\mathrm{Gas}}$, the associated uncertainty in $\Sigma_{\mathrm{SFRGas}}$ is thus $<20 \%$. As shown in Figure 20 , the Bridge has $\Sigma_{\text {SFRGas }} \sim 31$ times $\Sigma_{\text {SFR } 24}$, while $\Sigma_{\text {SFRYSO }}$ is in agreement with $\Sigma_{\text {SFRGas }}$ within a factor of two. If $\Sigma_{\mathrm{H} 2}$ found in the seven molecular clouds is representative for all clouds associated with YSOs in the Bridge, using the whole sample of YSOs shows a similar trend that SFRs estimated with a comprehensive YSO inventory agree with SFRs expected from the S-K relation and they are much higher than inferred from integrated $24 \mu \mathrm{m}$ luminosities. This discrepancy between $\Sigma_{\mathrm{SFR} 24}$ and $\Sigma_{\text {SFRYSO }}$ is also seen in some GMCs in the LMC, namely, those without bright $\mathrm{H}$ il regions (Indebetouw et al. 2008; Chen et al. 2010). For such GMCs in the LMC, we attributed the derivation of lower $\Sigma_{\mathrm{SFR} 24}$ to their lower luminosity-to-mass $(L / M)$ ratios as the star formation mostly occurred in lowermass or less rich clusters that do not fully sample the high-mass end (with high $L / M$ ) of the stellar IMF, while the prescription in Calzetti et al. (2007, i.e., Equation (1)) is based on rich clusters with high $L / M$. It is not surprising that the Bridge follows the same trend, since its star formation is dominated by small, low-mass clusters evidenced by the scarcity of bright, large H II regions (Muller \& Parker 2007) and spread-out distribution of

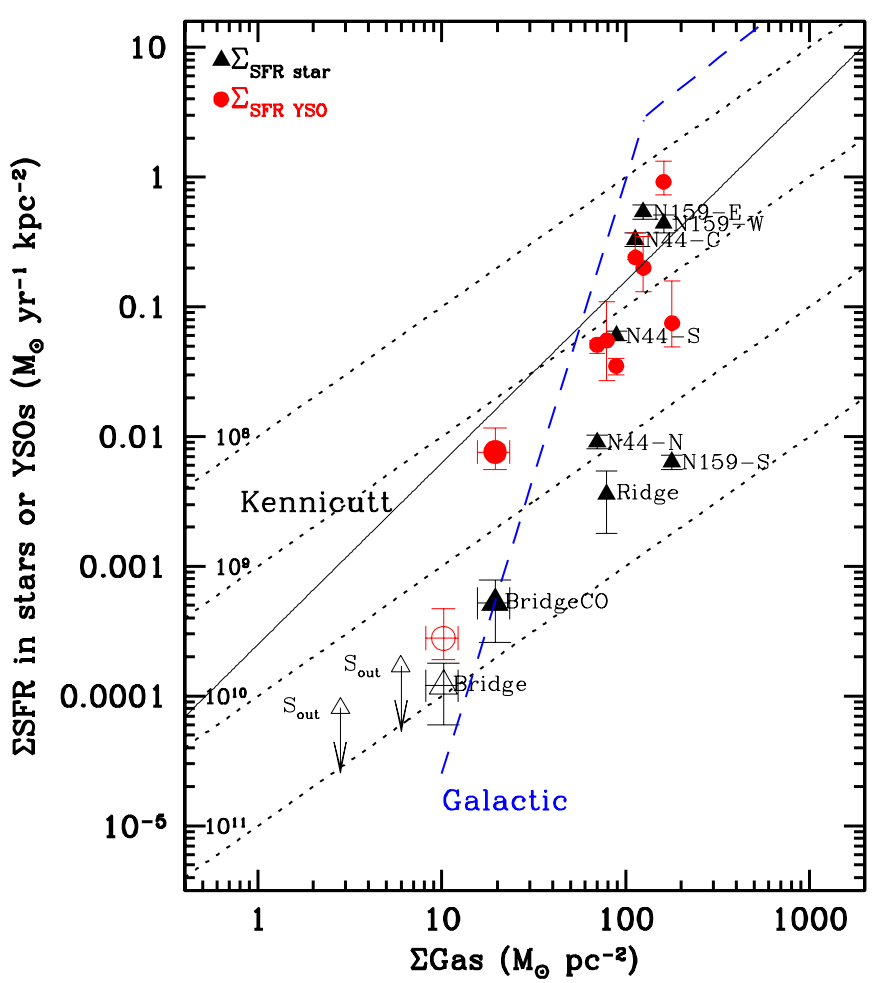

Figure 20. Relation between the SFR per unit area and gas density of all molecular clouds (i.e., locations of $\mathrm{CO} J=1-0$ detection; solid symbols with a label "BridgeCO") and the entire studied area (open symbols with a label "Bridge") in the Bridge. For comparison with the Bridge molecular clouds, also plotted are SFRs estimated for the molecular ridge (Ridge) and six individual GMCs in H II complexes in the LMC (Indebetouw et al. 2008; Chen et al. $2010)$. Each region has two estimated SFRs using different tracers, $\Sigma_{\text {SFRYSO }}$ (circles) and $\Sigma_{\text {SFRstar }}$ (triangles labeled by the region name); in the Bridge, $\Sigma_{\text {SFRstar }}=\Sigma_{\text {SFR24 }}$ while for other regions $\Sigma_{\text {SFRstar }}$ are estimated using integrated $\mathrm{H} \alpha+24 \mu \mathrm{m}$ fluxes. The solid line is the S-K relation, the dotted lines correspond to gas depletion timescales at constant SFRs from $10^{8}$ to $10^{11} \mathrm{yr}$, and the dashed lines mark the broken power law derived from Galactic star-forming regions (Heiderman et al. 2010). GMCs without prominent $\mathrm{H}$ II regions, i.e., the Bridge, Ridge, and two LMC GMCs N 44-N and N 159-S have $\Sigma_{\text {SFRstar }} 11-56$ times smaller than expected from the S-K relation, but their $\Sigma_{\text {SFRYSO }}$ are in better agreement with the S-K relation. Finally, the SFR estimated with FUV and $N(\mathrm{H}$ I) for the outer disks of 17 nearby spiral galaxies (Bigiel et al. 2010) are plotted and labeled as $\mathrm{S}_{\text {out }}$.

(A color version of this figure is available in the online journal.)

intermediate- and high-mass YSOs. It is worth noting that at lower $\Sigma_{\text {Gas }}, \Sigma_{\text {SFRYSO }}$ of molecular clouds in the Bridge is still in agreement with the S-K relation.

Finally, we examine current star formation and estimate SFE and SFR for the Bridge as a whole. The total mass of current star formation can be estimated using the YSO content. The lowest mass bin used for counting depends on the photometric completeness of infrared sources in the Bridge. We have constructed a mass function of the YSOs and found moderate incompleteness at masses $<6 M_{\odot}$ (Figure $8(\mathrm{a})$ ); thus, only masses $\geqslant 6 M_{\odot}$ are used in the counting. $M_{\mathrm{YSO}}^{\text {total }}, \mathrm{SFR}_{\mathrm{YSO}}$, and $\Sigma_{\text {SFRYSO }}$ are estimated for the entire Bridge and listed in Table 6 . For comparison, $\Sigma_{\mathrm{SFR} 24}$ and $\Sigma_{\mathrm{SFRGas}}$ are also estimated using $L(24 \mu \mathrm{m})$ and $\Sigma_{\mathrm{H}_{\mathrm{I}}}$ of the entire Bridge and given in Table 6 . As shown in Figure 20, $\Sigma_{\text {SFRYSO }}$ of the whole Bridge drops by a factor of 27 compared to $\Sigma_{\text {SFRYSO }}$ of all molecular clouds in the Bridge, while there is less than a factor of three difference in the predicted $\Sigma_{\text {SFRGas }}$ from $\Sigma_{\text {Gas }}$ in all clouds and compared to the entire Bridge area. If all YSOs in the Bridge are formed in molecular clouds, this much lower $\Sigma_{\text {SFRYSO }}$ of the whole 
area would indicate a much lower fraction of converting $\mathrm{H}$ I to molecular gas, consistent with that expected for low-metallicity environment (Krumholz et al. 2009). We have further examined if SFRs in molecular clouds in the Bridge are extremely low by comparing them to the $\Sigma_{\mathrm{Gas}}-\Sigma_{\mathrm{SFR}}$ relation derived using resolved YSOs and massive clumps in star-forming regions in the Galaxy (Heiderman et al. 2010). The Heiderman et al. (2010) study demonstrated a much higher $\Sigma_{\mathrm{SFR}}$ than that expected from the $\mathrm{S}-\mathrm{K}$ relation (as can be seen in Figure 20). Despite the very low $\Sigma_{\text {Gas }}$ that is not covered in the Galactic sample, molecular clouds in the Bridge show a higher $\Sigma_{\mathrm{SFR}}$ than expected from the Galactic relation, favoring that the low $\Sigma_{\mathrm{SFR}}$ in the entire Bridge is more likely attributed to a low efficiency in cloud formation.

A similarly low SFR is found in the recent study of the outer disks of spiral and dwarf galaxies, using FUV as SFR tracer and $\mathrm{H}$ I for gas density (Bigiel et al. 2010). For comparison, we have used their data of spiral galaxies to estimate the average

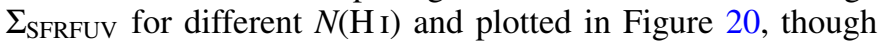
only upper limits can be obtained since data with $\mathrm{S} / \mathrm{N}<3 \sigma$ are not included in their table. These $\Sigma_{\text {SFRFUV }}$ of the outer disks of spirals and $\Sigma_{\text {SFRYSO }}$ of the whole area of the Bridge lie near that expected for long gas depletion time of $\sim 10^{10-11} \mathrm{yr}$. If SFRs in these outer disks have been at similar level in the last $100 \mathrm{Myr}$, their low $\Sigma_{\text {SFRFUV }}$ is likely a result of the inefficiency of molecular cloud formation such as in the Bridge.

Lastly, the YSO content in the Bridge also indicates a possibly different star formation mode. Extrapolating from the total number of YSOs found in the 6-10.4 $M_{\odot}$ (Table 6), the expected number of YSOs with $M_{\star}=11-50 M_{\odot}$ is $15_{-5}^{+11}$ for a Salpeter IMF, and even $7_{-2}^{+5}$ should be O-type $\left(17.5-50 M_{\odot}\right)$ YSOs. However no YSOs with $M_{\star}>10 M_{\odot}$ were found in the Bridge. It is unlikely that higher-mass YSOs are at even earlier evolutionary stages since our FIR Herschel survey of the Bridge show no such embedded high-mass YSOs (Meixner et al. 2013). It is not clear if the higher-mass YSOs are simply not yet formed or such YSOs are less likely to be formed in the Bridge's environment. Alternatively, the total number of YSOs in the $6-10.4 M_{\odot}$ in the Bridge might be overpopulated as a result of preferentially lower-mass star formation expected in the colliding flow scenario (recall Section 5.1). To investigate if star formation mode is different in the Bridge, a more comprehensive census of low-mass YSOs is needed.

\section{SUMMARY}

We have analyzed the distribution of young stellar objects (YSOs) in the Magellanic Bridge, the nearest tidally disturbed low-metallicity system. We selected YSO candidates from Spitzer photometry obtained for the SAGE-SMC Legacy program (Gordon et al. 2009), using a combination of color and SED cuts, building on the techniques of Whitney et al. (2008) and Gruendl \& Chu (2009). We present a list of high-confidence brighter massive YSOs, and a list of somewhat fainter YSO candidates which may suffer from some contamination by unresolved background galaxies. We fit each source's SED with dust radiative transfer models from Robitaille et al. (2006) to constrain physical properties including central mass, envelope mass, and accretion rate. We find only weak correlation between the evolutionary "Stage" implied by the SED fit (high accretion rate normalized to central source mass) and our "Type" classification, in which sources with redder SEDs and fainter parsec-scale interstellar diffuse emission are presumed to be less evolved. The differences are likely due to a combination of a not extremely tight relation between circumstellar environment and protostellar evolutionary stage (which affects the "Type"), and the fact that not all warm circumstellar dust contributing to the FIR SED may actually accrete onto the protostar (which affects the "Stage"). We also analyzed Spitzer photometry for a list of NIR-derived candidate HAeBe stars (Nakajima et al. 2005) in the Bridge and found that about half of the HAeBe candidates show no significant MIR excess and that the other half we modeled as Stage II, or more evolved, YSOs. All of these findings are consistent with our similar analysis of LMC star formation regions.

YSOs in the Bridge do show one particular contrast to those with similar mass in the higher metallicity LMC. Sources at the same evolutionary stage (derived primarily from the FIR flux and derived circumstellar dust mass) are brighter at optical wavelengths in the Bridge compared to the LMC. One explanation is that the circumstellar envelopes are more permeable or clumpy at low metallicity, allowing more shortwavelength radiation to escape for the same mass of warm clumps. Another particularity of the Bridge arises from the comparison of MIR-selected YSOs and NIR-selected HAeBe candidates - the most massive YSOs are less massive than the most massive HAeBe candidates. This is consistent with the star formation in the Bridge becoming less vigorous with time, i.e., forming less massive stars than in the past.

Of particular interest is star formation activity at the locations where CO 1-0 has been detected by Mizuno et al. (2006). All CO 1-0 clouds except their lowest signal-to-noise detection are associated with MIR YSOs. Many of the clouds contain multiple YSOs, and several show evidence of multiple generations of star formation and possible triggering, for example, a large H II region with MIR YSOs on its rim. Many of the YSOs in clouds are also closely associated with UV-bright mainsequence intermediate to massive stars, further supporting heterogeneity and non-coeval formation.

We compared the overall SFR and efficiency in CO-detected molecular clouds to the atomic and molecular gas column. Global measures of star formation (integrated $24 \mu \mathrm{m}$ luminosities) fall below the rates predicted by the total gas column density and the S-K relation. However, detailed analysis of the YSO content brings the measured rates in closer agreement with gasbased predictions - this agrees with results in LMC regions and may be a result of incomplete sampling of the stellar mass function, which systematically reduces integrated $\mathrm{H} \alpha$ and $24 \mu$ because of the steep stellar mass-luminosity relation. When the entire Bridge area is considered (in contrast to only the molecular clouds), the SFR predicted from the relatively high $\mathrm{H}$ I mass is significantly higher than the total star formation that we detect. This is consistent with very inefficient molecular cloud formation efficiency in this environment but relatively efficient star formation in molecular clouds that can form.

This work was supported through JPL grants 1282653 and 1288328 , and also partly by the European Research Council for the ERC Advanced Grant GLOSTAR under contract No. 247078. M.S. acknowledges financial support from the NASA ADAP award NNX11AG50G. M.M. and J.P.S. acknowledge financial support from the NASA Herschel Science Center, JPL contract Nos. 1381522 and 1381650. This study made use of data products of the Two Micron All Sky Survey, which is a joint project of the University of Massachusetts and the Infrared Processing and Analysis Center/California Institute of 
Technology, funded by the National Aeronautics and the Space Administration and the National Science Foundation.

\section{REFERENCES}

Ahumada, A. V., Clariá, J. J., Bica, E., \& Dutra, C. M. 2002, A\&A, 393, 855 Bagheri, G., Cioni, M.-R. L., \& Napiwotzki, R. 2013, A\&A, 551, A78 Beech, M., \& Mitalas, R. 1994, ApJS, 95, 517

Bergvall, N., Laurikainen, E., \& Aalto, S. 2003, A\&A, 405, 31

Besla, G., Kallivayalil, N., Hernquist, L., et al. 2010, ApJL, 721, L97

Bica, E., \& Dutra, C. M. 2000, AJ, 119, 1214

Bica, E. L. D., \& Schmitt, H. R. 1995, ApJS, 101, 41

Bigiel, F., Leroy, A., Walter, F., et al. 2010, AJ, 140, 1194

Bolatto, A. D., Simon, J. D., Stanimirović, S., et al. 2007, ApJ, 655, 212

Bonanos, A. Z., Lennon, D. J., Köhlinger, F., et al. 2010, AJ, 140, 416

Bonnell, I. A., Bate, M. R., \& Vine, S. G. 2003, MNRAS, 343, 413

Boyer, M. L., Srinivasan, S., van Loon, J. T., et al. 2011, AJ, 142, 103

Brott, I., \& Hauschildt, P. H. 2005, in The Three-Dimensional Universe with Gaia, ed. C. Turon, K. S. O'Flaherty, \& M. A. C. Perryman (ESA SP-576; Noordwijk: ESA), 565

Calzetti, D., Kennicutt, R. C., Engelbracht, C. W., et al. 2007, ApJ, 666, 870

Cardelli, J. A., Clayton, G. C., \& Mathis, J. S. 1989, ApJ, 345, 245

Castelli, F., \& Kurucz, R. L. 2004, arXiv:astro-ph/0405087

Chen, C.-H. R., Chu, Y.-H., Gruendl, R. A., Gordon, K. D., \& Heitsch, F. 2009, ApJ, 695, 511

Chen, C.-H. R., Indebetouw, R., Chu, Y.-H., et al. 2010, ApJ, 721, 1206

Chu, Y.-H., Gruendl, R. A., Chen, C.-H. R., et al. 2005, ApJL, 634, L189

Cioni, M.-R. L., Clementini, G., Girardi, L., et al. 2011, A\&A, 527, A116

Dame, T. M. 1993, in AIP Conf. Proc. 278, Back to the Galaxy, ed. S. S. Holt \& F. Verter (Melville, NY: AIP), 267

Demers, S., \& Battinelli, P. 1998, AJ, 115, 154

Demers, S., \& Irwin, M. J. 1991, A\&AS, 91, 171

de Wit, W. J., Beaulieu, J. P., Lamers, H. J. G. L. M., Coutures, C., \& Meeus, G. 2005, A\&A, 432, 619

de Wit, W. J., Beaulieu, J.-P., Lamers, H. J. G. L. M., Lesquoy, E., \& Marquette, J.-B. 2003, A\&A, 410, 199

Draine, B. T., \& Li, A. 2007, ApJ, 657, 810

Elmegreen, B. G. 1998, in ASP Conf. Ser. 148, Origins, ed. C. E. Woodward, J. M. Shull, \& H. A. Thronson, Jr. (San Francisco, CA: ASP), 150

Elmegreen, B. G., \& Efremov, Y. N. 1997, ApJ, 480, 235

Fleener, C. E., Payne, J. T., Chu, Y., Chen, C., \& Gruendl, R. A. 2010, AJ, 139,158

Franceschini, A., Manners, J., Polletta, M. d. C., et al. 2005, AJ, 129, 2074

Gardiner, L. T., Sawa, T., \& Fujimoto, M. 1994, MNRAS, 266, 567

Gordon, K. D., Bot, C., Muller, E., et al. 2009, ApJL, 690, L76

Gordon, K. D., Meixner, M., Meade, M. R., et al. 2011, AJ, 142, 102

Graham, M. F., Smith, R. J., Meaburn, J., \& Bryce, M. 2001, MNRAS, 326, 539

Grebel, E. K. 1997, A\&A, 317, 448

Groenewegen, M. A. T. 2006, A\&A, 448, 181

Gruendl, R. A., \& Chu, Y. 2009, ApJS, 184, 172

Hambly, N. C., Irwin, M. J., \& MacGillivray, H. T. 2001a, MNRAS, 326,1295

Hambly, N. C., MacGillivray, H. T., Read, M. A., et al. 2001b, MNRAS, 326,1279

Harries, T. J., Hilditch, R. W., \& Howarth, I. D. 2003, MNRAS, 339, 157

Harris, J. 2007, ApJ, 658, 345

Hartmann, L., Ballesteros-Paredes, J., \& Bergin, E. A. 2001, ApJ, 562, 852

Harvey, P. M., Chapman, N., Lai, S.-P., et al. 2006, ApJ, 644, 307

Hatziminaoglou, E., Pérez-Fournon, I., Polletta, M., et al. 2005, AJ, 129, 1198

Heiderman, A., Evans, N. J., II, Allen, L. E., Huard, T., \& Heyer, M. 2010, ApJ, 723, 1019

Heitsch, F., Slyz, A. D., Devriendt, J. E. G., Hartmann, L. W., \& Burkert, A. 2006, АpJ, 648, 1052
Hillenbrand, L. A., Strom, S. E., Vrba, F. J., \& Keene, J. 1992, ApJ, 397, 613 Hindman, J. V., Kerr, F. J., \& McGee, R. X. 1963, AuJPh, 16, 570

Indebetouw, R., Whitney, B. A., Johnson, K. E., \& Wood, K. 2006, ApJ, 636,362

Indebetouw, R., Whitney, B. A., Kawamura, A., et al. 2008, AJ, 136, 1442

Irwin, M. J., Demers, S., \& Kunkel, W. E. 1990, AJ, 99, 191

Kato, D., Nagashima, C., Nagayama, T., et al. 2007, PASJ, 59, 615

Kennicutt, R. C., Hao, C.-N., Calzetti, D., et al. 2009, ApJ, 703, 1672

Kennicutt, R. C., Jr. 1989, ApJ, 344, 685

Kennicutt, R. C., Jr. 1998, ApJ, 498, 541

Keto, E. 2007, ApJ, 666, 976

Kim, S., Staveley-Smith, L., Dopita, M. A., et al. 2003, ApJS, 148, 473

Krumholz, M. R., McKee, C. F., \& Tumlinson, J. 2009, ApJ, 693, 216

Larson, R. B., \& Tinsley, B. M. 1978, ApJ, 219, 46

Lee, J., Rolleston, W. R. J., Dufton, P. L., \& Ryans, R. S. I. 2005, A\&A, 429,1025

Leroy, A., Bolatto, A., Stanimirovic, S., et al. 2007, ApJ, 658, 1027

Leroy, A. K., Bolatto, A., Bot, C., et al. 2009, ApJ, 702, 352

Li, A., \& Draine, B. T. 2001, ApJ, 554, 778

Mac Low, M.-M., \& Ferrara, A. 1999, ApJ, 513, 142

Meaburn, J. 1986, MNRAS, 223, 317

Meixner, M., Panuzzo, P., Roman-Duval, J., et al. 2013, AJ, 146, 62

Mizuno, N., Muller, E., Maeda, H., et al. 2006, ApJL, 643, L107

Muller, E., Mizuno, N., Minamidani, T., et al. 2014, PASJ, 66, 4

Muller, E., Ott, J., Hughes, A., et al. 2010, ApJ, 712, 1248

Muller, E., \& Parker, Q. A. 2007, PASA, 24, 69

Muller, E., Staveley-Smith, L., Zealey, W., \& Stanimirović, S. 2003a, MNRAS, 339,105

Muller, E., Staveley-Smith, L., \& Zealey, W. J. 2003b, MNRAS, 338, 609

Nakajima, Y., Kato, D., Nagata, T., et al. 2005, AJ, 129, 776

Nishiyama, S., Haba, Y., Kato, D., et al. 2007, ApJ, 658, 358

Noël, N. E. D., Conn, B. C., Carrera, R., et al. 2013, ApJ, 768, 109

Onodera, S., Kuno, N., Tosaki, T., et al. 2010, ApJL, 722, L127

Parker, Q. A., Phillipps, S., Pierce, M. J., et al. 2005, MNRAS, 362, 689

Pineda, J. L., Goldsmith, P. F., Chapman, N., et al. 2010, ApJ, 721, 686

Poglitsch, A., Krabbe, A., Madden, S. C., et al. 1995, ApJ, 454, 293

Pomarès, M., Zavagno, A., Deharveng, L., et al. 2009, A\&A, 494, 987

Pottasch, S. R. 1993, in Infrared Astronomy, ed. A. Mampaso, M. Prieto, \& F. Sanchez (Cambridge: Cambridge Univ. Press), 63

Robitaille, T. P., Whitney, B. A., Indebetouw, R., \& Wood, K. 2007, ApJS, 169,328

Robitaille, T. P., Whitney, B. A., Indebetouw, R., Wood, K., \& Denzmore, P. 2006, ApJS, 167, 256

Rolleston, W. R. J., Dufton, P. L., McErlean, N. D., \& Venn, K. A. 1999, A\&A, 348,728

Rowan-Robinson, M., Babbedge, T., Surace, J., et al. 2005, AJ, 129, 1183

Saunders, W., Sutherland, W. J., Maddox, S. J., et al. 2000, MNRAS, 317, 55

Schruba, A., Leroy, A. K., Walter, F., Sandstrom, K., \& Rosolowsky, E. 2010, ApJ, 722, 1699

Seale, J. P., Looney, L. W., Chu, Y.-H., et al. 2009, ApJ, 699, 150

Sewiło, M., Carlson, L. R., Seale, J. P., et al. 2013, ApJ, 778, 15

Skrutskie, M. F., Cutri, R. M., Stiening, R., et al. 2006, AJ, 131, 1163

Stanimirovic, S., Staveley-Smith, L., Dickey, J. M., Sault, R. J., \& Snowden, S. L. 1999, MNRAS, 302, 417

Stetson, P. B. 1987, PASP, 99, 191

Struck, C. 1997, ApJS, 113, 269

Tabur, V., Bedding, T. R., Kiss, L. L., et al. 2010, MNRAS, 409, 777

Vaidya, K., Chu, Y., Gruendl, R. A., Chen, C., \& Looney, L. W. 2009, ApJ, 707, 1417

Whitney, B. A., Indebetouw, R., Babler, B. L., et al. 2004a, ApJS, 154, 315

Whitney, B. A., Indebetouw, R., Bjorkman, J. E., \& Wood, K. 2004b, ApJ, 617,1177

Whitney, B. A., Sewilo, M., Indebetouw, R., et al. 2008, AJ, 136, 18

Wong, T., Hughes, A., Fukui, Y., et al. 2009, ApJ, 696, 370 